\title{
Effective Local Search Algorithms for Routing and Scheduling Problems with General Time Window Constraints
}

\author{
T. Ibaraki, ${ }^{\dagger}$ S. Imahori, ${ }^{\dagger}$ M. Kubo,$^{\ddagger}$ T. Masuda, ${ }^{b}$ T. Uno ${ }^{\natural}$ and M. Yagiura ${ }^{\dagger *}$ \\ ${ }^{\dagger}$ Department of Applied Mathematics and Physics, Graduate School of Informatics, Kyoto University, \\ Kyoto 606-8501, Japan, Phone/Fax: +81-75-753-5494, E-mail: \{ibaraki,yagiura\}@i.kyoto-u.ac.jp, \\ imahori@amp.i.kyoto-u.ac.jp \\ ${ }^{\ddagger}$ Logistics and Information Engineering, Tokyo University of Mercantile Marine, Tokyo 135-8533, Japan, \\ E-mail: kubo@ipc.tosho-u.ac.jp \\ ${ }^{b}$ Communication \& High Technology, Accenture, Nihon Seimei Akasaka Daini Bldg., 7-1-16 Akasaka Minato- \\ ku, Tokyo 107-8672, Japan, E-mail: tomoyasu.masuda@accenture.com \\ ${ }^{\natural}$ National Institute of Informatics, Algorithm Foundation Research, National Center of Sciences, 2-1-2 \\ Hitotsubashi, Chiyoda-ku, Tokyo 101-8430, Japan, E-mail: uno@nii.ac.jp \\ * corresponding author
}

\begin{abstract}
We propose local search algorithms for the vehicle routing problem with soft time window constraints. The time window constraint for each customer is treated as a penalty function, which is very general in the sense that it can be non-convex and discontinuous as long as it is piecewise linear. In our algorithm, we use local search to assign customers to vehicles and to find orders of customers for vehicles to visit. It employs an advanced neighborhood, called the cyclic exchange neighborhood, in addition to standard neighborhoods for the vehicle routing problem. After fixing the order of customers for a vehicle to visit, we must determine the optimal start times of processing at customers so that the total penalty is minimized. We show that this problem can be efficiently solved by using dynamic programming, which is then incorporated in our algorithm. We then report computational results for various benchmark instances of the vehicle routing problem. The generality of time window constraints allows us to handle a wide variety of scheduling problems. As such an example, we mention in this paper an application to a production scheduling problem with inventory cost, and report computational results for real world instances.
\end{abstract}

Keywords: adaptive multi-start local search, dynamic programming, general time window constraints, local search, metaheuristics, vehicle routing problem, very large scale neighborhood.

\section{Introduction}

The vehicle routing problem (VRP) is the problem of minimizing the total distance traveled by a number of vehicles, under various constraints, where each customer must be visited exactly once by a vehicle (Desrochers et al. 1988, Desrosiers et al. 1995, Solomon and Desrosiers 1988). This is one of the representative combinatorial optimization problems and is known to be NP- 
hard. Among variants of VRP, the VRP with capacity and time window constraints, called the vehicle routing problem with time windows (VRPTW), has been widely studied in the last decade (Koskosidis, Powell and Solomon 1992, Potvin et al. 1996, Savelsbergh 1992, Taillard et al. 1997). The capacity constraint signifies that the total load on a route cannot exceed the capacity of the vehicle serving the route. The time window constraint signifies that each vehicle must start the service at each customer in the period specified by the customer. The VRPTW has a wide range of applications such as bank deliveries, postal deliveries, school bus routing and so on, and it has been a subject of intensive research focused mainly on heuristic and metaheuristic approaches (Bräysy and Gendreau 2003a, 2003b). Among recent approaches are a two-stage hybrid local search by Bent and Van Hentenryck (2001), a hybrid genetic algorithm by Berger, Barkaoui and Bräysy (2003), a reactive variable neighborhood search by Bräysy (2003), a two-phase hybrid metaheuristic algorithm by Homberger and Gehring (2003), a variable neighborhood decent with constraint-based operators by Rousseau, Gendreau and Pesant (2002) and so on. See an extensive survey by Bräysy and Gendreau (2003a, 2003b) for heuristic and metaheuristic approaches. To the best of our knowledge, only a convex time window constraint is allowed for each customer in most of the previous work of VRPTW (Potvin et al. 1996, Solomon 1987, Taillard et al. 1997). The case with multiple time windows (De Jong, Kant and Van Vliet 1996) and other variants of VRPTW (Solomon and Desrosiers 1988) are also considered to be important.

A constraint is called hard if it must be satisfied and is called soft if it can be violated. The amount of violation of soft constraints is usually penalized and added to the objective function. The VRP with hard (resp., soft) time window constraints is abbreviated as VRPHTW (resp., VRPSTW). For VRPHTW, even just finding a feasible schedule with a given number of vehicles is known to be NP-complete, because it includes the one-dimensional bin packing problem as a special case (Garey and Johnson 1979). Therefore, searching within the feasible region of VRPHTW may be inefficient, especially when the constraints are tight. Moreover, in many real-world situations, constraints of time window and capacity can be violated to some extent. Considering these, we treat these two constraints as soft in this paper.

The main contribution of this paper is to propose an efficient algorithm to deal with general time window constraints, which is then incorporated in metaheuristic algorithms to develop general problem solvers. The time window constraints we consider in this paper are general in the sense that one or more time slots can be assigned to each customer. That is, the corresponding penalty function can be non-convex and discontinuous as long as it is piecewise linear. We call the resulting problem as the vehicle routing problem with general time windows (VRPGTW). In this case, after fixing the order of customers for a vehicle to visit, we must determine the optimal start times of services at all customers so that the total time penalty of the vehicle is 
minimized. We show that this problem can be efficiently solved by using dynamic programming.

Let $n_{k}$ be the number of customers assigned to vehicle $k$, and $\delta_{k}$ be the total number of linear pieces of the penalty functions for those customers. The information of each linear piece is usually explicitly given, and in this case, $\delta_{k}$ is considered as the input size of the penalty functions of the customers. Moreover, $\delta_{k}=O\left(n_{k}\right)$ holds in many cases, since the number of linear pieces of the penalty function for each customer is usually $O(1)$. For example, if the penalty for a customer is the weighted sum of earliness and tardiness, then the number of linear pieces of the penalty function is at most 3. The time complexity of our dynamic programming is $O\left(n_{k} \delta_{k}\right)$ if the problem for vehicle $k$ is solved from scratch. We also show that the optimal time penalty of each solution in the neighborhood of the current solution can be evaluated in $O\left(\sum_{k \in M^{\prime}} \delta_{k}\right)$ time from the information of the current solution, where $M^{\prime}$ is the set of indices of vehicles which the neighborhood operation involves.

Special cases of convex penalty functions were considered in the literature of VRPSTW and scheduling problems, e.g., Davis and Kanet (1993), Garey, Tarjan and Wilfong (1988), Koskosidis, Powell and Solomon (1992), Taillard et al. (1997), Tamaki, Komori and Abe (1999), and Tamaki, Sugimoto and Araki (1999). In Taillard et al. (1997), the time penalty for each customer is $+\infty$ for earliness and linear for tardiness, and an $O(1)$ time algorithm to approximately compute the optimal time penalty of a solution in the neighborhood was proposed. In Davis and Kanet (1993), Koskosidis, Powell and Solomon (1992), Tamaki, Komori and Abe (1999), Tamaki, Sugimoto and Araki (1999), the time penalty is linear for both of earliness and tardiness, and an $O\left(n_{k}^{2}\right)$ time algorithm for a given route of vehicle $k$ was proposed in Davis and Kanet (1993) and Tamaki, Sugimoto and Araki (1999). If the penalty function for each customer is the absolute deviation from a specified time, this problem becomes the isotonic median regression problem, which has been extensively studied. To our knowledge, the best time complexity for this problem (for a vehicle $k$ ) is $O\left(n_{k} \log n_{k}\right.$ ) (Ahuja and Orlin 2001, Garey, Tarjan and Wilfong 1988, Hochbaum and Queyranne 2003). Very fast algorithms for general convex functions are also known (Ahuja and Orlin 2001, Hochbaum and Queyranne 2003).

The essential part of VRPGTW, i.e., assigning customers to vehicles and determining the visiting order of each vehicle, is solved by local search (LS) algorithms. In the literature, three types of neighborhoods, called the cross exchange, 2-opt* and Or-opt neighborhoods, have been widely used (Or 1976, Potvin et al. 1996, Reiter and Sherman 1965, Taillard et al. 1997). We refer to these neighborhoods as standard neighborhoods. In our local search, in addition to these standard neighborhoods, we use an advanced neighborhood called the cyclic exchange neighborhood (Ahuja et al. 2002, Ahuja, Orlin and Sharma 2000). This is defined to be the set of solutions obtainable by cyclically exchanging two or more paths of length at most $L^{\text {cyclic }}$ (a parameter), which stems from the cyclic transfer algorithm of Thompson and Psaraftis (1993) 
and the ejection chains of Glover (1996). As the size of this neighborhood grows exponentially with the input size, we search an improving solution by using the improvement graph, whose concept is proposed, e.g., in Ahuja et al. (2002) and Ahuja, Orlin and Sharma (2000), and is applicable to wide range of problems. We also propose time-oriented neighbor-lists to make the search in the cross exchange and 2-opt* neighborhoods more efficient.

Among many possible metaheuristics based on local search, we use the multi-start local search (MLS) (Lin 1965, Lin and Kernighan 1973, Reiter and Sherman 1965), the iterated local search (ILS) (Johnson 1990, Martin, Otto and Felten 1991, Martin, Otto and Felten 1992) and the adaptive multi-start local search (AMLS) (Boese, Kahng and Muddu 1994, Taillard et al. 1997). MLS repeatedly applies LS to a number of initial solutions which are generated randomly or by greedy methods, and the best solution obtained in the entire search is output. ILS is a variant of MLS, in which the initial solutions for LS are generated by perturbing good solutions obtained in the previous search. AMLS is also a variant of MLS, which keeps a set $P$ of good solutions found in the previous search, and generates initial solutions by combining the parts of the solutions in $P$.

We conduct computational experiments on three different types of instances: (1) Solomon's VRPHTW benchmark instances (Solomon 1987), (2) artificially generated instances of the parallel machine scheduling problem with various types of time windows, and (3) real world instances of a production scheduling problem with inventory cost. The computational results exhibit a good prospect of ILS and AMLS. For Solomon's benchmark instances, the solution quality of our algorithms is competitive with those of recent algorithms developed for VRPHTW, though our algorithms require slightly more computational time. It should be pointed out that Solomon's instances have only convex penalty functions, while our algorithms can handle more general penalty functions. For the parallel machine scheduling problem, we generate instances with various types of time penalty functions (including non-convex ones), whose optimal solutions are known. Our algorithms find optimal or near optimal solutions with high probability. It is also observed that the cyclic exchange neighborhood is quite effective in dealing with non-convex time penalty functions. For the production scheduling problem with inventory cost, we use real world data provided by a company, and observe that the proposed algorithms find better schedules than those currently used in the company.

\section{Problem}

In this section, we formulate the vehicle routing problem with general time windows (VRPGTW). Let $G=(V, E)$ be a complete directed graph with a vertex set $V=\{0,1, \ldots, n\}$ and an edge set $E=\{(i, j) \mid i, j \in V, i \neq j\}$, and $M=\{1,2, \ldots, m\}$ be a set of vehicles. Vertex 0 is the depot 
and other vertices are customers. The following parameters are associated with each customer $i \in V$, each vehicle $k \in M$, and each edge $(i, j) \in E$ :

- a quantity $q_{i}(\geq 0)$ of goods to be delivered,

- a penalty function $p_{i}(t)(\geq 0)$ of the start time $t$ of the service,

- a service time $u_{i}(\geq 0)$,

- a capacity $Q_{k}(\geq 0)$,

- a distance $d_{i j}(\geq 0)$, and

- a travel time $t_{i j}(\geq 0)$.

We assume $q_{0}=0$ and $u_{0}=0$ for the depot 0 . Each vehicle can start from the depot after time 0 . Each penalty function $p_{i}(t)$ is nonnegative, piecewise linear and satisfies $p_{i}(t) \leq$ $\lim _{\varepsilon \rightarrow 0} \min \left\{p_{i}(t+\varepsilon), p_{i}(t-\varepsilon)\right\}$ at every discontinuous point $t$ (see Fig. 1 for example). This assumption is necessary to ensure the existence of an optimal solution. Note that $p_{i}(t)$ can be non-convex and discontinuous as long as it satisfies the above conditions. Distances $d_{i j}$ and travel times $t_{i j}$ are in general asymmetric; i.e., $d_{i j} \neq d_{j i}$ and $t_{i j} \neq t_{j i}$ may hold.

Let $\sigma_{k}$ denote the route traveled by vehicle $k$, where $\sigma_{k}(h)$ denote the $h$ th customer in $\sigma_{k}$, and let

$$
\boldsymbol{\sigma}=\left(\sigma_{1}, \sigma_{2}, \ldots, \sigma_{m}\right)
$$

Note that each customer $i$ is included in exactly one $\sigma_{k}$, and is visited by the vehicle exactly once.

We denote by $n_{k}$ the number of customers in $\sigma_{k}$ for $k \in M$. For convenience, we define $\sigma_{k}(0)=0$ and $\sigma_{k}\left(n_{k}+1\right)=0$ for all $k$ (i.e., each vehicle $k \in M$ leaves the depot and comes back to the depot). We also use a set of directed edges $\left\{\left(\sigma_{k}(h), \sigma_{k}(h+1)\right) \in E \mid h=0,1, \ldots, n_{k}, k \in M\right\}$ to represent a set of routes $\boldsymbol{\sigma}$. Moreover, let $s_{i}$ be the start time of the service at customer $i$ and $s_{k}^{\text {a }}$ be the arrival time of vehicle $k$ at the depot, and let

$$
\boldsymbol{s}=\left(s_{1}, s_{2}, \ldots, s_{n}, s_{1}^{\mathrm{a}}, s_{2}^{\mathrm{a}}, \ldots, s_{m}^{\mathrm{a}}\right) .
$$

Note that each vehicle is allowed to wait at customers before starting services.

For convenience, we define $0-1$ variables $y_{i k}(\boldsymbol{\sigma}) \in\{0,1\}$ for $i \in V$ and $k \in M$ by

$$
y_{i k}(\boldsymbol{\sigma})=1 \Longleftrightarrow i=\sigma_{k}(h) \text { holds for exactly one } h \in\left\{1,2, \ldots, n_{k}\right\} \text {. }
$$

That is, $y_{i k}(\boldsymbol{\sigma})=1$ if and only if vehicle $k$ visits customer $i$. Then the total distance $d_{\text {sum }}(\boldsymbol{\sigma})$ traveled by all vehicles, the total penalty $p_{\text {sum }}(s)$ for start times of services, and the total amount $q_{\text {sum }}(\boldsymbol{\sigma})$ of capacity excess are expressed as

$$
\begin{aligned}
d_{\text {sum }}(\boldsymbol{\sigma}) & =\sum_{k \in M} \sum_{h=0}^{n_{k}} d_{\sigma_{k}(h), \sigma_{k}(h+1)} \\
p_{\text {sum }}(\boldsymbol{s}) & =\sum_{i \in V \backslash\{0\}} p_{i}\left(s_{i}\right)+\sum_{k \in M} p_{0}\left(s_{k}^{\mathrm{a}}\right)
\end{aligned}
$$




$$
q_{\mathrm{sum}}(\boldsymbol{\sigma})=\sum_{k \in M} \max \left\{\sum_{i \in V} q_{i} y_{i k}(\boldsymbol{\sigma})-Q_{k}, 0\right\} .
$$

The VRPGTW is now formulated as follows:

$$
\begin{aligned}
& \text { minimize } \quad \operatorname{cost}(\boldsymbol{\sigma}, \boldsymbol{s})=d_{\mathrm{sum}}(\boldsymbol{\sigma})+p_{\mathrm{sum}}(\boldsymbol{s})+q_{\mathrm{sum}}(\boldsymbol{\sigma}) \\
& \text { subject to } \quad \sum_{k \in M} y_{i k}(\boldsymbol{\sigma})=1, \quad i \in V \backslash\{0\} \\
& t_{0, \sigma_{k}(1)} \leq s_{\sigma_{k}(1)}, \quad k \in M \\
& s_{\sigma_{k}(h)}+u_{\sigma_{k}(h)}+t_{\sigma_{k}(h), \sigma_{k}(h+1)} \leq s_{\sigma_{k}(h+1)}, h=1,2, \ldots, n_{k}-1, k \in M \\
& s_{\sigma_{k}\left(n_{k}\right)}+u_{\sigma_{k}\left(n_{k}\right)}+t_{\sigma_{k}\left(n_{k}\right), 0} \leq s_{k}^{\mathrm{a}}, \quad k \in M \text {. }
\end{aligned}
$$

Constraint (2) means that every customer $i \in V \backslash\{0\}$ must be served only once by exactly one vehicle. Constraints (3), (4) and (5) require that the start time $s_{i}$ of the service at customer $i$ must be after the arrival time at customer $i$. The time window and capacity constraints are treated as soft, and their violation is evaluated as the penalties $p_{\text {sum }}(\boldsymbol{s})$ and $q_{\text {sum }}(\boldsymbol{\sigma})$ in the objective function.

As for the objective function (1), the weighted $\operatorname{sum} d_{\text {sum }}(\boldsymbol{\sigma})+\alpha p_{\text {sum }}(\boldsymbol{s})+\beta q_{\text {sum }}(\boldsymbol{\sigma})$ with constants $\alpha(\geq 0)$ and $\beta(\geq 0)$ might seem more natural; however, such weights can be treated in the above formulation by regarding $\alpha p_{i}(t), \beta q_{i}$ and $\beta Q_{k}(i \in V, k \in M)$ as the given data, and hence the weights are omitted for simplicity.

We can also consider a penalty function $\tilde{p}_{k}(t)$ of the departure time $t$ of vehicle $k$ from the depot, though in the above formulation we assume that all vehicles have the same departure time 0 . For this, we introduce $m$ dummy customers $i=n+1, n+2, \ldots, n+m$ with penalty functions $p_{i}(t)=\tilde{p}_{i-n}(t)$ to represent the penalty of the departure time of vehicle $k$ from the depot. Finally, the distances $d_{i j}$ and the travel times $t_{i j}$ are defined so that each vehicle $k$ must visit customer $n+k$ first (i.e., very large cost is incurred if vehicle $k$ visits other customers first).

The time window constraint of customer $i$ is often regarded as hard and defined by an interval $\left[w_{i}^{\mathrm{r}}, w_{i}^{\mathrm{d}}\right]$ (instead of a penalty function $p_{i}(t)$ ), within which the service of $i$ must be started. Such an instance can be treated as a special case of our formulation by setting penalty functions as either

$$
p_{i}(t)=\alpha \cdot \max \left\{w_{i}^{\mathrm{r}}-t, 0, t-w_{i}^{\mathrm{d}}\right\}
$$

or

$$
p_{i}(t)= \begin{cases}0, & t \in\left[w_{i}^{\mathrm{r}}, w_{i}^{\mathrm{d}}\right] \\ \alpha, & \text { otherwise }\end{cases}
$$

where $\alpha$ is a large positive value.

To avoid confusion, we note throughout the paper (unless otherwise stated) that a feasible solution denotes a solution that satisfies constraints (2)-(5); i.e., a feasible solution (of our 
formulation) does not necessarily satisfy the time window and capacity constraints. Cases with hard time window and/or capacity constraints can be treated by setting the penalties $p_{\text {sum }}(s)$ and $q_{\text {sum }}(\boldsymbol{\sigma})$ sufficiently large. See Section 3.2 for more discussion about this matter.

Remark: The number of vehicles $m$ is a given constant in this paper, although it is sometimes treated as a decision variable in the literature. We adopt this assumption for the following two reasons. (1) In many applications, the number of vehicles $m$ (or the number of machines in scheduling problems) is fixed. (2) Algorithms become simpler if $m$ is treated as a constant, since route elimination operators are not necessary. For problems where $m$ is a decision variable, we need to try various values of $m$ to find a small feasible $m$; however, in many practical situations, an appropriate range of $m$ is known in advance.

\section{Optimal start time of services}

In this section, we consider the problem of determining the time to start services of customers in a given route $\sigma_{k}$ so that the total time penalty is minimized. How to determine $\sigma_{k}$ will be discussed in Section 3. Let $\delta^{(i)}$ be the number of pieces in the piecewise linear function $p_{i}(t)$, and let the total number of pieces in the penalty functions for all the customers in $\sigma_{k}$ (including the depot) be $\delta_{k}=\sum_{h=0}^{n_{k}} \delta^{\left(\sigma_{k}(h)\right)}$. Furthermore, let $\delta=\sum_{k \in M} \delta_{k}=\sum_{i \in V \backslash\{0\}} \delta^{(i)}+m \delta^{(0)}$ be the total number of pieces in the penalty functions of all customers (including the depot), where $\delta^{(0)}$ is multiplied by $m$.

We propose an $O\left(n_{k} \delta_{k}\right)$ time algorithm based on the dynamic programming (DP) to solve this problem. (It is pointed out by Hochbaum (2002b) that this time complexity can also be achieved by using a more general algorithm in Hochbaum (1997), Hochbaum (2002a) and Hochbaum and Naor (1994); however, our DP algorithm is simpler and is used to achieve a more efficient algorithm in Section 4.1.2.) If the penalty functions are convex, this problem can be formulated as a convex programming problem, which is efficiently solvable by using existing methods (Bertsekas 1995, Chvátal 1983) even without using efficient algorithms for this problem (Ahuja and Orlin 2001, Hochbaum and Queyranne 2003). Here, it is emphasized that the proposed DP algorithm is applicable even if $p_{i}(t)$ are non-convex and discontinuous.

\subsection{The dynamic programming algorithm}

We define $f_{h}^{k}(t)$ to be the minimum sum of the penalty values for customers $\sigma_{k}(0), \sigma_{k}(1), \ldots, \sigma_{k}(h)$ under the condition that all of them are served before time $t$. Throughout this paper, we call this a forward minimum penalty function. For convenience, we also define

$$
\begin{aligned}
p_{h}^{k}(t) & =p_{\sigma_{k}(h)}(t), \\
\tau_{h}^{k} & =u_{\sigma_{k}(h)}+t_{\sigma_{k}(h), \sigma_{k}(h+1)},
\end{aligned}
$$


where $p_{h}^{k}(t)$ is the penalty function for the $h$ th customer of vehicle $k$, and $\tau_{h}^{k}$ is the sum of the service time at the $h$ th customer and the travel time from the $h$ th customer to the $(h+1)$ st customer. Then $f_{h}^{k}(t)$ can be computed by

$$
\begin{aligned}
& f_{0}^{k}(t)= \begin{cases}+\infty, & t \in(-\infty, 0) \\
0, & t \in[0,+\infty)\end{cases} \\
& f_{h}^{k}(t)=\min _{t^{\prime} \leq t}\left(f_{h-1}^{k}\left(t^{\prime}-\tau_{h-1}^{k}\right)+p_{h}^{k}\left(t^{\prime}\right)\right), \quad 1 \leq h \leq n_{k}+1,-\infty<t<+\infty .
\end{aligned}
$$

Then the minimum penalty value for the route $\sigma_{k}$ can be obtained by

$$
\min _{t} f_{n_{k}+1}^{k}(t)
$$

The optimal start time $s_{\sigma_{k}(h)}$ of the service for each $h=1,2, \ldots, n_{k}$ and the time $s_{k}^{\text {a }}$ of vehicle $k$ to return to the depot can be computed by

$$
\begin{aligned}
& s_{k}^{\mathrm{a}}=\min \arg \min _{t} f_{n_{k}+1}^{k}(t) \\
& s_{\sigma_{k}(h)}=\min \arg \min _{t \leq s_{\sigma_{k}(h+1)}-\tau_{h}^{k}} f_{h}^{k}(t), \quad 1 \leq h \leq n_{k},
\end{aligned}
$$

where $s_{0}=s_{k}^{\mathrm{a}}$ is assumed for convenience. The first min in the right hand side of (10) signifies the leftmost $t$ if $\min f_{n_{k}+1}^{k}(t)$ (or $\min f_{h}^{k}(t)$ ) is achieved by multiple $t$.

An example of the computation of recursion (8) is shown in Fig. 2. This figure represents the computation of $f_{h}^{k}(t)$ from $f_{h-1}^{k}(t)$ and $p_{h}^{k}(t)$, where $\tau_{h-1}^{k}=2$, and $f_{h-1}^{k}(t)$ and $p_{h}^{k}(t)$ are respectively defined by

$$
f_{h-1}^{k}(t)= \begin{cases}-t+3, & t<1 \\ 2, & 1 \leq t<4 \\ -t+6, & 4 \leq t<5 \\ 1, & 5<t\end{cases}
$$

and

$$
p_{h}^{k}(t)= \begin{cases}-0.5 t+3, & t<4 \\ t-3, & 4 \leq t<8 \\ -4 t+37, & 8 \leq t<9 \\ t-8, & 9<t .\end{cases}
$$

\subsection{Implementation and time complexity of the algorithm}

Let us consider the data structure for computing recursion (8). Since penalty function $p_{h}^{k}(t)$ is piecewise linear, $f_{h}^{k}(t)$ is also piecewise linear. Therefore we can keep the functions that appear in (8) in linked lists, whose components store the intervals and the associated linear functions (i.e., linear pieces) of the piecewise linear functions. In computing $f_{h}^{k}(t)$, the intervals of $f_{h-1}^{k}(t)$ are first shifted by $\tau_{h-1}^{k}$ to the right to obtain $f_{h-1}^{k}\left(t-\tau_{h-1}^{k}\right)$. Then, $f_{h-1}^{k}\left(t-\tau_{h-1}^{k}\right)+p_{h}^{k}(t)$ is 
computed by merging the intervals of $f_{h-1}^{k}\left(t-\tau_{h-1}^{k}\right)$ and $p_{h}^{k}(t)$ while adding the linear functions of the corresponding pieces, and by storing $f_{h-1}^{k}\left(t-\tau_{h-1}^{k}\right)+p_{h}^{k}(t)$ in a new linked list. Finally, $f_{h}^{k}(t)$ is obtained by taking the minimum of $f_{h-1}^{k}\left(t^{\prime}-\tau_{h-1}^{k}\right)+p_{h}^{k}\left(t^{\prime}\right)$ over all $t^{\prime} \leq t$, which can be achieved by scanning the new list from the left. Fig. 3 shows the linked lists for the functions in Fig. 2.

The computation of $f_{h-1}^{k}\left(t-\tau_{h-1}\right)+p_{h}^{k}(t)$ and $f_{h}^{k}(t)$ from $f_{h-1}^{k}(t)$ and $p_{h}^{k}(t)$ can be achieved in $O\left(\delta_{k}\right)$ time, since the total number of pieces in $f_{h-1}^{k}(t)$ and $p_{h}^{k}(t)$ is $O\left(\delta_{k}\right)$. The computation of total time penalty (9) and optimal start time (10) for each $h$ can also be achieved in $O\left(\delta_{k}\right)$ time, since both computations only require to scan the list of $f_{h}^{k}(t)$. Therefore, determining the minimum time penalty for a given route and the optimal start times of services for all the customers in the route can be done in $O\left(n_{k} \delta_{k}\right)$ time. (For evaluating the optimal time penalty of a solution in the neighborhood, a more efficient algorithm will be explained in Section 4.1.2.)

\section{Local search}

In this section, we describe the framework of our local search (LS). The search space of LS is the set of all visiting orders $\boldsymbol{\sigma}=\left(\sigma_{1}, \sigma_{2}, \ldots, \sigma_{m}\right)$ satisfying condition (2). Then a solution $\boldsymbol{\sigma}$ is evaluated by

$$
\operatorname{cost}(\boldsymbol{\sigma})=d_{\mathrm{sum}}(\boldsymbol{\sigma})+p_{\mathrm{sum}}^{*}(\boldsymbol{\sigma})+q_{\mathrm{sum}}(\boldsymbol{\sigma}),
$$

where $p_{\text {sum }}^{*}(\boldsymbol{\sigma})$ is the minimum value of $p_{\text {sum }}(\boldsymbol{s})$ among those $\boldsymbol{s}$ satisfying conditions (3)-(5) for the given $\boldsymbol{\sigma}$. Such an $s$ is computed by the dynamic programming of Section 2 (a more efficient method will be explained in Section 4.1.2). For convenience, let $d_{\text {sum }}\left(\sigma_{k}\right)$ be the total distance of route $\sigma_{k}, p_{\mathrm{sum}}^{*}\left(\sigma_{k}\right)$ be the optimal time penalty of route $\sigma_{k}$, and $q_{\mathrm{sum}}\left(\sigma_{k}\right)$ be the total amount of capacity excess of route $\sigma_{k}$, and define $\operatorname{cost}\left(\sigma_{k}\right)=d_{\mathrm{sum}}\left(\sigma_{k}\right)+p_{\mathrm{sum}}^{*}\left(\sigma_{k}\right)+q_{\mathrm{sum}}\left(\sigma_{k}\right)$. Then $\operatorname{cost}(\boldsymbol{\sigma})=\sum_{k \in M} \operatorname{cost}\left(\sigma_{k}\right)$ holds.

The neighborhood $N(\boldsymbol{\sigma})$ of a feasible solution $\boldsymbol{\sigma}$ is a set of solutions obtainable from $\boldsymbol{\sigma}$ by applying some specified operations (to be described later). The LS starts from an initial solution $\boldsymbol{\sigma}$ and repeats replacing $\boldsymbol{\sigma}$ with a better solution $\boldsymbol{\sigma}^{\prime}$ (i.e., $\operatorname{cost}\left(\boldsymbol{\sigma}^{\prime}\right)<\operatorname{cost}(\boldsymbol{\sigma})$ ) in its neighborhood $N(\boldsymbol{\sigma})$ until no better solution is found in $N(\boldsymbol{\sigma})$. We will use more than one neighborhood in our LS.

In the subsequent sections, we will explain the details of LS. In Section 3.1, we explain the neighborhoods used in our algorithm. In Section 3.2, we explain how we define the incumbent solution. In Section 3.3, we describe the search order in the neighborhoods, and summarize the framework of LS. 


\subsection{Neighborhoods}

In our algorithm, we use the standard neighborhoods (i.e., the cross exchange, 2-opt* and Or-opt neighborhoods) with slight modifications. We also use the cyclic exchange neighborhood, whose basic idea was proposed in Ahuja et al. (2002) and Ahuja, Orlin and Sharma (2000).

\subsubsection{Standard neighborhoods}

The cross exchange neighborhood was proposed in Taillard et al. (1997). A cross exchange operation removes two paths from two different routes (one from each), whose length (i.e., the number of customers in the path) is at most $L^{\text {cross }}$ (a parameter), and exchanges them. Let $N^{\text {cross }}\left(\boldsymbol{\sigma}, k, k^{\prime}\right)$ be the set of all solutions obtainable by cross exchange operations on two routes $\sigma_{k}$ and $\sigma_{k^{\prime}}$ in the current solution $\boldsymbol{\sigma}=\left(\sigma_{1}, \sigma_{2}, \ldots, \sigma_{m}\right)$, and let $N^{\text {cross }}(\boldsymbol{\sigma})=\bigcup_{k<k^{\prime}} N^{\text {cross }}\left(\boldsymbol{\sigma}, k, k^{\prime}\right)$. The size of the cross exchange neighborhood is $O\left(n^{2}\left(L^{\text {cross }}\right)^{2}\right)$.

Fig. 4 illustrates a cross exchange operation. In this figure, squares represent the depot (which is duplicated at each end) and circles represent customers in the route. A thin line represents a route edge and a thick line represents a path (i.e., more than two customers may be included). First, two edges $\left(\sigma_{k}\left(h_{1}^{k}-1\right), \sigma_{k}\left(h_{1}^{k}\right)\right)$ and $\left(\sigma_{k}\left(h_{2}^{k}-1\right), \sigma_{k}\left(h_{2}^{k}\right)\right)$ are removed from route $\sigma_{k}$ and two edges $\left(\sigma_{k^{\prime}}\left(h_{1}^{k^{\prime}}-1\right), \sigma_{k^{\prime}}\left(h_{1}^{k^{\prime}}\right)\right)$ and $\left(\sigma_{k^{\prime}}\left(h_{2}^{k^{\prime}}-1\right), \sigma_{k^{\prime}}\left(h_{2}^{k^{\prime}}\right)\right)$ are also removed from route $\sigma_{k^{\prime}}$. Then, four new edges $\left(\sigma_{k}\left(h_{1}^{k}-1\right), \sigma_{k^{\prime}}\left(h_{1}^{k^{\prime}}\right)\right),\left(\sigma_{k^{\prime}}\left(h_{2}^{k^{\prime}}-1\right), \sigma_{k}\left(h_{2}^{k}\right)\right),\left(\sigma_{k^{\prime}}\left(h_{1}^{k^{\prime}}-1\right), \sigma_{k}\left(h_{1}^{k}\right)\right)$ and $\left(\sigma_{k}\left(h_{2}^{k}-1\right), \sigma_{k^{\prime}}\left(h_{2}^{k^{\prime}}\right)\right)$ are added to exchange two paths $\sigma_{k}\left(h_{1}^{k}\right) \rightarrow \sigma_{k}\left(h_{2}^{k}-1\right)$ and $\sigma_{k^{\prime}}\left(h_{1}^{k^{\prime}}\right) \rightarrow$ $\sigma_{k^{\prime}}\left(h_{2}^{k^{\prime}}-1\right)$.

The 2-opt* neighborhood was proposed in Potvin et al. (1996), which is a variant of the 2-opt neighborhood (Lin 1965) for the traveling salesman problem (TSP, a special case of VRP in which the number of vehicles is one). A 2-opt* operation removes two edges from two different routes (one from each) to divide each route into two parts and exchanges the second parts of the two routes (see Fig. 5). Let $N^{2 \mathrm{opt}^{*}}\left(\boldsymbol{\sigma}, k, k^{\prime}\right)$ be the set of all solutions obtainable by 2opt* operations on two routes $\sigma_{k}$ and $\sigma_{k^{\prime}}$ of the current solution $\boldsymbol{\sigma}=\left(\sigma_{1}, \sigma_{2}, \ldots, \sigma_{m}\right)$, and let $N^{2 \mathrm{opt}^{*}}(\boldsymbol{\sigma})=\bigcup_{k<k^{\prime}} N^{2 \mathrm{opt}^{*}}\left(\boldsymbol{\sigma}, k, k^{\prime}\right)$. The size of the 2-opt* neighborhood is $O\left(n^{2}\right)$.

Note that $N^{2 \text { opt }^{*}}(\boldsymbol{\sigma}) \subseteq N^{\text {cross }}(\boldsymbol{\sigma})$ holds if $L^{\text {cross }}=n$, since the cross exchange operation generates solutions in $N^{2 \mathrm{opt}^{*}}(\boldsymbol{\sigma})$ when the last customers of the two paths to be exchanged $\left(\sigma_{k}\left(h_{2}^{k}-1\right)\right.$ and $\sigma_{k^{\prime}}\left(h_{2}^{k^{\prime}}-1\right)$ in Fig. 4) are the last customers of the routes. However, parameter $L^{\text {cross }}$ is usually set small to keep $\left|N^{\text {cross }}(\boldsymbol{\sigma})\right|$ small and there are many solutions in $N^{2 \text { opt }^{*}}(\boldsymbol{\sigma}) \backslash$ $N^{\text {cross }}(\boldsymbol{\sigma})$. Hence searching in $N^{2 \operatorname{opt}^{*}}(\boldsymbol{\sigma})$ separately from $N^{\text {cross }}(\boldsymbol{\sigma})$ is meaningful.

The cross exchange and 2-opt* operations always change the assignment of customers to the routes. We therefore use the intra-route neighborhood to improve a solution within a route, which is a variant of Or-opt neighborhood used in TSP (Or 1976, Reiter and Sherman 1965). 
An intra-route operation removes a path of length at most $L_{\text {path }}^{\text {intra }}$ (a parameter) and inserts it into another position of the same route, where the position is limited within distance $L_{\text {ins }}^{\text {intra }}$ (a parameter) from the original position. This is illustrated in Fig. 6. If the removed path is $\sigma_{k}\left(h_{1}\right) \rightarrow \sigma_{k}\left(h_{1}+l-1\right)\left(1 \leq l \leq L_{\text {path }}^{\text {intra }}\right)$, it is inserted either between $\sigma_{k}\left(h_{1}-l^{\prime}-1\right)$ and $\sigma_{k}\left(h_{1}-l^{\prime}\right)$, or between $\sigma_{k}\left(h_{1}+l+l^{\prime}-1\right)$ and $\sigma_{k}\left(h_{1}+l+l^{\prime}\right)$, for $1 \leq l^{\prime} \leq L_{\text {ins }}^{\text {intra }}$. If the removed path is inserted between $\sigma_{k}\left(h_{1}+l+l^{\prime}-1\right)$ and $\sigma_{k}\left(h_{1}+l+l^{\prime}\right)$ (resp., between $\sigma_{k}\left(h_{1}-l^{\prime}-1\right)$ and $\sigma_{k}\left(h_{1}-l^{\prime}\right)$ ), it is called a forward (resp., backward) insertion. In Fig. 6, we show a forward insertion, where it is denoted $h_{2}=h_{1}+l+l^{\prime}-1$ for simplicity. For each insertion, we consider two cases: (1) with its visiting order preserved (denoted a normal insertion), and (2) with its visiting order reversed (denoted a reverse insertion). Note that, even if we only consider $l^{\prime} \geq 1$, the operation of just inverting the order of the removed path with its position unchanged is an intra-route operation using a forward reverse insertion with $l^{\prime}=1$ (i.e., the case with $h_{2}=h_{1}+l$ in Fig. $6(\mathrm{~b}))$, where the path $\sigma_{k}\left(h_{1}\right) \rightarrow \sigma_{k}\left(h_{1}+l\right)$ is regarded as the removed path.

Let $N^{\text {intra }}(\boldsymbol{\sigma}, k)$ be the set of all solutions obtainable by intra-route operations on route $\sigma_{k}$ of the current solution $\boldsymbol{\sigma}=\left(\sigma_{1}, \sigma_{2}, \ldots, \sigma_{m}\right)$, and let $N^{\text {intra }}(\boldsymbol{\sigma})=\bigcup_{k \in M} N^{\text {intra }}(\boldsymbol{\sigma}, k)$. The size of the intra-route neighborhood is $O\left(n L_{\text {path }}^{\text {intra }} L_{\text {ins }}^{\text {intra }}\right)$.

\subsubsection{The cyclic exchange neighborhood}

In this section, we define the cyclic exchange neighborhood. Let $\psi_{i l}$ denote the path in a solution $\boldsymbol{\sigma}$, whose initial customer is $i$ and whose length is $l$. That is, $\psi_{i l}$ denotes the sequence of $l$ consecutive customers $i=\sigma_{k}(h), \sigma_{k}(h+1), \ldots, \sigma_{k}(h+l-1)$. Let $k[i]$ denote the vehicle that visits customer $i$. For a sequence of paths $\psi_{i_{1} l_{1}}, \psi_{i_{2} l_{2}}, \ldots, \psi_{i_{r} l_{r}}$ satisfying $k\left[i_{j}\right] \neq k\left[i_{j^{\prime}}\right]$ for all $j \neq j^{\prime}$, the cyclic exchange operation is defined as follows: for each $j=1,2, \ldots, r, \psi_{i_{j-1}, l_{j-1}}$ is removed from route $\sigma_{k\left[i_{j-1}\right]}$ and inserted into route $\sigma_{k\left[i_{j}\right]}$ between the two customers to which $\psi_{i_{j} l_{j}}$ was connected $\left(i_{0}=i_{r}\right.$ and $l_{0}=l_{r}$ are assumed for convenience). Note that there are two directions in inserting a path: (1) with its visiting order unchanged (denoted normal insertion) and (2) with its visiting order reversed (denoted reverse insertion). As a result, there are $2^{r}$ possible cyclic exchange operations on a sequence of paths $\psi_{i_{1} l_{1}}, \psi_{i_{2} l_{2}}, \ldots, \psi_{i_{r} l_{r}}$. Fig. 7 represents an example of such cyclic exchange operations. In this figure, route $\sigma_{k\left[i_{1}\right]}$ is duplicated at the right end for simplicity.

As a special case of the cyclic exchange, we also consider the acyclic exchange operation. Let $\psi_{i 0}(i=1,2, \ldots, n)$ and $\psi_{00}^{k}(k=1,2, \ldots, m)$ denote empty paths (i.e., paths of length 0 starting from customer $i$ and the depot, respectively) in a solution $\boldsymbol{\sigma}$. An empty path means that no customer is removed from the route. Let $\psi_{i^{\prime} l^{\prime}}$ be the path to be inserted into the route from which $\psi_{i 0}$ or $\psi_{00}^{k}$ is removed. Then $\psi_{i 0}$ signifies that $\psi_{i^{\prime} l^{\prime}}$ is inserted between customer $i$ and its predecessor, while $\psi_{00}^{k}$ signifies that $\psi_{i^{\prime} l^{\prime}}$ is inserted between customer $\sigma_{k}\left(n_{k}\right)$ and the 
depot. Then the cyclic exchange operation on a sequence of paths $\psi_{i_{1} l_{1}}, \psi_{i_{2} l_{2}}, \ldots, \psi_{i_{r} l_{r}}$ is called the acyclic exchange, if an empty path is removed from route $k\left[i_{r}\right]$. Fig. 8 shows an acyclic exchange operation.

(Figure 8)

The cyclic exchange neighborhood $N^{\text {cyclic }}(\boldsymbol{\sigma})$ is defined to be the set of solutions obtainable by applying cyclic exchange operations (including acyclic exchange operations) on every possible combination of paths $\psi_{i l}$ satisfying the following three conditions:

1. all paths belong to different vehicles,

2. the length of each path is at most $L^{\text {cyclic }}$ (a parameter),

3. the number of participating paths is at most $\nu^{\text {cyclic }}$ (a parameter). If $L^{\text {cyclic }} \geq L^{\text {cross }}$ and $\nu^{\text {cyclic }} \geq 2$, then $N^{\text {cross }}(\boldsymbol{\sigma}) \subseteq N^{\text {cyclic }}(\boldsymbol{\sigma})$ holds.

The size of $N^{\text {cyclic }}(\boldsymbol{\sigma})$ is usually very large and grows exponentially with $\nu^{\text {cyclic }}$. Therefore, enumerating all solutions in $N^{\text {cyclic }}(\boldsymbol{\sigma})$ is computationally infeasible. However, the concept of the improvement graph (Ahuja et al. 2002, Ahuja, Orlin and Sharma 2000) can be utilized to implicitly search the neighborhood. We will describe the improvement graph and how we search an improved solution in Section 4.2 .

\subsection{The incumbent solution}

The incumbent solution in our algorithm is defined as follows. In our formulation of VRPGTW, the capacity and time window constraints are treated as soft. That is, they are not included in the constraints (2)-(5) of VRPGTW. Therefore, a locally optimal solution output by LS may not satisfy the two constraints (i.e., it may not be feasible to VRPHTW even though it is always feasible to VRPGTW). This situation may happen even if we set $\alpha$ in (6) or (7) very large, or multiply the values of $q_{i}$ and $Q_{k}$ by a very large number, to make $p_{\text {sum }}^{*}(\boldsymbol{\sigma})$ and $q_{\text {sum }}(\boldsymbol{\sigma})$ relatively large. On the other hand, the solutions found during the search of LS often satisfy the two constraints even if $p_{\text {sum }}^{*}(\boldsymbol{\sigma})$ and $q_{\text {sum }}(\boldsymbol{\sigma})$ are not set very large. Some applications prefer such solutions that satisfy the two constraints (i.e., VRPHTW). Thus, in addition to the original function $\operatorname{cost}(\boldsymbol{\sigma})$ of (11), we allow the algorithm to have another criterion, besteval( $\boldsymbol{\sigma})$, and to keep as the incumbent the feasible solution to VRPGTW (i.e., constraints (2)-(5) are satisfied), which has the smallest besteval $(\boldsymbol{\sigma})$ among those found during the search by then. For example, besteval may be $\operatorname{cost}(\boldsymbol{\sigma})$ or

$$
\operatorname{besteval}(\boldsymbol{\sigma})= \begin{cases}d_{\mathrm{sum}}(\boldsymbol{\sigma}), & \text { if } p_{\text {sum }}^{*}(\boldsymbol{\sigma})+q_{\mathrm{sum}}(\boldsymbol{\sigma})=0 \\ \operatorname{cost}(\boldsymbol{\sigma})+\beta, & \text { otherwise }\end{cases}
$$

where $\beta$ is an appropriate constant that satisfies $\beta>d_{\text {sum }}(\boldsymbol{\sigma})$ for all $\boldsymbol{\sigma}$. Here we emphasize that besteval does not affect the search process of our algorithm, i.e., the search is conducted entirely on the basis of cost. 


\subsection{The whole framework of the local search}

The LS in our algorithm searches the neighborhoods in the order described as follows. We first search the three neighborhoods,

1. the intra-route neighborhood,

2. the 2-opt* neighborhood, and

3. the cross exchange neighborhood,

in this order. The search of each neighborhood is executed until no improvement is found in the neighborhood. Then, after the cross exchange neighborhood, it returns to the intra-route neighborhood. This procedure continues until no improvement is found in three consecutive neighborhoods. The cyclic exchange neighborhood is then searched after this procedure. If an improved solution is found in the cyclic exchange neighborhood, the procedure immediately returns to the intra-route neighborhood; otherwise the local search outputs the locally optimal solution and stops. Our local search, $\operatorname{LS}\left(\boldsymbol{\sigma}^{0}\right)$, which starts from an initial solution $\boldsymbol{\sigma}^{0}$, is summarized as follows. (Note that we also keep the solution that minimizes besteval $(\boldsymbol{\sigma})$ of Section 3.2 among those solutions generated during the search process, though it is not explicitly described due to space limitation.)

\section{Algorithm LS $\left(\sigma^{0}\right)$}

Step 1 Let $\sigma:=\sigma_{0}$.

Step 2 Execute the following neighborhood search cyclically until no improvement is achieved in three consecutive neighborhoods.

2a (intra-route neighborhood) If there is a feasible solution $\boldsymbol{\sigma}^{\prime} \in N^{\text {intra }}(\boldsymbol{\sigma})$ such that $\operatorname{cost}\left(\boldsymbol{\sigma}^{\prime}\right)<\operatorname{cost}(\boldsymbol{\sigma})$, let $\boldsymbol{\sigma}:=\boldsymbol{\sigma}^{\prime}$, and return to Step 2a. Otherwise go to Step 2b.

$\mathbf{2 b}$ (2-opt* neighborhood) If there is a feasible solution $\boldsymbol{\sigma}^{\prime} \in N^{2 \mathrm{opt}^{*}}(\boldsymbol{\sigma}) \operatorname{such}$ that $\operatorname{cost}\left(\boldsymbol{\sigma}^{\prime}\right)<$ $\operatorname{cost}(\boldsymbol{\sigma})$, let $\boldsymbol{\sigma}:=\boldsymbol{\sigma}^{\prime}$, and return to Step 2b. Otherwise go to Step 2c.

2c (cross exchange neighborhood) If there is a feasible solution $\boldsymbol{\sigma}^{\prime} \in N^{\text {cross }}(\boldsymbol{\sigma})$ such that $\operatorname{cost}\left(\boldsymbol{\sigma}^{\prime}\right)\left\langle\operatorname{cost}(\boldsymbol{\sigma})\right.$, let $\boldsymbol{\sigma}:=\boldsymbol{\sigma}^{\prime}$, and return to Step 2c. Otherwise return to Step 2a.

Step 3 (cyclic exchange neighborhood) If there is a feasible solution $\boldsymbol{\sigma}^{\prime} \in N^{\text {cyclic }}(\boldsymbol{\sigma})$ such that $\operatorname{cost}\left(\boldsymbol{\sigma}^{\prime}\right)<\operatorname{cost}(\boldsymbol{\sigma})$, let $\boldsymbol{\sigma}:=\boldsymbol{\sigma}^{\prime}$ and return to Step 2. Otherwise output the current solution $\sigma$ and halt.

\section{Efficient implementation of local search}

In this section, we explain various ideas useful to search the neighborhood efficiently. In Section 4.1, we propose ideas to speed up the evaluation of the objective values of solutions in the standard neighborhoods. In Section 4.2, we explain the improvement graph and an algorithm to 
search in the cyclic exchange neighborhood. In Section 4.3, we propose time-oriented neighborlists to prune the search in the cross exchange and 2-opt* neighborhoods. Finally, in Section 4.4, we propose two more ideas to speed up the local search procedure.

\subsection{Evaluation of solutions in the standard neighborhoods}

Let $\Delta d_{\text {sum }}, \Delta p_{\text {sum }}$ and $\Delta q_{\text {sum }}$ be the differences in the distance $d_{\text {sum }}(\boldsymbol{\sigma})$, the time penalty $p_{\text {sum }}^{*}(\boldsymbol{\sigma})$, and the amount $q_{\text {sum }}(\boldsymbol{\sigma})$ of capacity excess, respectively, between the current solution and a solution in the neighborhood. Then let

$$
\Delta \text { cost }=\Delta d_{\text {sum }}+\Delta p_{\text {sum }}+\Delta q_{\text {sum }}
$$

which is negative if the new solution is better than the current solution. For a neighborhood $N$, let one-round time for $N$ be the time either (1) to find an improved solution in $N$ and update the data stored to evaluate solutions, or (2) to conclude that there is no improved solution in $N$. We also define one-round time for $N$ with respect to $d_{\text {sum }}$ to be the time required to evaluate $\Delta d_{\text {sum }}$ during the whole one-round time. One-round time for $N$ with respect to $p_{\text {sum }}$ and that with respect to $q_{\text {sum }}$ are similarly defined. In the subsequent sections, we explain separately how we evaluate $\Delta d_{\text {sum }}, \Delta p_{\text {sum }}$ and $\Delta q_{\text {sum }}$ during the search of cross exchange, 2-opt* ${ }^{*}$ and intra-route neighborhoods.

\subsubsection{Evaluation of $\Delta d_{\text {sum }}$}

We can compute $\Delta d_{\text {sum }}$ by the difference between the sum of distances of the inserted edges and that of the removed edges. For a solution in the cross exchange or 2-opt* neighborhood, $\Delta d_{\text {sum }}$ can be computed in $O(1)$ time, since the number of removed edges and inserted edges is constant. Therefore, one-round times for $N^{\text {cross }}$ and $N^{2 \text { opt }^{*}}$ with respect to $d_{\text {sum }}$ are $O\left(\left|N^{\text {cross }}\right|\right)$ and $O\left(\left|N^{20 p t^{*}}\right|\right)$, respectively.

For the intra-route neighborhood, the evaluation of $\Delta d_{\text {sum }}$ requires $O\left(L_{\text {path }}^{\text {intra }}\right)$ time if we evaluate a solution in $N^{\text {intra }}(\boldsymbol{\sigma})$ from scratch, since we need to compute the sum of the distances of all edges in the removed path when it is inserted reversely. However, we shall explain below that this computational time can be reduced to $O(1)$ and, consequently, one-round time for $N^{\text {intra }}$ with respect to $d_{\text {sum }}$ can be reduced from $O\left(\left|N^{\text {intra }}\right| \cdot L_{\text {path }}^{\text {intra }}\right)$ to $O\left(\left|N^{\text {intra }}\right|\right)$, if we evaluate the solutions in $N^{\text {intra }}$ in a specified order and use the information from the previous search.

As explained in Section 3.1.1, intra-route operation is categorized into the following four groups by the types of insertion: (1) normal forward insertion, (2) reverse forward insertion, (3) normal backward insertion, and (4) reverse backward insertion. Since $\Delta d_{\text {sum }}$ is easily computed in $O(1)$ time for normal insertions, and operations (2) and (4) are similar, we will only treat the case of (2). See Fig. 6 (b) for a help to understand the following discussion. 
The computation of reverse forward insertions on route $\sigma_{k}$ is done as shown in Fig. 9. The three nested loops in Fig. 9 generate all possible reverse forward insertions. The new routes $\sigma_{k}^{\text {new }}$ tested in this loop are $\left\langle\right.$ the depot $\rightarrow \sigma_{k}\left(h_{1}-1\right), \sigma_{k}\left(h_{1}+l\right) \rightarrow \sigma_{k}\left(h_{2}\right), \sigma_{k}\left(h_{1}+l-1\right) \rightarrow$ $\sigma_{k}\left(h_{1}\right), \sigma_{k}\left(h_{2}+1\right) \rightarrow$ the depot $\rangle$. To evaluate $\Delta d_{\text {sum }}$ in $O(1)$ time for each $\sigma_{k}^{\text {new }}$, we store two values $d i s t^{-}$and $d i s t^{+}$in the above nested loops. For simplicity, let $d_{h h^{\prime}}^{k}$ denote the distance from the $h$ th customer to the $h^{\prime}$ th customer in route $\sigma_{k}$. If $l=1$, we initialize dist ${ }^{-}$and $d i s t^{+}$ by $d i s t^{-}:=0$ and $d i s t^{+}:=0$; otherwise (i.e., $l>1$ ) we update them by

$$
\begin{aligned}
& \text { dist }^{-}:=\text {dist }^{-}+d_{h_{1}+l-2, h_{1}+l-1}^{k}, \\
& \text { dist }^{+}:=\text {dist }^{+}+d_{h_{1}+l-1, h_{1}+l-2}^{k} .
\end{aligned}
$$

The above initialization and update of $d i s t^{-}$and $d i s t^{+}$can be executed in $O(1)$ time. Then, $\Delta d_{\text {sum }}$ of $\sigma_{k}^{\text {new }}$ can be computed in $O(1)$ time by

$$
\begin{aligned}
\Delta d_{\text {sum }}= & \left(\text { dist }^{+}+d_{h_{1}-1, h_{1}+l}^{k}+d_{h_{2}, h_{1}+l-1}^{k}+d_{h_{1}, h_{2}+1}^{k}\right) \\
& -\left(d i s t^{-}+d_{h_{1}-1, h_{1}}^{k}+d_{h_{1}+l-1, h_{1}+l}^{k}+d_{h_{2}, h_{2}+1}^{k}\right) .
\end{aligned}
$$

Space complexity for this computation is $O(1)$.

\subsubsection{Evaluation of $\Delta p_{\text {sum }}$}

If $\Delta p_{\text {sum }}$ is computed by obtaining $p_{\text {sum }}^{*}$ of the new routes by (8) from scratch, it takes $O\left(\sum_{k \in M^{\prime}} n_{k} \delta_{k}\right)$ time, where $M^{\prime}$ is the set of indices of the routes related to the neighborhood operation $\left(\left|M^{\prime}\right| \leq 2\right.$ holds for standard neighborhoods). Instead of this, we propose an $O\left(\sum_{k \in M^{\prime}} \delta_{k}\right)=O(\delta)$ time algorithm that computes $\Delta p_{\text {sum. }}$. We also propose a simple idea to further reduce the computational time, though the worst case time complexity does not change.

Let us consider the computation of the minimum time penalty on a route $\sigma_{k}$. Define $b_{h}^{k}(t)$ to be the minimum sum of the penalty values for customers $\sigma_{k}(h), \sigma_{k}(h+1), \ldots, \sigma_{k}\left(n_{k}\right)$, $\sigma_{k}\left(n_{k}+1\right)$, provided that all of them are served after time $t$. We call this the backward minimum penalty function. Then, $b_{h}^{k}(t)$ can be computed as follows in a symmetric manner to the computation of $f_{h}^{k}(t)$,

$$
\begin{aligned}
b_{n_{k}+1}^{k}(t) & =\min _{t^{\prime} \geq t} p_{0}\left(t^{\prime}\right) \\
b_{h}^{k}(t) & =\min _{t^{\prime} \geq t}\left(b_{h+1}^{k}\left(t^{\prime}+\tau_{h}^{k}\right)+p_{h}^{k}\left(t^{\prime}\right)\right), \quad 1 \leq h \leq n_{k} .
\end{aligned}
$$

Then,

$$
p_{\mathrm{sum}}^{*}\left(\sigma_{k}\right)=\min _{t}\left(f_{h}^{k}(t)+b_{h+1}^{k}\left(t+\tau_{h}^{k}\right)\right)
$$

holds for any $h\left(1 \leq h \leq n_{k}\right)$. That is, if $f_{h}^{k}(t)$ and $b_{h+1}^{k}(t)$ are known for some $h$, the minimum penalty $p_{\text {sum }}^{*}\left(\sigma_{k}\right)$ can be computed by (13). This is possible in $O\left(\delta_{k}\right)$ time, because $f_{h}^{k}(t)$ and $b_{h+1}^{k}(t)$ consist of $O\left(\delta_{k}\right)$ linear pieces as explained in Section 2.2 (for the case of $f_{h}^{k}(t)$ ). 
To utilize this idea, we keep $f_{h}^{k}(t)$ and $b_{h}^{k}(t)$ in memory for all $h=1,2, \ldots, n_{k}$ and $k \in M$. In the case of neighborhood $N^{2 o p t}{ }^{*}$, the computation of (13) can be directly carried out by using the forward and backward minimum penalty functions stored in memory. In case of $N^{\text {cross }}$ and $N^{\text {intra }}$, however, the solutions must be treated in a specified order. We will describe the algorithm only for $N^{\text {intra }}$, since the case of $N^{\text {cross }}$ is simpler.

As in Section 4.1.1, we only consider the reverse forward insertions on a route $\sigma_{k}$ as other cases are similar. Recall that solutions are searched in the order as used in Fig. 9. Let $f_{h_{1}, h_{2}, l}(t)$ be the forward minimum penalty function for the subroute $\left\langle\right.$ the depot $\rightarrow \sigma_{k}\left(h_{1}-1\right), \sigma_{k}\left(h_{1}+l\right) \rightarrow$ $\left.\sigma_{k}\left(h_{2}\right)\right\rangle$, and $b_{h_{1}, h_{2}, l}(t)$ be the backward minimum penalty function for the subroute $\left\langle\sigma_{k}\left(h_{1}+\right.\right.$ $l-1) \rightarrow \sigma_{k}\left(h_{1}\right), \sigma_{k}\left(h_{2}+1\right) \rightarrow$ the depot $\rangle$ (see Fig. $6(\mathrm{~b})$ ). Then we evaluate the total time penalty of the new route $\sigma_{k}^{\text {new }}=\left\langle\right.$ the depot $\rightarrow \sigma_{k}\left(h_{1}-1\right), \sigma_{k}\left(h_{1}+l\right) \rightarrow \sigma_{k}\left(h_{2}\right), \sigma_{k}\left(h_{1}+l-1\right) \rightarrow$ $\sigma_{k}\left(h_{1}\right), \sigma_{k}\left(h_{2}+1\right) \rightarrow$ the depot $\rangle$ by

$$
\min _{t}\left(f_{h_{1}, h_{2}, l}(t)+b_{h_{1}, h_{2}, l}\left(t+u_{\sigma_{k}\left(h_{2}\right)}+t_{\sigma_{k}\left(h_{2}\right), \sigma_{k}\left(h_{1}+l-1\right)}\right)\right) .
$$

As stated above, this computation is possible in $O\left(\delta_{k}\right)$ time, if $f_{h_{1}, h_{2}, l}(t)$ and $b_{h_{1}, h_{2}, l}(t)$ are known.

Now we describe how we compute functions $f_{h_{1}, h_{2}, l}(t)$ and $b_{h_{1}, h_{2}, l}(t)$. Function $f_{h_{1}, h_{2}, l}(t)$ is computed by (8) from $f_{h_{1}-1}^{k}(t)$ if $h_{2}=h_{1}+l$, and from $f_{h_{1}, h_{2}-1, l}(t)$ if $h_{2}>h_{1}+l$. Function $b_{h_{1}, h_{2}, l}(t)$ is computed by (12) from $b_{h_{2}+1}^{k}(t)$ if $l=1$, and from $b_{h_{1}, h_{2}, l-1}(t)$ if $l>1$. These functions $f_{h_{1}-1}^{k}(t)$ and $b_{h_{2}+1}^{k}(t)$ are stored in memory as already mentioned. As we generate solutions in $N^{\text {intra }}$ in the order of Fig. 9, functions $f_{h_{1}, h_{2}-1, l}(t)$ and $b_{h_{1}, h_{2}, l-1}(t)$ are already available when they are needed for computing $f_{h_{1}, h_{2}, l}(t)$ and $b_{h_{1}, h_{2}, l}(t)$, respectively. From these arguments, we can conclude that $f_{h_{1}, h_{2}, l}(t)$ and $b_{h_{1}, h_{2}, l}(t)$ are computed in $O\left(\delta_{k}\right)$ time (i.e., the time to execute (8) and (12)).

Finally, we consider the space complexity of the above computation. We need $O\left(n_{k} \delta_{k}\right)$ space to store functions $f_{h}^{k}(t)$ and $b_{h}^{k}(t)$ for all customers in route $\sigma_{k}$. To compute $b_{h_{1}, h_{2}, l}(t)$, we only need to keep $b_{h_{1}, h_{2}, l-1}(t)$ in memory, which requires $O\left(\delta_{k}\right)$ space. To compute $f_{h_{1}, h_{2}, l}(t)$, we need to keep $f_{h_{1}, h_{2}-1, l}(t)$ for $l=1,2, \ldots, \min \left\{L_{\text {path }}^{\text {intra }}, n_{k}\right\}$, which requires $O\left(\min \left\{L_{\text {path }}^{\text {intra }}, n_{k}\right\} \cdot \delta_{k}\right)$ space. Therefore, the memory space required to store the necessary functions during the search of $N^{\text {intra }}(\boldsymbol{\sigma}, k)$ is $O\left(n_{k} \delta_{k}\right)$.

In conclusion, $\Delta p_{\text {sum }}$ of a solution in $N^{\text {cross }}, N^{2 \text { opt }^{*}}$ or $N^{\text {intra }}$ can be evaluated in $O\left(\sum_{k \in M^{\prime}} \delta_{k}\right)$ $=O(\delta)$ time provided that functions $f_{h}^{k}(t)$ and $b_{h}^{k}(t)$ for $h=1,2, \ldots, n_{k}$ and $k \in M$ are available, where $M^{\prime} \subseteq M\left(\left|M^{\prime}\right| \leq 2\right)$ is the set of indices of the relevant routes. The space complexity required for this computation is $O\left(\sum_{k \in M} n_{k} \delta_{k}\right)=O(n \delta)$. At the beginning of LS, we need to compute functions $f_{h}^{k}(t)$ and $b_{h}^{k}(t)$ for all $h=1,2, \ldots, n_{k}$ and $k \in M$, which is possible in $O\left(\sum_{k \in M} n_{k} \delta_{k}\right)=O(n \delta)$ time. We also need to recompute functions $f_{h}^{k}(t)$ and $b_{h}^{k}(t)$ for all $h=1,2, \ldots, n_{k}$ and $k \in M^{\prime}$, whenever the current solution is updated (i.e., the current round is 
over). This computation takes $O\left(\sum_{k \in M^{\prime}} n_{k} \delta_{k}\right)=O(n \delta)$ time. As $\left|N^{\text {cross }}\right|,\left|N^{2 \text { opt }^{*}}\right|$ and $\left|N^{\text {intra }}\right|$ are larger than $n$, the one-round times for $N^{\text {cross }}, N^{20 p t^{*}}$ and $N^{\text {intra }}$ with respect to $p_{\text {sum }}$ are $O\left(\left|N^{\text {cross }}\right| \delta\right), O\left(\left|N^{2 \mathrm{opt}^{*}}\right| \delta\right)$ and $O\left(\left|N^{\mathrm{intra}}\right| \delta\right)$, respectively, and the space complexity is $O(n \delta)$ for such neighborhoods.

We can further reduce the number of pieces in forward and backward minimum penalty functions. Since $f_{h}^{k}(t)$ (resp., $b_{h}^{k}(t)$ ) is nonincreasing (resp., nondecreasing), there are usually many pieces with considerably large value in $f_{h}^{k}(t)$ (resp., in $b_{h}^{k}(t)$ ) for small (resp., large) $t$. Such pieces will not be used in evaluating improved solutions. Therefore, we set a value INF and delete those pieces whose values over their intervals are not less than INF. We then add one piece whose interval is the union of the deleted intervals and function value is always INF. Even after this modification, we can compute the exact optimal time penalty $p_{\mathrm{sum}}^{*}\left(\sigma_{k}\right)$ if $p_{\mathrm{sum}}^{*}\left(\sigma_{k}\right)<\mathrm{INF}$ holds; otherwise we can conclude that $p_{\text {sum }}^{*}\left(\sigma_{k}\right) \geq$ INF holds. In our algorithm, INF is set to a sufficiently large value at the beginning of the local search. Then, whenever the current solution is improved during search, we set INF $:=\operatorname{cost}(\boldsymbol{\sigma})$ for the current solution $\boldsymbol{\sigma}$, which ensures that the algorithm never misses improved solutions. Though the worst case time complexity is not improved by this modification, actual computation usually becomes much faster.

\subsubsection{Evaluation of $\Delta q_{\text {sum }}$}

Let us consider the total amount of goods $\sum_{h=1}^{n_{k}} q_{\sigma_{k}(h)}$ to be delivered in a route $\sigma_{k}$. We define

$$
\begin{aligned}
& \gamma_{0}^{k}=0 \\
& \gamma_{h}^{k}=\sum_{l=1}^{h} q_{\sigma_{k}(l)}=\gamma_{h-1}^{k}+q_{\sigma_{k}(h)}, \quad h=1,2, \ldots, n_{k}
\end{aligned}
$$

for all $k \in M$, and store these values in memory during the search. Then, the sum $\sum_{l=h}^{h^{\prime}} q_{\sigma_{k}(l)}$ of the amount of goods for the $h$ th through the $h^{\prime}$ th customers can be computed in $O(1)$ time by $\gamma_{h^{\prime}}^{k}-\gamma_{h-1}^{k}$. Hence $\Delta q_{\text {sum }}$ is computed in $O(1)$ time for a solution in $N^{\text {cross }}$ or $N^{2 \mathrm{opt}^{*}}\left(\Delta q_{\text {sum }}\right.$ is always 0 for solutions in $N^{\text {intra }}$ ).

At the beginning of LS, we need to initialize all $\gamma_{h}^{k}$, which is possible in $O\left(\sum_{k \in M} n_{k}\right)=O(n)$ time. Whenever the current solution is updated, we also need to recompute $\gamma_{h}^{k}$ for all $h=$ $0,1, \ldots, n_{k}$ and $k \in M^{\prime}$, where $M^{\prime} \subseteq M\left(\left|M^{\prime}\right| \leq 2\right)$ is the set of indices of relevant routes. The time required for this is $O\left(\sum_{k \in M^{\prime}} n_{k}\right)=O(n)$. As sizes $\left|N^{\text {cross }}\right|,\left|N^{20 p t^{*}}\right|$ and $\left|N^{\text {intra }}\right|$ are larger than $n$, one-round times for $N^{\text {cross }}, N^{2 \text { opt* }}$ and $N^{\text {intra }}$ with respect to $q_{\text {sum }}$ are $O\left(\left|N^{\text {cross }}\right|\right)$, $O\left(\left|N^{2 \mathrm{opt}^{*}}\right|\right)$ and $O\left(\left|N^{\mathrm{intra}}\right|\right)$, respectively. The space complexity of the above computation is $O(n)$. 


\subsection{Search in the cyclic exchange neighborhood}

In this section, we introduce the improvement graph (Ahuja et al. 2002, Ahuja, Orlin and Sharma 2000 ), which is used to find an improved solution in $N^{\text {cyclic }}$. Then we propose a heuristic on how to search the improvement graph.

\subsubsection{Improvement graph}

For simplicity, we first describe the improvement graph in which acyclic exchange operations are not considered, and then later describe how acyclic exchange operations are treated. The improvement graph $G(\boldsymbol{\sigma})=(V(\boldsymbol{\sigma}), E(\boldsymbol{\sigma}))$ is defined for a feasible solution $\boldsymbol{\sigma}$. The set $V(\boldsymbol{\sigma})$ consists of nodes $v_{i l}\left(i=1,2, \ldots, n, l=1,2, \ldots, L^{\text {cyclic }}\right)$, where a node $v_{i l} \in V(\boldsymbol{\sigma})$ represents path $\psi_{i l}$ (i.e., the path of length $l$ from $i$ in $\left.\boldsymbol{\sigma}\right)$. A directed $\operatorname{arc} e_{i l i^{\prime} l^{\prime}}^{\mathrm{n}}\left(\right.$ resp., $\left.e_{i l i^{\prime} l^{\prime}}^{\mathrm{r}}\right) \in E(\boldsymbol{\sigma})$ from node $v_{i l}$ to $v_{i^{\prime} l^{\prime}}$ indicates that paths $\psi_{i l}$ and $\psi_{i^{\prime} l^{\prime}}$ are removed from routes $\sigma_{k[i]}$ and $\sigma_{k\left[i^{\prime}\right]}$, respectively, and $\psi_{i l}$ is inserted in place of $\psi_{i^{\prime} l^{\prime}}$ in $\sigma_{k\left[i^{\prime}\right]}$ with its visiting order unchanged (resp., reversed). Here $k[i]$ denotes the vehicle that visits customer $i$. That is, $e_{i l i^{\prime} l^{\prime}}^{\mathrm{n}}$ and $e_{i l i^{\prime} l^{\prime}}^{\mathrm{r}}$ represent normal and reverse insertions, respectively. Two $\operatorname{arcs} e_{i l i^{\prime} l^{\prime}}^{\mathrm{n}}$ and $e_{i l i^{\prime} l^{\prime}}^{\mathrm{r}}$ are in $E(\boldsymbol{\sigma})$ if and only if $k[i] \neq k\left[i^{\prime}\right]$ holds. Let $h_{i}$ be the index satisfying $\sigma_{k[i]}\left(h_{i}\right)=i$ for customer $i$, and define the following routes:

$$
\begin{aligned}
& \sigma_{i l i^{\prime} l^{\prime}}^{\mathrm{n}}=\left\langle\text { the depot } \rightarrow \sigma_{k\left[i^{\prime}\right]}\left(h_{i^{\prime}}-1\right), \sigma_{k[i]}\left(h_{i}\right) \rightarrow \sigma_{k[i]}\left(h_{i}+l-1\right), \sigma_{k\left[i^{\prime}\right]}\left(h_{i^{\prime}}+l^{\prime}\right) \rightarrow \text { the depot }\right\rangle, \\
& \sigma_{i l i^{\prime} l^{\prime}}^{\mathrm{r}}=\left\langle\text { the depot } \rightarrow \sigma_{k\left[i^{\prime}\right]}\left(h_{i^{\prime}}-1\right), \sigma_{k[i]}\left(h_{i}+l-1\right) \rightarrow \sigma_{k[i]}\left(h_{i}\right), \sigma_{k\left[i^{\prime}\right]}\left(h_{i^{\prime}}+l^{\prime}\right) \rightarrow \text { the depot }\right\rangle .
\end{aligned}
$$

Then, the costs of $\operatorname{arcs} e_{i l i^{\prime} l^{\prime}}^{\mathrm{n}}$ and $e_{i l i^{\prime} l^{\prime}}^{\mathrm{r}}$ are defined as $\operatorname{cost}\left(\sigma_{i l l^{\prime} l^{\prime}}^{\mathrm{n}}\right)-\operatorname{cost}\left(\sigma_{k\left[i^{\prime}\right]}\right)$ and $\operatorname{cost}\left(\sigma_{i l i^{\prime} l^{\prime}}^{\mathrm{r}}\right)-$ $\operatorname{cost}\left(\sigma_{k\left[i^{\prime}\right]}\right)$ (i.e., the cost increases on route $\sigma_{k\left[i^{\prime}\right]}$ by the insertions), respectively.

We call a directed cycle $C$ in the improvement graph $G(\boldsymbol{\sigma})$ subset-disjoint if $k[i] \neq k\left[i^{\prime}\right]$ holds for any pair of nodes $v_{i l}$ and $v_{i^{\prime} l^{\prime}}$ in $C$ (i.e., all the participating paths belong to different routes). We then call a subset-disjoint cycle a valid cycle if the sum of their arc costs is negative. As easily understood, there is one-to-one correspondence between solutions of cyclic exchange operations in $N^{\text {cyclic }}(\boldsymbol{\sigma})$ and subset-disjoint cycles in $G(\boldsymbol{\sigma})$, and the cost of a cycle represents the cost increase of the corresponding solution from that of the current solution $\boldsymbol{\sigma}$. Hence an improved solution in $N^{\text {cyclic }}(\boldsymbol{\sigma})$ can be found by a valid cycle in $G(\boldsymbol{\sigma})$.

We need only one of $e_{i l i^{\prime} l^{\prime}}^{\mathrm{n}}$ and $e_{i l i^{\prime} l^{\prime}}^{\mathrm{r}}$ with lower cost to find a valid cycle. Therefore, instead of having two $\operatorname{arcs} e_{i l i^{\prime} l^{\prime}}^{\mathrm{n}}$ and $e_{i l i^{\prime} l^{\prime}}^{\mathrm{r}}$, only one $\operatorname{arc} e_{i l i^{\prime} l^{\prime}}=\left(v_{i l}, v_{i^{\prime} l^{\prime}}\right)$, whose cost is

$$
c_{i l i^{\prime} l^{\prime}}=\min \left\{\operatorname{cost}\left(\sigma_{i l i^{\prime} l^{\prime}}^{\mathrm{n}}\right)-\operatorname{cost}\left(\sigma_{k\left[i^{\prime}\right]}\right), \operatorname{cost}\left(\sigma_{i l i^{\prime} l^{\prime}}^{\mathrm{r}}\right)-\operatorname{cost}\left(\sigma_{k\left[i^{\prime}\right]}\right)\right\}
$$

is included in $E(\boldsymbol{\sigma})$ for each ordered pair of $v_{i l}$ and $v_{i^{\prime} l^{\prime}}$.

We now describe how to deal with acyclic exchange operations in the improvement graph. We create nodes $v_{i 0}$ for $i=1,2, \ldots, n$ and $v_{00}^{k}$ for $k=1,2, \ldots, m$, where $v_{i 0}$ and $v_{00}^{k}$ correspond 
to empty paths $\psi_{i 0}$ and $\psi_{00}^{k}$, respectively. We also create a node $\hat{v}$. These nodes are connected by $\operatorname{arcs}\left(v_{i 0}, \hat{v}\right),\left(v_{00}^{k}, \hat{v}\right),\left(\hat{v}, v_{i^{\prime} l^{\prime}}\right),\left(v_{i^{\prime} l^{\prime}}, v_{i 0}\right)$ and $\left(v_{i^{\prime} l^{\prime}}, v_{00}^{k}\right)$ for $i, i^{\prime}=1,2, \ldots, n, l^{\prime}=1,2, \ldots, L^{\text {cyclic }}$ and $k=1,2, \ldots, m$. Let us define three routes $\sigma_{i^{\prime} l^{\prime}}, \sigma_{i^{\prime} l^{\prime} i 0}^{\mathrm{n}}$ and $\sigma_{i^{\prime} l^{\prime} i 0}^{\mathrm{r}}$ by

$$
\begin{aligned}
\sigma_{i^{\prime} l^{\prime}} & =\left\langle\text { the depot } \rightarrow \sigma_{k\left[i^{\prime}\right]}\left(h_{i^{\prime}}-1\right), \sigma_{k\left[i^{\prime}\right]}\left(h_{i^{\prime}}+l^{\prime}\right) \rightarrow \text { the depot }\right\rangle, \\
\sigma_{i^{\prime} l^{\prime} i 0}^{\mathrm{n}} & =\left\langle\text { the depot } \rightarrow \sigma_{k[i]}\left(h_{i}-1\right), \sigma_{k\left[i^{\prime}\right]}\left(h_{i^{\prime}}\right) \rightarrow \sigma_{k\left[i^{\prime}\right]}\left(h_{i^{\prime}}+l^{\prime}-1\right), \sigma_{k[i]}\left(h_{i}\right) \rightarrow \text { the depot }\right\rangle, \\
\sigma_{i^{\prime} l^{\prime} i 0}^{\mathrm{r}} & =\left\langle\text { the depot } \rightarrow \sigma_{k[i]}\left(h_{i}-1\right), \sigma_{k\left[i^{\prime}\right]}\left(h_{i^{\prime}}+l^{\prime}-1\right) \rightarrow \sigma_{k\left[i^{\prime}\right]}\left(h_{i^{\prime}}\right), \sigma_{k[i]}\left(h_{i}\right) \rightarrow \text { the depot }\right\rangle .
\end{aligned}
$$

Then the cost of $\operatorname{arc}\left(v_{i 0}, \hat{v}\right)$ is defined as 0 , that of $\operatorname{arc}\left(\hat{v}, v_{i^{\prime} l^{\prime}}\right)$ is defined as $\operatorname{cost}\left(\sigma_{i^{\prime} l^{\prime}}\right)-\operatorname{cost}\left(\sigma_{k\left[i^{\prime}\right]}\right)$, and that of $\operatorname{arc}\left(v_{i^{\prime} l^{\prime}}, v_{i 0}\right)$ is defined as

$$
\min \left\{\operatorname{cost}\left(\sigma_{i^{\prime} l^{\prime} i 0}^{\mathrm{n}}\right)-\operatorname{cost}\left(\sigma_{k[i]}\right), \operatorname{cost}\left(\sigma_{i^{\prime} l^{\prime} i 0}^{\mathrm{r}}\right)-\operatorname{cost}\left(\sigma_{k[i]}\right)\right\} .
$$

The costs of the $\operatorname{arcs}\left(v_{00}^{k}, \hat{v}\right)$ and $\left(v_{i^{\prime} l^{\prime}}, v_{00}^{k}\right)$ are similarly defined. Now a cycle $C$ in the modified improvement graph represents acyclic exchange operations if and only if $C$ contains $\hat{v}$. Thus the graph contains a valid cycle if and only if an improved solution (either cyclic or acyclic exchange operations) exists in $N^{\text {cyclic }}(\boldsymbol{\sigma})$.

Let us now consider the size of the improvement graph and time complexity to construct it. Since there are $O\left(L^{\text {cyclic }} n\right)$ paths to be considered for the cyclic exchange operation, $|V(\boldsymbol{\sigma})|=$ $O\left(L^{\text {cyclic }} n\right)$ and $|E(\boldsymbol{\sigma})|=O\left(\left(L^{\text {cyclic }} n\right)^{2}\right)$ hold. From the discussion in Section 4.1, it is not difficult to see that the cost of an arc in $E(\boldsymbol{\sigma})$ can be computed in $O\left(\delta_{k[i]}+\delta_{k\left[i^{\prime}\right]}\right)=O(\delta)$ time, provided that $f_{h}^{k}(t), b_{h}^{k}(t)$ and $\gamma_{h}^{k}$ are known for $h=1,2, \ldots, n_{k}$ and $k \in M$, and the order of computing the arc costs is carefully designed. As all $f_{h}^{k}(t)$ and $b_{h}^{k}(t)$ (resp., $\gamma_{h}^{k}$ ) are computed in $O(n \delta)$ (resp., $O(n))$ time, the construction of $G(\boldsymbol{\sigma})$ is possible in $O\left(\left(L^{\text {cyclic }} n\right)^{2} \delta\right)$ time. The space complexity to compute arc costs is $O(n \delta)$ (needed to store functions $f_{h}^{k}(t)$ and $b_{h}^{k}(t)$ ), and the space to store graph $G(\boldsymbol{\sigma})$ is $O\left(\left(L^{\text {cyclic }} n\right)^{2}\right)$.

\subsubsection{A heuristic algorithm to find a valid cycle}

As the problem of finding a valid cycle in a general improvement graph of this type is known to be NP-hard (Thompson and Orlin 1989), we develop a heuristic algorithm, which is not guaranteed to find a valid cycle even if it exists. Our algorithm is based on the labeling algorithm for the shortest path problem.

We first briefly summarize the labeling algorithm in $G(\boldsymbol{\sigma})$. Let $c_{u v}$ denote the cost of an $\operatorname{arc}(u, v) \in E(\boldsymbol{\sigma})$. The cost of a path in $G(\boldsymbol{\sigma})$ is the sum of $c_{u v}$ of $\operatorname{arcs}(u, v)$ in the path, and the length of a path is the number of vertices in the path. The shortest path problem asks to find a path of minimum cost from a specified node $v_{s} \in V(\boldsymbol{\sigma})$ to every other node in $V(\boldsymbol{\sigma})$. The algorithm computes labels $\operatorname{label}(v, l)$ for all $v \in V(\boldsymbol{\sigma})$, from $l=1$ to $|V(\boldsymbol{\sigma})|$, where label $(v, l)$ stores the minimum cost among all directed paths from $v_{s}$ to $v$ of length at most $l$. If 
$\operatorname{label}(v, l)=\infty$, no directed path of length at most $l$ from $v_{s}$ to $v$ is found yet. We call the phase of computing $l a b e l(v, l)$ for all $v \in V(\boldsymbol{\sigma})$ the $l$ th phase. The algorithm also maintains $\operatorname{prev}(v)$ for all $v \in V(\boldsymbol{\sigma})$, which represents the previous node of $v$ in the directed path to $v$ of cost label $(v, l)$. The algorithm starts by setting $\operatorname{label}\left(v_{s}, 1\right):=0$ and $\operatorname{label}(v, 1):=\infty$ for all $v \in V(\boldsymbol{\sigma}) \backslash\left\{v_{s}\right\}$. At the beginning of the $l$ th phase with $l \geq 2$, labels are set $\operatorname{label}(v, l):=\operatorname{label}(v, l-1)$ for all $v \in V(\boldsymbol{\sigma})$. Then, for every arc $(u, v)$, if $\operatorname{label}(u, l-1)+c_{u v}<\operatorname{label}(v, l)$ holds, $\operatorname{label}(v, l)$ is updated to $\operatorname{label}(u, l-1)+c_{u v}$ and $\operatorname{prev}(v)$ is updated to $u$. This step is called the label update step. The $l$ th phase ends if all arcs are checked. The algorithm stops when $\operatorname{label}(v, l-1)=\operatorname{label}(v, l)$ holds for all $v \in V(\boldsymbol{\sigma})$ at the end of the $l$ th phase, or it reaches the end of the $|V(\boldsymbol{\sigma})|$ th phase.

The algorithm is usually implemented on a node set $\hat{V}$, called the active nodes, which is set to $\left\{v_{s}\right\}$ in the second phase, and is the set of those $v$ satisfying label $(v, l-1)<\operatorname{label}(v, l-2)$ in the $l$ th phase with $l \geq 3$. Let $E_{v}$ be the set of arcs whose tail vertex is $v$. In the $l$ th phase $(l \geq 2)$, only the $\operatorname{arcs}$ in $\hat{E}=\bigcup_{v \in \hat{V}} E_{v}$ are scanned for the label update step, since $\operatorname{label}(u, l-1)+c_{u v} \geq \operatorname{label}(v, l)$ holds for all $\operatorname{arcs}(u, v)$ not in $\hat{E}$.

We make some modifications on the above labeling algorithm. The set of active nodes is further reduced as follows. We only consider those $\operatorname{arcs}(u, v)$ with $\operatorname{label}(u, l-1)<0$ for the label update steps in the $l$ th phase. That is, we delete those nodes $u$ with $\operatorname{label}(u, l-1) \geq 0$ from $\hat{V}$. Then the set of the reduced active nodes is denoted $\tilde{V}$.

We repeat the algorithm by regarding every node as the source. Recall that we only consider valid cycles of length at most $\nu^{\text {cyclic }}$ (Section 3.1.2). This parameter is motivated by the fact that longer cycles are less likely to be subset-disjoint. As a cycle of length more than $m$ will not be subset-disjoint, we assume that $\nu^{\text {cyclic }} \leq m$ holds. Our algorithm finishes the search with the current source node and exits to a further search with another source node, if either of the following two conditions holds: (1) the algorithm reaches the $\left(\nu^{\text {cyclic }}+1\right)$ st phase, $(2)$ the set of active nodes $\tilde{V}$ becomes empty. Finally, the entire algorithm halts either (1) when a valid cycle is identified, or (2) when the search is completed with all source nodes in $V(\boldsymbol{\sigma})$.

Further modification is that the label update step is executed only if the resulting path is subset-disjoint. In order to check whether a path considered in each label update step is subsetdisjoint or not in $O(1)$ time, we maintain $\operatorname{set}(v, k)$ for all $v \in V(\boldsymbol{\sigma})$ and $k \in M$. Let $P[v]$ denote the path from $v_{s}$ to $v$ obtained by tracing $\operatorname{prev}(v)$ from node $v$ back to $v_{s}$, and let $k_{v}$ be the index of the route that contains the path (of customers) corresponding to node $v \in V(\boldsymbol{\sigma})$. The value of $\operatorname{set}(v, k)$ is 1 , if $P[v]$ contains a vertex $u$ satisfying $k_{u}=k$, and 0 otherwise. Then the label update step for an $\operatorname{arc}(u, v) \in \tilde{E}$ is executed only if $\operatorname{set}\left(u, k_{v}\right)=0$ holds (i.e., the new path $P[v]$ is assured to be subset-disjoint after the label update step). At the beginning of the $l$ th phase with $l \geq 3, \operatorname{set}(v, k)$ are updated for those $v \in \tilde{V}$ by first resetting $\operatorname{set}(v, k):=0$ for all $k$ and then setting $\operatorname{set}\left(v, k_{u}\right):=1$ for all $u$ in $P[v]$. 
The final modification is that the algorithm checks if $\operatorname{label}(v, l)+c_{v v_{s}}<0$ holds for some $v \in \tilde{V}$, at the end of the $l$ th phase. If such a $v$ is found, the resulting cycle $\left(P[v], v_{s}\right)$ is a valid cycle.

The modification of active nodes from $\hat{V}$ to $\tilde{V}$ is motivated by the following simple well-known lemma (see, e.g., Lin and Kernighan 1973).

Lemma 4.1 If $\sum_{i=1}^{l} c_{i}<0$ holds, then there exists a $j$ such that $\sum_{i=j}^{k} c_{i}<0$ holds for all $k=j, j+1, \ldots, l$, and $\sum_{i=1}^{k} c_{i}+\sum_{i=j}^{l} c_{i}<0$ holds for all $k=1,2, \ldots, j-1$.

Proof: Let $k^{*}$ be the largest index among those $k$ that maximizes $\sum_{i=1}^{k} c_{i}$. Then $\sum_{i=1}^{k} c_{i}+$ $\sum_{i=k^{*}+1}^{l} c_{i} \leq \sum_{i=1}^{k^{*}} c_{i}+\sum_{i=k^{*}+1}^{l} c_{i}<0$ holds if $1 \leq k \leq k^{*}$, and $\sum_{i=k^{*}+1}^{k} c_{i}=\sum_{i=1}^{k} c_{i}-\sum_{i=1}^{k^{*}} c_{i}<$ 0 holds if $k^{*}+1 \leq k \leq l$. Hence $j=k^{*}+1$ satisfies the conditions in the lemma.

This lemma indicates that, in a valid cycle $C$, there exists a vertex $v \in C$ such that the costs of all the directed paths in $C$ starting from $v$ are negative. Thus, although only maintaining labels with negative cost might miss some valid cycles in the search with the current source node, the algorithm will eventually find such valid cycles starting from other source node.

The algorithm, called FVC, to find a valid cycle in a given directed graph $G(\boldsymbol{\sigma})$ is summarized as follows. In the algorithm, $\hat{V}$ is the sets of vertices whose labels have been improved in the label update step of the current phase, $\tilde{V}$ is the subset of $\hat{V}$ whose labels are negative, and $\tilde{E}$ is $\bigcup_{v \in \tilde{V}} E_{v}$

Algorithm $\mathbf{F V C}(G(\boldsymbol{\sigma})=(V(\boldsymbol{\sigma}), E(\boldsymbol{\sigma})))$

Step 1 Let $U:=V(\boldsymbol{\sigma})$.

Step 2 Choose a node $v_{s} \in U$ and let $U:=U \backslash\left\{v_{s}\right\}$, label $(u, 1):=\infty$ for all $u \in V(\boldsymbol{\sigma}) \backslash\left\{v_{s}\right\}$, $\operatorname{label}\left(v_{s}, 1\right):=0, \tilde{V}:=\left\{v_{s}\right\}, \hat{V}:=\emptyset, \tilde{E}:=E_{v_{s}}, \operatorname{set}(v, k):=0$ for all $v \in V(\boldsymbol{\sigma}) \backslash\left\{v_{s}\right\}$ and $k \in M, \operatorname{set}\left(v_{s}, k_{v_{s}}\right):=1$ and $l:=2$.

Step 3 If $l>\nu^{\text {cyclic }}$, go to Step 8. Otherwise, let $\operatorname{label}(v, l):=\operatorname{label}(v, l-1)$ for all $v \in V(\boldsymbol{\sigma})$.

Step 4 Choose an arc $e_{u v}=(u, v) \in \tilde{E}$ and let $\tilde{E}:=\tilde{E} \backslash\left\{e_{u v}\right\}$. If $\operatorname{both} \operatorname{set}\left(u, k_{v}\right)=0$ and $\operatorname{label}(u, l-1)+c_{u v}<\operatorname{label}(v, l)$ hold, let $\operatorname{label}(v, l):=\operatorname{label}(u, l-1)+c_{u v}, \operatorname{prev}(v):=u$ and $\hat{V}:=\hat{V} \cup\{v\}$.

Step 5 If $\tilde{E} \neq \emptyset$, return to Step 4 .

Step 6 Let $\operatorname{set}(v, k):=0$ for all $v \in \tilde{V}$ and $k \in M$. Then let $\tilde{V}:=\emptyset$.

Step 7 Choose a node $v \in \hat{V}$ and let $\hat{V}:=\hat{V} \backslash\{v\}$. If label $(v, l) \geq 0$ holds, proceed to Step 8 . If label $(v, l)+c_{v v_{s}}<0$ holds, output the corresponding valid cycle and halt. Otherwise, let $\tilde{V}:=\tilde{V} \cup\{v\}, \tilde{E}:=\tilde{E} \cup E_{v}$ and $\operatorname{set}\left(v, k_{u}\right):=1$ for all $u$ in $P[v]$.

Step 8 If $\hat{V} \neq \emptyset$, return to Step 7. Otherwise, if $\tilde{V} \neq \emptyset$, let $l:=l+1$ and return to Step 3 .

Step 9 If $U=\emptyset$, halt. Otherwise, return to Step 2. 
Let us consider the time complexity of algorithm FVC. The number of repetitions of the loop from Step 2 through Step 9 is $O(|V(\boldsymbol{\sigma})|)$. The initialization in Step 2 requires $O(|V(\boldsymbol{\sigma})| m)$ time for letting $\operatorname{set}(v, k):=0$ for all $u \in V(\boldsymbol{\sigma})$ and $k \in M$. The loop of Steps 3 to 8 is repeated $O\left(\nu^{\text {cyclic }}\right)$ times. In Step 3, it takes $O(|V(\boldsymbol{\sigma})|)$ time to initialize labels $\operatorname{label}(v, l)$ for all $v \in V(\boldsymbol{\sigma})$. Steps 4 and 5 are repeated $O(|\tilde{E}|)$ times, and take $O(1)$ time for each execution. Then, Step 6 requires $O(|\tilde{V}| l)$ time, since resetting $\operatorname{set}(v, k):=0$ for all $k \in M$ can be done by tracing the vertices in the path $P[v]$ for all $v \in \tilde{V}$. For the same reason, Step 7 is carried out in $O(l)$ time to update $\operatorname{set}(v, k)$. Step 8 is executed in $O(1)$ time. The loop of Steps 7 and 8 is repeated $O(|\hat{V}|)$ times. Consequently the total time required for FVC is $O\left(|V(\boldsymbol{\sigma})|^{2} m+\nu^{\text {cyclic }}|V(\boldsymbol{\sigma})||E(\boldsymbol{\sigma})|+\left(\nu^{\text {cyclic }}\right)^{2}|V(\boldsymbol{\sigma})|^{2}\right)=O\left(\nu^{\text {cyclic }}\left(L^{\text {cyclic }} n\right)^{3}\right)$, since $m \leq n$, $|E(\boldsymbol{\sigma})|=O\left(\left(L^{\text {cyclic }} n\right)^{2}\right)$ and $|V(\boldsymbol{\sigma})|=O\left(L^{\text {cyclic }} n\right)$ hold. This complexity seems rather large; however, its actual computational time is usually much smaller, since the sizes of sets $\tilde{E}, \tilde{V}$ and $\hat{V}$ tend to decrease rapidly as $l$ increases.

\subsection{Time-oriented neighbor-lists}

In this section, we propose the time-oriented neighbor-lists to prune the neighborhood search heuristically. A similar technique, called the neighbor-lists, was successfully applied to TSP (Johnson and McGeoch 1997) and VRP (e.g., Park, Okano and Imai 2000), in which the neighbor-list of customer $i$ is determined on the basis of the distance from $i$. However, this is not appropriate for VRPGTW, since we should also take into account the start time of service at $i$.

Recall that all vehicles can leave the depot after time 0. Here we also assume that it is desirable to return to the depot before time $w_{0}^{\mathrm{d}}$. We partition the period of length $w_{0}^{\mathrm{d}}$ into $\nu^{\text {nlist }}$ (a parameter) intervals, and let $\rho=w_{0}^{\mathrm{d}} / \nu^{\text {nlist }}$. For each customer $i$, we construct $\nu^{\text {nlist }}$ timeoriented neighbor-lists, nlist $_{i}^{\phi}=\left(\operatorname{nlist}_{i}^{\phi}[1], \operatorname{nlist}_{i}^{\phi}[2], \ldots\right.$, nlist $\left._{i}^{\phi}[n]\right)$, for $\phi=1,2, \ldots, \nu^{\text {nlist }}$, where customer nlist ${ }_{i}^{\phi}[l]$ with a smaller $l$ is more desirable to be served immediately after $i$, provided that $\phi$ is the largest index satisfying $(\phi-1) \rho \leq s_{i}$. The desirability of a customer $j$ to be scheduled immediately after $i$ is determined in the increasing order of

$$
\operatorname{key}_{i}^{\phi}[j]= \begin{cases}d_{i j}+\min _{0 \leq t \leq \mu^{\text {nlist }} W} p_{j}((\phi-1) \rho+t), & \text { if } u_{i}+t_{i j} \leq \mu^{\text {nlist }} W \\ +\infty, & \text { otherwise }\end{cases}
$$

where $W=m w_{0}^{\mathrm{d}} / n$ and $\mu^{\text {nlist }}$ is a parameter. In other words, nlist ${ }_{i}^{\phi}[l]$ is the customer $j$ with the $l$ th smallest value of $\operatorname{key}_{i}^{\phi}[j]$. The structure of the time-oriented neighbor-lists is illustrated in Fig. 10.

(Figure 10) 
The meaning of (14) is explained as follows. If time $u_{i}+t_{i j}$ is large, serving $j$ immediately after $i$ is not desirable, since it will delay the service of subsequent customers. Therefore we set $\operatorname{key}_{i}^{\phi}[j]$ to $+\infty$ if $u_{i}+t_{i j}>\mu^{\text {nlist }} W$, where $W$ is a rough estimate of the average time spent for one customer (i.e., $s_{\sigma_{k}(h+1)}-s_{\sigma_{k}(h)}$ ), and $\mu^{\text {nlist }}$ is set to 3 in our experiment. For those $j$ with $u_{i}+t_{i j} \leq \mu^{\text {nlist }} W$, the first term in (14) is the distance from customer $i$ to customer $j$, while the second term represents the minimum time penalty of customer $j$ if it is served in the time interval of $\left[(\phi-1) \rho,(\phi-1) \rho+\mu^{\text {nlist }} W\right]$.

Before proceeding further, we comment on parameter $\nu^{\text {nlist }}$. It might be natural to set $\nu^{\text {nlist }}$ proportional to $n / m$ based on the same consideration as $W$; however, large memory space is necessary if $\nu^{\text {nlist }}$ is set large. We therefore set $\nu^{\text {nlist }}$ small to keep the memory requirement reasonable.

Let us now consider the time complexity of constructing the time-oriented neighbor-lists. The computation of $\operatorname{key}_{i}^{\phi}[j]$ requires $O\left(\delta^{(j)}\right)$ time $\left(\delta^{(j)}\right.$ was defined in Section 2). Therefore the computation of $\operatorname{key}_{i}^{\phi}[j]$ for all $j=1,2, \ldots, n, j \neq i$ requires $\sum_{j=1}^{n} O\left(\delta^{(j)}\right)=O(\delta)$ time. It follows that the construction of nlist ${ }_{i}^{\phi}$ for each of $i=1,2, \ldots, n$ and $\phi=1,2, \ldots, \nu^{\text {nlist }}$ requires $O(\delta+n \log n)$ time; hence $O\left(n \nu^{\text {nlist }}(\delta+n \log n)\right)$ in total.

We now describe how we use the time-oriented neighbor-lists for the search in $N^{20 p t}{ }^{*}$ and $N^{\text {cross }}$. We first explain the case of $N^{20 p t^{*}}$. For a customer $i=\sigma_{k}\left(h_{1}^{k}-1\right)$ (see Fig. 5), the customers selected for $\sigma_{k^{\prime}}\left(h_{1}^{k^{\prime}}\right)$ are nlist ${ }_{i}^{\phi}[l], l=1,2, \ldots, L_{2 \mathrm{opt}^{*}}^{\text {nlist }}\left(L_{2 \mathrm{opt}^{*}}^{\text {nlist }}\right.$ is a parameter $)$, where $\phi$ is fixed to $\phi=\left\lfloor s_{i} / \rho\right\rfloor+1$. Similarly, in the case of $N^{\text {cross }}$, for a customer $i=\sigma_{k}\left(h_{1}^{k}-1\right.$ ) (see Fig. 4), customers selected for $\sigma_{k^{\prime}}\left(h_{1}^{k^{\prime}}\right)$ are $\operatorname{nlist}_{i}^{\phi}[l], l=1,2, \ldots, L_{\text {cross }}^{\text {nlist }}$ ( $L_{\text {cross }}^{\text {nlist }}$ is a parameter), where $\phi$ is fixed to $\phi=\left\lfloor s_{i} / \rho\right\rfloor+1$. Then, all possible values for $h_{2}^{k}$ and $h_{2}^{k^{\prime}}$ (i.e., $h_{2}^{k}=h_{1}^{k}, h_{1}^{k}+$ $\left.1, \ldots, h_{1}^{k}+L^{\text {cross }}, h_{2}^{k^{\prime}}=h_{1}^{k^{\prime}}, h_{1}^{k^{\prime}}+1, \ldots, h_{1}^{k^{\prime}}+L^{\text {cross }}\right)$ are considered for each pair of $h_{1}^{k}$ and $h_{1}^{k^{\prime}}$. We do not use the time-oriented neighbor-lists in the search of $N^{\text {intra }}$, since it seems impossible to keep the time complexity of computing the minimum time penalty to $O(\delta)$ if this modification is added.

By using the time-oriented neighbor-lists, the neighborhood size of $N^{\text {cross }}$ is reduced from $O\left(\left(L^{\text {cross }}\right)^{2} n^{2}\right)$ to $O\left(\left(L^{\text {cross }}\right)^{2} n L_{\text {cross }}^{\text {nlist }}\right)$, and that of $N^{2 \mathrm{opt}^{*}}$ is reduced from $O\left(n^{2}\right)$ to $O\left(n L_{2 \mathrm{opt}}^{\text {nlist }}\right)$. We set $L_{2 \mathrm{opt}^{*}}^{\text {nlist }}:=\min \left\{n,\left(L^{\text {cross }}\right)^{2} L_{\text {cross }}^{\text {nlist }}\right\}$ in our algorithm so that the size of $N^{20 p t^{*}}$ becomes $O\left(\min \left\{n^{2},\left(L^{\text {cross }}\right)^{2} n L_{\text {cross }}^{\text {nlist }}\right\}\right)$. This parameter setting does not affect the overall neighborhood size, since the size of $N^{\text {cross }}$ is already $O\left(\left(L^{\text {cross }}\right)^{2} n L_{\text {cross }}^{\text {nlist }}\right)$.

\subsection{Other speedup techniques}

We explain two other techniques to speed up the local search. First, we maintain tables $I^{\text {cross }}\left(k, k^{\prime}\right), I^{2 \operatorname{opt}^{*}}\left(k, k^{\prime}\right)$ and $I^{\text {intra }}(k)$ for $k, k^{\prime} \in M$ so that we do not search the regions where no improvement is expected. We set $I^{\text {cross }}\left(k, k^{\prime}\right):=0$ whenever the algorithm finds no improvement 
in $N^{\text {cross }}\left(\boldsymbol{\sigma}, k, k^{\prime}\right)$, and set $I^{\text {cross }}\left(k, k^{\prime}\right):=1$ whenever an improvement occurs by any neighborhood operation on either $\sigma_{k}$ or $\sigma_{k^{\prime}}$. Tables $I^{2 \operatorname{opt}^{*}}\left(k, k^{\prime}\right)$ and $I^{\text {intra }}(k)$ are defined similarly. Then, we search $N^{\text {cross }}\left(\boldsymbol{\sigma}, k, k^{\prime}\right)$ only if $I^{\text {cross }}\left(k, k^{\prime}\right)=1$ holds. Similarly, we search $N^{2 o p t *}\left(\boldsymbol{\sigma}, k, k^{\prime}\right)$ (resp., $N^{\text {intra }}(\boldsymbol{\sigma}, k)$ ) only if $I^{2 \text { opt* }^{*}}\left(k, k^{\prime}\right)=1$ (resp., $I^{\text {intra }}(k)=1$ ) holds.

The second idea is to improve the search in the cyclic exchange neighborhood. The construction of the improvement graph is the most expensive part. (Though the worst case time complexity of algorithm FVC of Section 4.2.2 seems to become more expensive if $\delta=o\left(\nu^{\text {cyclic }} L^{\text {cross }} n\right)$ hold, its actual computational time is usually much smaller than that of the construction of the improvement graph.) To alleviate this, we do not construct the whole improvement graph from scratch. This is motivated by the fact that there may be some routes not modified during the search after the previous construction. In such a case, the costs of arcs between such routes do not change. For this, we maintain a table $I^{\text {cyclic }}(k)$ for $k \in M$. The algorithm sets $I^{\text {cyclic }}(k):=0$ for all $k \in M$ whenever the improvement graph is constructed. Then, if route $k$ is involved in the update of solutions (by any neighborhood operation), the algorithm sets $I^{\text {cyclic }}(k):=1$. In the reconstruction, the cost of an $\operatorname{arc}(u, v)$ is updated if and only if $I^{\text {cyclic }}\left(k_{u}\right)=1$ or $I^{\text {cyclic }}\left(k_{v}\right)=1$ holds.

Finally, we find more than one valid cycle in the improvement graph by applying algorithm FVC repeatedly. This is motivated by the fact that the costs on many arcs remain unchanged even after a cyclic exchange operation is applied. It is executed as follows. If a solution is improved by a cyclic exchange operation, we remove from the improvement graph those arcs whose head or tail node is in those routes involved in the update of the solution. Then, algorithm FVC is again applied to the reduced improvement graph. This procedure is repeated until either (1) FVC is unable to find a valid cycle, or (2) the number of the routes not involved in these updates of solutions becomes less than two. Feasible cyclic exchange operations always correspond to the valid cycles found during the above repeated calls to FVC, since $k_{u} \neq k_{v}$ holds for any two vertices $u$ and $v$ in these valid cycles.

\section{$5 \quad$ Metaheuristic algorithms}

In this section, we describe three metaheuristic algorithms, the multi-start local search (MLS) (Lin 1965, Lin and Kernighan 1973, Reiter and Sherman 1965), the iterated local search (ILS) (Johnson 1990, Martin, Otto and Felten 1991, Martin, Otto and Felten 1992), and the adaptive multi-start local search (AMLS) (Boese, Kahng and Muddu 1994, Taillard et al. 1997). All of these algorithms are based on the LS described so far. We describe how to generate initial solutions within their frameworks. 


\subsection{The multi-start local search and iterated local search}

In the multi-start local search (MLS), LS is repeatedly applied from a number of initial solutions and the best solution found during the entire search is output. Algorithm MLS is summarized as follows.

\section{Algorithm MLS}

Step 1 Generate an initial solution $\sigma^{0}$ and let $\boldsymbol{\sigma}^{\text {best }}:=\boldsymbol{\sigma}^{0}$.

Step 2 Improve $\boldsymbol{\sigma}^{0}$ by LS; i.e., $\boldsymbol{\sigma}:=\mathrm{LS}\left(\boldsymbol{\sigma}^{0}\right)$.

Step 3 If a solution $\boldsymbol{\sigma}^{\prime}$ satisfying besteval $\left(\boldsymbol{\sigma}^{\prime}\right)<$ besteval $\left(\boldsymbol{\sigma}^{\text {best }}\right)$ is found during the LS in Step 2, let $\boldsymbol{\sigma}^{\text {best }}:=\boldsymbol{\sigma}^{\prime}$.

Step 4 If some stopping criterion is satisfied, output $\boldsymbol{\sigma}^{\text {best }}$ and halt; otherwise generate a solution $\sigma^{0}$ and return to Step 2.

In the computational experiment in Section 6, the initial solutions of MLS are generated randomly.

The iterated local search (ILS) is a variant of MLS, in which initial solutions are generated by slightly perturbing a solution $\boldsymbol{\sigma}^{\text {seed }}$, which is a good (not necessarily the best) solution found during the search. The algorithm of ILS is summarized as follows.

\section{Algorithm ILS}

Step 1 Generate an initial solution $\boldsymbol{\sigma}^{0}$, and let $\boldsymbol{\sigma}^{\text {seed }}:=\boldsymbol{\sigma}^{0}$ and $\boldsymbol{\sigma}^{\text {best }}:=\boldsymbol{\sigma}^{0}$.

Step 2 Improve $\boldsymbol{\sigma}^{0}$ by LS; i.e., let $\boldsymbol{\sigma}:=\mathrm{LS}\left(\boldsymbol{\sigma}^{0}\right)$.

Step 3 If a solution $\boldsymbol{\sigma}^{\prime}$ satisfying besteval $\left(\boldsymbol{\sigma}^{\prime}\right)<\operatorname{besteval}\left(\boldsymbol{\sigma}^{\text {best }}\right)$ is found during the LS in Step 2, let $\boldsymbol{\sigma}^{\text {best }}:=\boldsymbol{\sigma}^{\prime}$.

Step 4 If $\operatorname{cost}(\boldsymbol{\sigma}) \leq \operatorname{cost}\left(\boldsymbol{\sigma}^{\text {seed }}\right)$, let $\boldsymbol{\sigma}^{\text {seed }}:=\boldsymbol{\sigma}$.

Step 5 If some stopping criterion is satisfied, output $\boldsymbol{\sigma}^{\text {best }}$ and halt; otherwise generate a solution $\boldsymbol{\sigma}^{0}$ by slightly perturbing $\boldsymbol{\sigma}^{\text {seed }}$ and return to Step 2 .

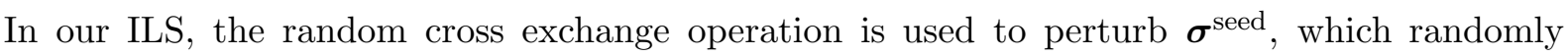
chooses two paths from two routes with no restriction on the length of the paths and exchanges them. The algorithm executes the random cross exchange operation $\nu_{\mathrm{ptb}}^{\mathrm{ILS}}$ (a parameter) times on $\boldsymbol{\sigma}^{\text {seed }}$ to generate a new initial solution $\boldsymbol{\sigma}^{0}$ in Step 5 .

\subsection{The adaptive multi-start local search}

AMLS maintains a set of solutions $P=\left\{\boldsymbol{\sigma}^{1}, \boldsymbol{\sigma}^{2}, \ldots, \boldsymbol{\sigma}^{|P|}\right\}$ during the search, where $|P| \leq \nu_{\text {pop }}^{\text {AMLS }}$ holds $\left(\nu_{\mathrm{pop}}^{\mathrm{AMLS}}\right.$ is a parameter). Solutions in $P$ are selected from the locally optimal solutions found in the previous search, and are used to generate the initial solution for the next local 
search. We first describe how we generate the initial solution for the next LS, then describe how we initialize $P$ and update it during the search, and finally summarize the outline of algorithm AMLS.

Let the current $P$ consist of $\boldsymbol{\sigma}^{\lambda}=\left(\sigma_{1}^{\lambda}, \sigma_{2}^{\lambda}, \ldots, \sigma_{m}^{\lambda}\right), \lambda=1,2, \ldots,|P|$, and $R[P]$ be the set of $|P| m$ routes $\left\{\sigma_{1}^{1}, \sigma_{2}^{1}, \ldots, \sigma_{m}^{1}, \sigma_{1}^{2}, \sigma_{2}^{2}, \ldots, \sigma_{m}^{2}, \ldots, \sigma_{1}^{|P|}, \sigma_{2}^{|P|}, \ldots, \sigma_{m}^{|P|}\right\}$. The initial solution $\boldsymbol{\sigma}^{0}=\left(\sigma_{1}^{0}, \sigma_{2}^{0}, \ldots, \sigma_{m}^{0}\right)$ is constructed by selecting routes $\sigma$ from $R[P]$ one by one. Let $\boldsymbol{\sigma}^{0}$ be the current solution being constructed, where some routes in $\sigma^{0}$ may be empty, and let $\sigma$ be a route in $R[P] . V_{\sigma}$ and $V_{\boldsymbol{\sigma}^{0}}$ denote the sets of customers in route $\sigma$ and solution $\boldsymbol{\sigma}^{0}$, respectively. Then let

$$
c_{\text {gen }}\left(\sigma, \boldsymbol{\sigma}^{0}\right)= \begin{cases}\frac{\operatorname{cost}(\sigma)}{\mid V_{\sigma} \backslash V_{\boldsymbol{\sigma}^{0}}}, & \text { if }\left|V_{\sigma} \backslash V_{\boldsymbol{\sigma}^{0}}\right| \neq 0 \\ \infty, & \text { otherwise. }\end{cases}
$$

Cost $c_{\text {gen }}\left(\sigma, \boldsymbol{\sigma}^{0}\right)$ measures the attractiveness of route $\sigma$ with respect to the current solution $\boldsymbol{\sigma}^{0}$ under consideration. If $\left|V_{\sigma} \backslash V_{\boldsymbol{\sigma}^{0}}\right|=0$ holds, all customers in $\sigma$ have already been assigned to $\boldsymbol{\sigma}^{0}$ and hence it is useless to insert $\sigma$ into $\boldsymbol{\sigma}^{0}$, and hence $c_{\text {gen }}$ is set $\infty$. Otherwise, $c_{\text {gen }}\left(\sigma, \boldsymbol{\sigma}^{0}\right)$ indicates the average cost to visit a customer not in $\sigma^{0}$.

All the routes in $\sigma^{0}$ are initially set to be empty, and then the route selection step is executed $m$ times. In the $k$ th route selection step, the route $\sigma^{*}$ with the minimum $c_{\text {gen }}\left(\sigma, \sigma^{0}\right)$ is selected from $R[P]$ and we set $\sigma_{k}^{0}:=\sigma^{*}$. Here we restrict $\sigma^{0}$ to contain at most $\left\lceil m / \nu_{\text {pop }}^{\text {AMLS }}\right\rceil$ routes from one $\boldsymbol{\sigma}^{\lambda}$ in $P$ to keep its diversity. This is achieved simply by setting $c_{\text {gen }}\left(\sigma, \boldsymbol{\sigma}^{0}\right):=\infty$ for all $\sigma \in \boldsymbol{\sigma}^{\lambda}$ when $\boldsymbol{\sigma}^{0}$ contains $\left\lceil m / \nu_{\text {pop }}^{\mathrm{AMLS}}\right\rceil$ routes from $\boldsymbol{\sigma}^{\lambda}$. After $m$ route selection steps, some customers may be assigned to more than one route in $\boldsymbol{\sigma}^{0}$. We call them duplicate customers, and apply the following customer deletion steps. Let $i$ be a duplicate customer, which is assigned to $Z$ routes $\sigma_{k_{1}}^{0}, \sigma_{k_{2}}^{0}, \ldots, \sigma_{k_{Z}}^{0}$. We define $\hat{\sigma}_{k_{z}}$ to be the route obtained by deleting customer $i$ from $\sigma_{k_{z}}^{0}$. Then we set $\sigma_{k_{z}}^{0}:=\hat{\sigma}_{k_{z}}^{0}$ for $z=1,2, \ldots, Z$ except for $z=\arg \min _{z}\left\{\operatorname{cost}\left(\hat{\sigma}_{k_{z}}^{0}\right)-\operatorname{cost}\left(\sigma_{k_{z}}^{0}\right)\right\}$. This customer deletion step is applied to all duplicate customers in a random order. After this procedure, no customer is duplicate, but some customers may remain unassigned. For all of the unassigned customers, the following customer insertion steps are applied. Let $i$ be such a customer, and $j$ be the customer nlist ${ }_{i}^{\phi}[l]$ that has the smallest $l$ among those already assigned to $\boldsymbol{\sigma}^{0}$, where $\phi$ is randomly chosen from $\left\{1,2, \ldots, \nu^{\text {nlist }}\right\}$. Then, customer $i$ is inserted between $j$ and its preceding customer in the current route. The entire procedure of generating an initial solution from $P$, called GENINIT $(P)$, is summarized as follows.

\section{Procedure GENINIT $(P)$}

Step 1 (initialization) Let all routes in $\boldsymbol{\sigma}^{0}$ be empty and let $k:=1$.

Step 2 (routes selection) Let $\sigma^{*}:=\arg \min _{\sigma \in R[P]} c_{\text {gen }}\left(\sigma, \sigma^{0}\right)$. Then let $\sigma_{k}^{0}:=\sigma^{*}$ and $k:=k+1$. If $k \leq m$, return to Step 2 . 
Step 3 (customer deletion) Execute the customer deletion step on duplicate customers until there is no such customers.

Step 4 (customer insertion) Execute the customer insertion step on unassigned customers until there is no such customers.

Step 5 Output $\boldsymbol{\sigma}^{0}=\left(\sigma_{1}^{0}, \sigma_{2}^{0}, \ldots, \sigma_{m}^{0}\right)$ as the initial solution and halt.

Initially, $P$ is set empty. If $|P|<\nu_{\text {pop }}^{\mathrm{AMLS}}$ holds, an initial solution for LS is generated randomly. Then, for the locally optimal solution $\boldsymbol{\sigma}^{\text {lopt }}$ obtained by the LS, we let $P:=P \cup\left\{\boldsymbol{\sigma}^{\text {lopt }}\right\}$ if $\operatorname{cost}\left(\boldsymbol{\sigma}^{\text {lopt }}\right)$ differs from the costs of all solutions in $P$ ( $P$ remains unchanged otherwise). If $|P|=\nu_{\mathrm{pop}}^{\mathrm{AMLS}}$ holds, an initial solution for LS is generated by calling $\operatorname{GENINIT}(P)$. Then for the locally optimal solution $\boldsymbol{\sigma}^{\text {lopt }}$ obtained by the LS, $P$ is updated as follows. Let $\boldsymbol{\sigma}^{\text {worst }}$ be the solution with the worst cost in $P$. If $\operatorname{cost}\left(\boldsymbol{\sigma}^{\text {lopt }}\right)>\operatorname{cost}\left(\boldsymbol{\sigma}^{\text {worst }}\right)$ holds, $P$ remains unchanged. Otherwise, solution $\boldsymbol{\sigma}^{\text {lopt }}$ is added to $P$ and some solutions are deleted from $P$ by the following rules. For this, we define

$$
\operatorname{RCLOSE}\left(\sigma, \sigma^{\prime}\right)=\frac{\left|V_{\sigma} \cap V_{\sigma^{\prime}}\right|}{\left|V_{\sigma} \cup V_{\sigma^{\prime}}\right|}
$$

for a pair of routes $\sigma$ and $\sigma^{\prime}$, which measures the closeness of the assigned customers between the two routes. $\operatorname{RcLose}\left(\sigma, \sigma^{\prime}\right)=1$ holds if and only if the assigned customers of $\sigma$ and $\sigma^{\prime}$ are exactly the same. For a pair of solutions $\boldsymbol{\sigma}=\left(\sigma_{1}, \sigma_{2}, \ldots, \sigma_{m}\right)$ and $\boldsymbol{\sigma}^{\prime}=\left(\sigma_{1}^{\prime}, \sigma_{2}^{\prime}, \ldots, \sigma_{m}^{\prime}\right)$, let

$$
\operatorname{SCLOSE}\left(\boldsymbol{\sigma}, \boldsymbol{\sigma}^{\prime}\right)=\frac{1}{m} \sum_{k=1}^{m} \max _{k^{\prime}}\left\{\operatorname{RCLOSE}\left(\sigma_{k}, \sigma_{k^{\prime}}\right)\right\}
$$

which measures the closeness of the assigned customers between the two solutions. $\operatorname{SCLOSE}\left(\boldsymbol{\sigma}, \boldsymbol{\sigma}^{\prime}\right)=$ 1 holds if and only if the assigned customers are exactly the same. Let $\bar{m}$ (resp., $\bar{s}$ ) be the mean (resp., standard deviation) of $\operatorname{SCLOSE}\left(\boldsymbol{\sigma}, \boldsymbol{\sigma}^{\prime}\right)$ between all pairs $\left(\boldsymbol{\sigma}, \boldsymbol{\sigma}^{\prime}\right)$ of solutions in $P$, and let $\xi(P)=\bar{m}+2 \bar{s}$ and

$$
S=\left\{\boldsymbol{\sigma} \in P \mid \operatorname{SCLOSE}\left(\boldsymbol{\sigma}^{\mathrm{lopt}}, \boldsymbol{\sigma}\right) \geq \xi(P)\right\} .
$$

$S$ is the set of solutions in $P$ whose assigned customers are similar to that of $\boldsymbol{\sigma}^{\text {lopt }}$. If $S=\emptyset$ holds, we let $P:=P \cup\left\{\boldsymbol{\sigma}^{\text {lopt }}\right\} \backslash\left\{\boldsymbol{\sigma}^{\text {worst }}\right\}$. Otherwise (i.e., $S \neq \emptyset$ ), we let $S^{\prime}:=\{\boldsymbol{\sigma} \in S \mid \operatorname{cost}(\boldsymbol{\sigma}) \geq$ $\left.\operatorname{cost}\left(\boldsymbol{\sigma}^{\text {lopt }}\right)\right\}$ and then let $P:=P \cup\left\{\boldsymbol{\sigma}^{\text {lopt }}\right\} \backslash S^{\prime}$ if $S^{\prime} \neq \emptyset\left(P\right.$ remains unchanged if $\left.S^{\prime}=\emptyset\right)$. The above rules of updating set $P$ for the obtained locally optimal solution $\boldsymbol{\sigma}^{\text {lopt }}$ is summarized as follows.

Procedure UPDATE $\left(P, \boldsymbol{\sigma}^{\mathrm{lopt}}\right)$

Step 1 If $|P|<\nu_{\mathrm{pop}}^{\mathrm{AMLS}}$ and $\operatorname{cost}\left(\boldsymbol{\sigma}^{\mathrm{lopt}}\right)$ differs from the costs of all solutions in $P$, let $P:=$ $P \cup\left\{\boldsymbol{\sigma}^{\text {lopt }}\right\}$ and halt.

Step $2\left(|P|=\nu_{\text {pop }}^{\text {AMLS }}\right.$ holds. $) \quad$ For the worst solution $\boldsymbol{\sigma}^{\text {worst }}$ in $P$, halt if $\operatorname{cost}\left(\boldsymbol{\sigma}^{\text {lopt }}\right)>$ $\operatorname{cost}\left(\boldsymbol{\sigma}^{\text {worst }}\right)$ holds. 
Step 3 Let $S:=\left\{\boldsymbol{\sigma} \in P \mid \operatorname{SCLOSE}\left(\boldsymbol{\sigma}^{\text {lopt }}, \boldsymbol{\sigma}\right) \geq \xi(P)\right\}$. If $S=\emptyset$, let $P:=P \cup\left\{\boldsymbol{\sigma}^{\text {lopt }}\right\} \backslash\left\{\boldsymbol{\sigma}^{\text {worst }}\right\}$. Otherwise, compute $S^{\prime}:=\left\{\boldsymbol{\sigma} \in S \mid \operatorname{cost}(\boldsymbol{\sigma}) \geq \operatorname{cost}\left(\boldsymbol{\sigma}^{\text {lopt }}\right)\right\}$, and let $P:=P \cup\left\{\boldsymbol{\sigma}^{\text {lopt }}\right\} \backslash S^{\prime}$ if $S^{\prime} \neq \emptyset$.

Then algorithm AMLS is summarized as follows.

\section{Algorithm AMLS}

Step 1 Let best $:=\infty$ and $P:=\emptyset$.

Step 2 If $|P|<\nu_{\text {pop }}^{\text {AMLS }}$, generate an initial solution $\boldsymbol{\sigma}^{0}$ randomly. Otherwise, let $\boldsymbol{\sigma}^{0}:=$ $\operatorname{GENINIT}(P)$.

Step 3 Improve $\boldsymbol{\sigma}^{0}$ by LS; i.e., let $\boldsymbol{\sigma}^{\text {lopt }}:=\operatorname{LS}\left(\boldsymbol{\sigma}^{0}\right)$.

Step 4 If a solution $\boldsymbol{\sigma}^{\prime}$ satisfying besteval $\left(\boldsymbol{\sigma}^{\prime}\right)<$ best is found during the execution of LS, let best $:=\operatorname{besteval}\left(\boldsymbol{\sigma}^{\prime}\right)$ and $\boldsymbol{\sigma}^{\text {best }}:=\boldsymbol{\sigma}^{\prime}$.

Step 5 Update set $P$ by calling $\operatorname{UPDATE}\left(P, \boldsymbol{\sigma}^{\text {lopt }}\right)$.

Step 6 If some stopping criterion is satisfied, output $\sigma^{\text {best }}$ and halt; otherwise return to Step 2.

\section{Computational results}

We conducted various computational experiments to evaluate the proposed algorithms. The algorithms were coded in $\mathrm{C}$ language and run on a handmade PC (Intel Pentium III $1 \mathrm{GHz}, 1$ GB memory). The program parameters were set as shown in Table 1 unless otherwise stated. The computational time was measured by using the subroutine available at Tsuchimura's web site (http://www.nn.iij4u.or.jp/ tutimura/c/cpu_time.c).

\subsection{The vehicle routing problem with hard time windows}

\subsubsection{Solomon's benchmark instances}

The benchmark instances by Solomon (1987) are widely used in the literature. The number of customers in each instance is 100 , and their locations are distributed in the square $[0,100]^{2}$ of the plane. The distances between customers are measured by Euclidean distance (in double precision), and the traveling times are proportional to the corresponding distances. Each customer $i$ (including the depot) has one time window $\left[w_{i}^{\mathrm{r}}, w_{i}^{\mathrm{d}}\right]$, an amount of requirement $q_{i}$ and a service time $u_{i}$. All vehicles $k$ have a fixed capacity $Q$. Both time window and capacity constraints are considered hard. For these instances, the number of vehicles $m$ is also a decision variable, and the objective is to find a solution with the minimum $\left(m, d_{\text {sum }}(\boldsymbol{\sigma})\right)$ in the lexicographical order. (For two vectors $\alpha=\left(\alpha_{1}, \alpha_{2}, \ldots, \alpha_{l}\right)$ and $\beta=\left(\beta_{1}, \beta_{2}, \ldots, \beta_{l}\right), \alpha$ is smaller than $\beta$ in the lexicographical order if and only if there is an index $i \in[1, l]$ that satisfies $\alpha_{j}=\beta_{j}$ for all $j<i$ and $\alpha_{i}<\beta_{i}$.) These benchmark instances consist of six different sets of problem instances called 
R1, R2, RC1, RC2, C1 and C2, respectively. Locations of customers are uniformly distributed in type $\mathrm{R}$ and are clustered in groups in type $\mathrm{C}$, and the two types are mixed in type $\mathrm{RC}$. Furthermore, for instances of type 1, the time window is narrow at the depot, and hence only a small number of customers can be served by one vehicle. Conversely, for instances of type 2, the time window is wide, and hence many customers can be served by one vehicle.

\subsubsection{Computational results}

As Solomon's benchmark instances are constructed for VRPHTW, we use the lexicographical order of the vector $\left(q_{\text {sum }}(\boldsymbol{\sigma}), p_{\text {sum }}^{*}(\boldsymbol{\sigma}), d_{\text {sum }}(\boldsymbol{\sigma})\right)$ as besteval $(\boldsymbol{\sigma})$ in Section 3.2. As the penalty function $p_{i}(t)$ for customer $i$, we use formula (6). How we set the number of vehicles $m$ and parameter $\alpha$ in (6) is described in Appendix.

We first tested algorithm MLS without incorporating $N^{\text {cyclic }}$ to see the effectiveness of the time-oriented neighbor-lists. Recall that the time-oriented neighbor-lists are used only for $N^{20 p t *}$ and $N^{\text {cross }}$. Neighborhood $N^{\text {intra }}$ is also used in this experiment, since the solution quality is poor without $N^{\text {intra }}$, and its computational time is small compared to that of $N^{\text {cross }}$. We tested two values $L_{\text {cross }}^{\text {nlist }}=30,100\left(L_{\text {cross }}^{\text {nlist }}=100\right.$ means that the whole neighborhood $N^{\text {cross }} \cup N^{2 o p t *}$ is used). The value of $L_{2 \mathrm{opt} *}^{\text {nlist }}$ is set to $\min \left\{\left(L^{\text {cross }}\right)^{2} L_{\text {cross }}^{\text {nlist }}, n\right\}$ as discussed in Section 4.3. Algorithm LS was repeated from randomly generated initial solutions 50 times for each value of $L_{\text {cross }}^{\text {nlist }}$, and the average values of the cost and the computational time were compared. Though the results are omitted due to space limitation, it was observed that, if $L_{\text {cross }}^{\text {nlist }}$ is set to 30 , the average computational time is reduced to $20-30 \%$ of those with $L_{\text {cross }}^{\text {nlist }}=100$, while maintaining almost equivalent solution quality. Actually, LS with $L_{\text {cross }}^{\text {nlist }}=30$ obtained better average cost for some instances.

We then tested the effectiveness of the cyclic exchange neighborhood. For this, we compared the quality of solutions obtained by ILS with and without $N^{\text {cyclic }}$. Both algorithms were run 1800 seconds for each instance, and the solution quality and the number of calls to LS were compared. Though the results are also omitted due to space limitation, not much difference in solution quality was observed. One of the conceivable reasons for this is that the number of calls to LS in ILS without $N^{\text {cyclic }}$ is much larger than that of ILS with $N^{\text {cyclic }}$ (i.e., many LS can compensate the power of the cyclic exchange neighborhood). However, this does not immediately mean that the cyclic exchange neighborhood is useless, as will be observed in Section 6.2.

We finally compare the best solutions obtained by algorithms ILS and AMLS with other existing methods. (The results of MLS are much worse than those of ILS and AMLS, and are omitted.) Both ILS and AMLS were run up to 15000 seconds for each instance. The results are shown in Table 2. "MNV" represents the mean number of vehicles, "MTD" represents the mean (Table 2) total distance, "CNV" represents the cumulative number of vehicles, and "CTD" represents the 
cumulative total distance, which are usually used in the literature to compare the results on Solomon's instances. Column "ILS 2000s" is the result of ILS, where the time limit for each instance is 2000 seconds. The meaning of columns ILS 15000s, AMLS 2000s and AMLS 15000s are similar. Column "H\&G (1999)" is the result by Homberger and Gehring (1999), "G\&H (2002)" is the result by algorithm HM4C in Gehring and Homberger (2002), "BBB (2003)" is the result by Berger, Barkaoui and Bräysy (2003), " $\mathrm{Br}$ (2003)" is the result by algorithm RVNS(2) in Bräysy (2003), "BVH (2001)" is the result by Bent and Van Hentenryck (2001), and "H\&G (2003)" is the result by Homberger and Gehring (2003). According to a recent survey by Bräysy and Gendreau (2003b), not many algorithms achieved CNV 406 or less, and only those algorithms cited in Table 2 achieved CNV 405. The average computational time of algorithms H\&G (1999), G\&H (2002), BBB (2003), Br (2003), and BVH (2001) for each instance are roughly estimated as $800,6000,1300,500$, and 14000 seconds, respectively, if they were run on our computer (see Appendix for the comparison of different computers). Computational time of H\&G (2003) is not clearly stated in Homberger and Gehring (2003). The solution quality of ILS and AMLS with the time limit of 2000 seconds are competitive with H\&G (1999) and G\&H (2002), but slightly worse than BBB (2003), Br (2003), BVH (2001), and H\&G (2003). If much longer computational time, 15000 seconds, is allowed, both ILS and AMLS exhibit better quality than BBB (2003), Br (2003), and BVH (2001). Note that the computational time of our algorithms is roughly equivalent with algorithm BVH (2001).

These results are significant, since our algorithms are very general and not tailored to VRPHTW. More details of the computational results on Solomon's instances are presented in Appendix.

\subsection{Parallel machine scheduling problem}

\subsubsection{The problem definition}

For the parallel machine scheduling problem (PMP), we are given $n$ jobs $\{1,2, \ldots, n\}$ and $m$ identical machines $\{1,2, \ldots, m\}$. A machine can process only one job at a time, and processing of a job can not be stopped, once it begins, until it is completed (i.e., no preemption allowed). Each job $i$ has:

- a processing time $u_{i}(\geq 0)$, and

- a penalty function $p_{i}\left(s_{i}\right)(\geq 0)$ of the start time $s_{i}$ of job $i$.

We are also given a penalty function $p_{0}(t)$ of the completion time $t$ of all jobs in each machine. The objective is to find an assignment of jobs to machines, start times $s_{i}$ of jobs $i(i=1,2, \ldots, n)$ and completion times $s_{k}^{\mathrm{a}}$ of machines $k(k=1,2, \ldots, m)$ so that the following total penalty is 
minimized:

$$
\sum_{i=1}^{n} p_{i}\left(s_{i}\right)+\sum_{k=1}^{m} p_{0}\left(s_{k}^{\mathrm{a}}\right) .
$$

As can be easily understood, this is a special case of VRPGTW in which the distance and traveling time between customers, and the quantity of goods to be delivered to customers are always 0 . We considered this problem in order to observe the performance of our algorithm in dealing with general time penalty functions by generating instances whose optimal values are known.

\subsubsection{Generation of instances}

We generate three instances of PMP, which we call LINEAR, NCONV1 and NCONV2. For each instance, 100 jobs with $u_{i}=10(i=1,2, \ldots, 100)$ are scheduled to 10 machines, and $p_{0}(t)$ is defined as

$$
p_{0}(t)=\max \{-t, 0, t-110\} .
$$

We define $p_{i}(t)$ for $i=1,2, \ldots, 100$ so that $p_{i}(i)=0$ holds for all $i$ in all instances (concrete definition of $p_{i}(t)$ depends on the instance, and will be given later). We define the sets of jobs $I_{k}=\{i \mid i \equiv k-1(\bmod 10)\}, k=1,2, \ldots, 10$. Then, an optimal schedule of cost 0 can be obtained by the following rules.

1. All jobs $i \in I_{k}$ are assigned to machine $k$ for $k=1,2, \ldots, 10$.

2. Start time $s_{i}$ of job $i$ is set to $i$.

3. Completion time $s_{k}^{\mathrm{a}}$ is the completion time of the last job processed on machine $k$.

The three instances differ only in their penalty functions $p_{i}(t)$, which are defined as follows (see Fig. 11).

LINEAR: Penalty functions of $i=1,2, \ldots, 100$ are defined by

$$
p_{i}(t)=\max \{i-t, t-i\} .
$$

NCONV1: Penalty functions of $i=1,2, \ldots, 100$ are defined by

$$
p_{i}(t)= \begin{cases}i-2-t, & t<i-3, \\ t-i+4, & i-3 \leq t<i-2, \\ i-t, & -2 \leq t<i, \\ t-i, & i \leq t<i+2, \\ i+4-t, & i+2 \leq t<i+3, \\ t-i-2, & i+3 \leq t .\end{cases}
$$

NCONV2: There are two types, f-type and b-type, of penalty functions for NCONV2. We denote penalty functions of f-type and b-type for job $i$ as $p_{i}^{\mathrm{f}}(t)$ and $p_{i}^{\mathrm{b}}(t)$, respectively. These 
functions are defined by

$$
p_{i}^{\mathrm{f}}(t)= \begin{cases}i-7-t, & t<i-7 \\ t-i+7, & i-7 \leq t<i-3.5 \\ i-t, & i-3.5 \leq t<i \\ t-i, & i \leq t\end{cases}
$$

and

$$
p_{i}^{\mathrm{b}}(t)= \begin{cases}i-t, & t<i, \\ t-i, & i \leq t<i+3.5 \\ i+7-t, & i+3.5 \leq t<i+7, \\ t-i-7, & i+7 \leq t .\end{cases}
$$

Then, we set $p_{i}(t)=p_{i}^{\mathrm{b}}(t)$ for $1 \leq i \leq 10, p_{i}(t)=p_{i}^{\mathrm{f}}(t)$ for $91 \leq i \leq 100$, and we randomly choose $p_{i}^{\mathrm{f}}(t)$ or $p_{i}^{\mathrm{b}}(t)$ as $p_{i}(t)$ for $11 \leq i \leq 90$.

\subsubsection{Computational results}

Let $\mathrm{MLS}^{-}, \mathrm{ILS}^{-}$and $\mathrm{AMLS}^{-}$be algorithms MLS, ILS and AMLS, respectively, in which $N^{\text {cyclic }}$ is not incorporated. We tested six algorithms, MLS, MLS ${ }^{-}$, ILS, ILS ${ }^{-}$AMLS and AMLS ${ }^{-}$. Each algorithm was run three times for each instance, where each run was terminated after 1800 seconds. Table 3 shows the best costs, average costs and the average number of calls to algorithm LS. It can be observed from the table that MLS, ILS and AMLS are much more powerful than MLS $^{-}$, ILS $^{-}$and AMLS $^{-}$, indicating the effectiveness of the cyclic exchange neighborhood. It can also observed that ILS produces slightly better solutions than MLS and AMLS. In summary, incorporating the cyclic exchange neighborhood is useful for these instances.

\subsection{A production scheduling problem with inventory cost}

\subsubsection{The problem definition}

We consider the production of $\tilde{n}$ items in a given period $[0, X]$ on $m$ identical machines. Each item $i=1,2, \ldots, \tilde{n}$ has

1. a demand $Z_{i}$,

2. an inventory cost coefficient $c_{i}^{\mathrm{I}}$,

3. a production rate $R_{i}$, and each ordered pair of $(i, j)$ for $i, j \in\{1,2, \ldots, \tilde{n}\}$ has

1. a setup cost $c_{i j}^{\mathrm{S}}$,

2. a setup time $t_{i j}^{\mathrm{S}}$,

where $Z_{i}$ is the amount of item $i$ to produce during $[0, X], c_{i}^{\mathrm{I}}$ is the cost incurred for a unit of the accumulated inventory of item $i, R_{i}$ is the time required to produce one unit of item $i, c_{i j}^{\mathrm{S}}$ is the 
cost incurred whenever the production on a machine is changed from item $i$ to item $j\left(c_{i i}^{\mathrm{S}}=0\right)$, and $t_{i j}^{\mathrm{S}}$ is the time required for the setup operation $\left(t_{i i}^{\mathrm{S}}=0\right)$. We assume that the amount $Z_{i} / X$ of item $i$ is consumed per unit time. The production of an item can be divided into lots of possibly different sizes. Let $\zeta_{i}$ be the number of lots of item $i, i_{r}\left(r=1,2, \ldots, \zeta_{i}\right)$ be the $r$ th lot of item $i, \theta_{i_{r}}$ be the amount of production of lot $i_{r}$ (i.e., the lot size) and $s_{i_{r}}$ be the start time of lot $i_{r}$. Then $\sum_{r=1}^{\zeta_{i}} \theta_{i_{r}}=Z_{i}$ must hold. Moreover, let $\sigma_{k}=\left(\sigma_{k}(1), \sigma_{k}(2), \ldots, \sigma_{k}\left(n_{k}\right)\right)$ denote the production order on machine $k$, where $\sigma_{k}(h)$ denote the $h$ th lot in $\sigma_{k}$. Then, a schedule $\boldsymbol{\pi}=(\boldsymbol{\zeta}, \boldsymbol{\theta}, \boldsymbol{\sigma}, \boldsymbol{s})$ is determined by:

1. the number of lots $\zeta_{i}$ of each item $i=1,2, \ldots, \tilde{n}$,

2. the size $\theta_{i_{r}}$ of each lot $i_{r}, r=1,2, \ldots, \zeta_{i}, i=1,2, \ldots, \tilde{n}$,

3. a production order $\sigma_{k}$ on machines $k=1,2, \ldots, m$,

4. start time $s_{i_{r}}$ of production of each lot $i_{r}, r=1,2, \ldots, \zeta_{i}, i=1,2, \ldots, \tilde{n}$.

Determining the start times $s_{i_{r}}$ is not trivial, since the machines can have idle time between adjacent lots. Let $a_{i}^{\boldsymbol{\pi}}(x)$ denote the accumulated amount of item $i$ produced before time $x(0 \leq$ $x \leq X)$, which is determined by the schedule $\boldsymbol{\pi}$. Then define

$$
b_{i}^{\boldsymbol{\pi}}=\max \left\{\max _{0 \leq x \leq X}\left\{\left(Z_{i} / X\right) x-a_{i}^{\boldsymbol{\pi}}(x)\right\}, 0\right\},
$$

which is the amount of item $i$ required to be stored at time 0 so that the shortage of item $i$ will not occur during the entire scheduling period. Then $a_{i}^{\boldsymbol{\pi}}(X)=Z_{i}-b_{i}^{\boldsymbol{\pi}}$ must hold. If $b_{i}^{\boldsymbol{\pi}}$ is stored at time 0 , then the accumulated inventory $I_{i}^{\boldsymbol{\pi}}$ of item $i$ over the whole period $[0, X]$ is given by

$$
I_{i}^{\boldsymbol{\pi}}=\int_{0}^{X}\left(a_{i}^{\boldsymbol{\pi}}(x)+b_{i}^{\boldsymbol{\pi}}-\left(Z_{i} / X\right) x\right) d x .
$$

Our objective function to be minimized is the sum of the total setup costs and the total inventory cost:

$$
\sum_{k=1}^{m} \sum_{h=1}^{n_{k}-1} c_{\sigma_{k}(h), \sigma_{k}(h+1)}^{\mathrm{S}}+\sum_{i=1}^{\tilde{n}} c_{i}^{\mathrm{I}} \cdot I_{i}^{\boldsymbol{\pi}}
$$

\subsubsection{Formulation to VRPGTW}

The production scheduling problem with inventory cost can be formulated to VRPGTW as follows. The formulation includes some approximation on lot sizing and inventory cost. First, we regard the number of lots $\zeta_{i}$ as a parameter, and divide the demand $Z_{i}$ into $\zeta_{i}$ lots of the same amount $Z_{i} / \zeta_{i}$. (If $\zeta_{i}$ are set larger, the approximation is more precise, since consecutive production of lots of the same item can be regarded as one lot.) In the VRPGTW formulation, each customer represents the production of a lot, and each vehicle represents a machine. Then, visiting customer $i_{r}$ by vehicle $k$ signifies that the $r$ th lot of item $i$ is produced on machine $k$. The service time $u_{i_{r}}$ of customer $i_{r}$ then becomes $R_{i} Z_{i} / \zeta_{i}$. We let the distance $d_{i_{r} j_{r^{\prime}}}$ from customer 
$i_{r}$ to customer $j_{r^{\prime}}$ be $d_{i_{r} j_{r^{\prime}}}=c_{i j}^{\mathrm{S}}$, and the travel time $t_{i_{r} j_{r^{\prime}}}$ from customer $i_{r}$ to customer $j_{r^{\prime}}$ be $t_{i_{r} j_{r^{\prime}}}=t_{i j}^{\mathrm{S}}$. Finally, we set the time penalty function $p_{i_{r}}(t)$ to represent the inventory cost of item $i$ as follows. Since we divide demand $Z_{i}$ into $\zeta_{i}$ lots, the desirable start time of lot $i_{r}$ is

$$
s_{i_{r}}^{*}=(r-1) X / \zeta_{i}
$$

If the production of lot $i_{r}$ begins earlier than $s_{i_{r}}^{*}$ by one unit time, the accumulated inventory $I_{i}^{\boldsymbol{\pi}}$ increases by $Z_{i} / \zeta_{i}$. Conversely, if the production of lot $i_{r}$ begins later than $s_{i_{r}}^{*}$ by one unit time, $b_{i}^{\boldsymbol{\pi}}$ should be increased to avoid shortage, and we consider that the accumulated inventory $I_{i}^{\boldsymbol{\pi}}$ increases by $Z_{i}$. Based on these observations, we set the time penalty function $p_{i_{r}}(t)$ as

$$
p_{i_{r}}(t)=\max \left\{\alpha_{i_{r}}^{\mathrm{r}}\left(s_{i_{r}}^{*}-x\right), \alpha_{i_{r}}^{\mathrm{d}}\left(x-s_{i_{r}}^{*}\right)\right\},
$$

where $\alpha_{i_{r}}^{\mathrm{r}}=c_{i}^{\mathrm{I}} Z_{i} / \zeta_{i}$ and $\alpha_{i_{r}}^{\mathrm{d}}=c_{i}^{\mathrm{I}} Z_{i}$. (The above penalty becomes larger than the actual inventory cost if more than one lot of one item is late, since the amounts of inventory to be added to $b_{i}^{\pi}$ are summed up in our penalty function, whereas only the maximum of such amounts for each item contributes to the real inventory cost.)

\subsubsection{Computational results}

We conducted computational experiment on real data from Kokuyo Co., Ltd. In the company, the scheduling period $X$ is one month, and one unit of operating time is eight hours. It is requested by the company that the minimum production amount of item $i$ at a time should be the amount to be produced in eight hours. For this, we set parameter $\zeta_{i}$ so that $Z_{i} / \zeta_{i}$ becomes equal to that amount. The number of machines $m$ is 3 , and the total number of lots $n=\sum_{i=1}^{\tilde{n}} \zeta_{i}$ is summarized in Table 4.

(Table 4)

For this problem, we only tested ILS, since the objective of this experiment is to see the wide applicability of our problem formulation. For each instance, ILS was run until the number of calls to LS reaches 300 . Table 4 shows the costs in yen of the schedules obtained by ILS and those of real schedules currently used by the company. (Note that the costs of our algorithm are recomputed after the schedules are obtained; i.e., they are not the approximate values used in the algorithm.) The computational times of ILS in seconds and the reduction ratio of the costs in $\%$ are also shown. Significant reduction in cost can be observed from the table.

\section{Conclusion}

We considered the vehicle routing problem with general time window constraints (VRPGTW), and proposed local search algorithms. Problem VRPGTW is quite general in that time window constraints are represented by general penalty functions (e.g., more than one time window for 
each customer can be treated). We proposed a dynamic programming algorithm to efficiently compute the optimal start times of services for customers in a given route. We also incorporated an advanced neighborhood, the cyclic exchange neighborhood, and the time-oriented neighborlists to make the local search more effective. As for the metaheuristic frameworks of local search, we tested the multi-start local search (MLS), the iterated local search (ILS) and the adaptive multi-start local search (AMLS). The computational results on Solomon's benchmark instances indicated that the proposed algorithms are quite effective. A parallel machine scheduling problem and a production scheduling problem with inventory cost were also solved to show a wide applicability of the proposed formulation of VRPGTW.

\section{Acknowledgments}

The authors are grateful to Akira Tanaka, Kokuyo Co., Ltd., for providing us the problem instances in Section 6.3. They are also grateful to Olli Bräysy, SINTEF Applied Mathematics, Dorit S. Hochbaum, University of California, Jörg Homberger, University of Cooperative Education, and the anonymous referees for valuable comments. This research was partially supported by Scientific Grant-in-Aid, by the Ministry of Education, Culture, Sports, Science and Technology of Japan, and by the Telecommunications Advancement Foundation of Japan.

\section{References}

R. K. Ahuja, O. Ergun, J. B. Orlin, and A. P. Punnen, "A Survey of Very Large-Scale Neighborhood Search Techniques," Discrete Applied Mathematics 123, 75-102 (2002).

R. K. Ahuja and J. B. Orlin, "A Fast Scaling Algorithm for Minimizing Separable Convex Functions Subject to Chain Constraints," Operations Research 49, 784-789 (2001).

R. K. Ahuja, J. B. Orlin, and D. Sharma, "Very Large-Scale Neighborhood Search," International Transactions in Operations Research 7, 301-317 (2000).

R. Bent and P. Van Hentenryck, "A Two-Stage Hybrid Local Search for the Vehicle Routing Problem with Time Windows," Technical Report, CS-01-06, Department of Computer Science, Brown University, Providence, RI 02912, September 2001.

J. Berger, M. Barkaoui, and O. Bräysy, "A Route-Directed Hybrid Genetic Approach for the Vehicle Routing Problem with Time Windows," INFOR, to appear (2003).

D. P. Bertsekas, Nonlinear Programming, Athena Scientific, Belmont (1995).

K. D. Boese, A. B. Kahng, and S. Muddu, "A New Adaptive Multi-Start Technique for Combinatorial Global Optimizations," Operations Research Letters 16, 101-113 (1994). 
O. Bräysy, "A Reactive Variable Neighborhood Search for the Vehicle Routing Problem with Time Windows," INFORMS Journal on Computing, to appear (2003).

O. Bräysy and M. Gendreau "Vehicle Routing Problem with Time Windows, Part I: Route Construction and Local Search Algorithms," Transportation Science, to appear (2003a).

O. Bräysy and M. Gendreau "Vehicle Routing Problem with Time Windows, Part II: Metaheuristics," Transportation Science, to appear (2003b).

V. Chvátal, Linear Programming, Freeman, New York (1983).

J. S. Davis and J. J. Kanet, "Single-Machine Scheduling with Early and Tardy Completion Costs," Naval Research Logistics 40, 85-101 (1993).

C. De Jong, G. Kant, and A. Van Vliet, On Finding Minimal Route Duration in the Vehicle Routing Problem with Multiple Time Windows," Manuscript, Department of Computer Science, $\begin{array}{lllll}\text { Utrecht } & \text { University, } & \text { Holland, } & 1996 & \text { (available from }\end{array}$ http://www.cs.uu.nl/research/projects/alcom/wp4.3.html).

M. Desrochers, J. K. Lenstra, M. W. P. Savelsbergh, and F. Soumis, "Vehicle Routing with Time Windows: Optimization and Approximation," in Vehicle Routing: Methods and Studies, B. L. Golden and A. A. Assad (eds), 65-84, North-Holland, Amsterdam, 1988.

J. Desrosiers, Y. Dumas, M. M. Solomon, and F. Soumis, "Time Constrained Routing and Scheduling," in Handbooks in Operations Research and Management Science, Vol. 8, Network Routing, M. O. Ball, T. L. Magnanti, C. L. Monma, and G. L. Nemhauser (eds), 35-139, North-Holland, Amsterdam, 1995.

J. J. Dongarra, "Performance of Various Computers Using Standard Linear Equations Software," Technical Report No. CS-89-85, Computer Science Department, University of Tennessee, Knoxville, TN 37996-1301, July 29, 2002 (latest version is available as http://www.netlib.org/benchmark/performance.ps).

M. R. Garey and D. S. Johnson, Computers and Intractability: A Guide to the Theory of NPCompleteness, Freeman, New York (1979).

M. R. Garey, R. E. Tarjan, and G. T. Wilfong, "One-Processor Scheduling with Symmetric Earliness and Tardiness Penalties," Mathematics of Operations Research 13, 330-348 (1988).

H. Gehring and J. Homberger, "Parallelization of a Two-Phase Metaheuristic for Routing Problems with Time Windows," Journal of Heuristics 8, 251-276 (2002).

F. Glover, "Ejection Chains, Reference Structures and Alternating Path Methods for Traveling Salesman Problems," Discrete Applied Mathematics 65, 223-253 (1996).

D. S. Hochbaum, "Approximating Covering and Packing Problems: Set Cover, Vertex Cover, In- 
dependent Set, and Related Problems," in Approximation Algorithms for NP-Hard Problems, D. S. Hochbaum (ed), 94-143, PWS Publishing Company, Boston, 1997.

D. S. Hochbaum, "Solving Integer Programs Over Monotone Inequalities in Three Variables: A Framework for Half Integrality and Good Approximations," European Journal of Operational Research 140, 291-321 (2002a).

D. S. Hochbaum, private communication, 2002b.

D. S. Hochbaum and J. Naor, "Simple and Fast Algorithms for Linear and Integer Programs with Two Variables Per Inequality," SIAM Journal on Computing 23, 1179-1192 (1994).

D. S. Hochbaum and M. Queyranne, "Minimizing a Convex Cost Closure Set," SIAM Journal on Discrete Mathematics 16, 192-207 (2003).

J. Homberger and H. Gehring, "Two Evolutionary Metaheuristics for the Vehicle Routing Problem with Time Windows," INFOR 37, 297-318 (1999).

J. Homberger and H. Gehring, "A Two-Phase Hybrid Metaheuristic for the Vehicle Routing Problem with Time Windows," European Journal of Operational Research, to appear (2003).

D. S. Johnson, "Local Optimization and the Traveling Salesman Problem," in Automata, Languages and Programming, Vol. 443 of Lecture Notes in Computer Science, M. S. Paterson (ed), 446-461, Springer-Verlag, Berlin, 1990.

D. S. Johnson and L. A. McGeoch, "The Traveling Salesman Problem: A Case Study," in Local Search in Combinatorial Optimization, E. H. L. Aarts and J. K. Lenstra (eds), 215-310, John Wiley \& Sons, Chichester, 1997.

Y. A. Koskosidis, W. B. Powell, and M. M. Solomon, "An Optimization-Based Heuristic for Vehicle Routing and Scheduling with Soft Time Window Constraints," Transportation Science 26, 69-85 (1992).

S. Lin, "Computer Solutions of the Traveling Salesman Problem," Bell System Technical Journal 44, 2245-2269 (1965).

S. Lin and B. W. Kernighan, "An Effective Heuristic Algorithm for the Traveling Salesman Problem," Operations Research 21, 498-516 (1973).

O. Martin, S. W. Otto, and E. W. Felten, "Large-Step Markov Chains for the Traveling Salesman Problem," Complex Systems 5, 299-326 (1991).

O. Martin, S. W. Otto, and E. W. Felten, "Large-Step Markov Chains for the TSP Incorporating Local Search Heuristic," Operations Research Letters 11, 219-224 (1992).

I. Or, Traveling Salesman-Type Combinatorial Problems and Their Relation to the Logistics of Regional Blood Banking, Ph.D. Thesis, Department of Industrial Engineering and Manage- 
ment Sciences, Northwestern University, Evanston, IL (1976).

N. Park, H. Okano, and H. Imai, "A Path-Exchange-Type Local Search Algorithm for Vehicle Routing and Its Efficient Search Strategy," Journal of the Operations Research Society of Japan 43, 197-208 (2000).

J. Y. Potvin, T. Kervahut, B. L. Garcia, and J. M. Rousseau, "The Vehicle Routing Problem with Time Windows, Part 1: Tabu Search," INFORMS Journal on Computing 8, 158-164 (1996).

S. Reiter and G. Sherman, "Discrete Optimizing," J. Society for Industrial and Applied Mathematics 13, 864-889 (1965).

L.-M. Rousseau, M. Gendreau, and G. Pesant, "Using Constraint-Based Operators to Solve the Vehicle Routing Problem with Time Windows," Journal of Heuristics 8, 43-58 (2002).

M. W. P. Savelsbergh, "The Vehicle Routing Problem with Time Windows: Minimizing Route Duration," ORSA Journal on Computing 4, 146-154 (1992).

M. M. Solomon, "The Vehicle Routing and Scheduling Problems with Time Window Constraints," Operations Research 35, 254-265 (1987).

M. M. Solomon and J. Desrosiers, "Time Window Constrained Routing and Scheduling Problems," Transportation Science 22, 1-13 (1988).

E. Taillard, P. Badeau, M. Gendreau, F. Guertin, and J. Y. Potvin, "A Tabu Search Heuristic for the Vehicle Routing Problem with Soft Time Windows," Transportation Science 31, 170-186 (1997).

H. Tamaki, T. Komori, and S. Abe, "A Heuristic Approach to Parallel Machine Scheduling with Earliness and Tardiness Penalties," Proc. 7th IEEE International Conference on Emerging Technologies and Factory Automation (ETFA'99), pp. 1367-1370, 1999.

H. Tamaki, T. Sugimoto, and M. Araki, "Parallel Machine Scheduling Problem with a NonRegular Objective Function - Minimizing the Sum of Earliness and Tardiness Penalties (in Japanese)," Transactions of the Society of Instrument and Control Engineers 35, 1176-1182 (1999).

S. R. Thangiah, I. H. Osman, and T. Sun, "Hybrid Genetic Algorithm, Simulated Annealing and Tabu Search Methods for Vehicle Routing Problems with Time Windows," Working Paper UKC/IMS/OR94/4, Institute of Mathematics and Statistics, University of Kent, Canterbury, 1994.

P. M. Thompson and J. B. Orlin, "The Theory of Cyclic Transfers," Working Paper OR200-89, Operations Research Center, MIT, Cambridge, MA, 1989. 
P. M. Thompson and H. N. Psaraftis, "Cyclic Transfer Algorithms for Multivehicle Routing and Scheduling Problems," Operations Research 41, 935-946 (1993).

\section{Appendix: Detailed results for Solomon's instances}

The details of the results on Solomon's instances are explained. We will omit the computational results for type $\mathrm{C}$, since these instances are easy and our algorithms always output the best known values (see, e.g., the column "Our best solution" in Tables II and III of Taillard et al. (1997), or Table 8 below) in short time under wide ranges of parameter settings.

The number of vehicles $m$ and parameter $\alpha$ in (6) used in our experiments are listed in Table 5. (As for the instance name, the last two digits represent the instance index and the rest (Table 5) indicates the type. For example, twelve instances R101-R112 belong to type R1.) According to Bräysy (2003), these values of $m$ are taken from the best published solutions except for R101, for which Thangiah, Osman and Sun (1994) reported a solution with one vehicle less. As ILS (resp., AMLS) failed to find a feasible solution with the best known $m$ within 2000 seconds for R104 (resp., RC105), we also ran our algorithms with one vehicle more for these two instances R104 and RC105. Parameter $\alpha$ was chosen after testing 10, 50 and 100 for type 1 instances, and 1, 5 and 10 for type 2 instances, respectively, in preliminary experiments except for RC105 with $m=13$. For RC105 with $m=13$, AMLS failed to find a feasible solution with $\alpha \leq 100$, and we decided to use $\alpha=5000$ after testing $\alpha=500,1000$ and 5000 .

The best solutions obtained by ILS and AMLS are shown in Tables 6 and 7, respectively. The figures in columns "distance" denote the distances $d_{\text {sum }}(\boldsymbol{\sigma})$ of the best solutions $\boldsymbol{\sigma}$ obtained by the algorithms within the time limit shown in the first row. The total number of calls to algorithm LS within the time limit is shown in columns "\#LS in total." Columns "time to best (s)" show the CPU seconds when the best solutions are found. Feasible solutions to VRPHTW (i.e., $p_{\text {sum }}^{*}(\boldsymbol{\sigma})=0$ and $q_{\text {sum }}(\boldsymbol{\sigma})=0$ ) were obtained for all instances except for those with "- " marks. The last two columns labeled "to find feasible" are the number of calls to algorithm LS (column "\#LS to feas.") and CPU seconds (column "time to feas. (s)") when the first feasible solution is found. We compare our results with the best known values reported in http://www.sintef.no/static/am/opti/projects/top/vrp/bknown.html (the data was taken on March 6, 2003), which are summarized in Table 8. A single asterisk "*" indicates a tie with the best known solution and a double asterisk "**" indicates that a better solution was found. ILS obtained three tie solutions, while AMLS obtained one better solution and six tie solutions, among 39 instances in the table.

Finally, we estimate the computational time of algorithms H\&G (1999), G\&H (2002), BBB (2003), Br (2003), and BVH (2001). The benchmark results of SPECint2000, SPECint95 from 
SPEC web page (http://www.specbench.org/) and Dongarra's LINPACK results (Dongarra 2002) of related CPUs are summarized in Table 9. (As we could not find LINPACK results for Pentium $400 \mathrm{MHz}$, Pentium $200 \mathrm{MHz}$, and Sun Ultra $10440 \mathrm{MHz}$ in Dongarra (2002), the corresponding results (i.e., those data in parentheses) were taken from Bräysy and Gendreau (2003b).) We also include Mflops and Mips reported in Gehring and Homberger (2002). As the results of SPECint95 and LINPACK for our CPU (Pentium III $1 \mathrm{GHz}$ ) are not available, the results of similar CPUs, Pentium III $933 \mathrm{MHz}$ and $800 \mathrm{MHz}$, are shown.

Based on these data, we give in Table 10 rough estimates on the computational time spent by algorithms H\&G (1999), G\&H (2002), BBB (2003), Br (2003), and BVH (2001) for types R and RC instances. (The average computational time of Br (2003) reported in Bräysy (2003) on types $\mathrm{R}$ and $\mathrm{RC}$ instances ranges from 129.8 to 288.1 minutes which are the estimated values on Sun Sparc 10 using Dongarra's factors. The computational time of $\mathrm{Br}$ (2003) on Pentium $200 \mathrm{MHz}$ in the table is therefore estimated from these data.) If the results are the best of multiple runs, we multiply the number of runs. Algorithm G\&H (2002) is designed for distributed computation, where 5 computers ( 1 for master and 4 for slaves) were used to obtain the results in Table 2 . In this case, we multiply 4 , the number of slaves, to estimate the time in sequential computation, though this might be an overestimate. 


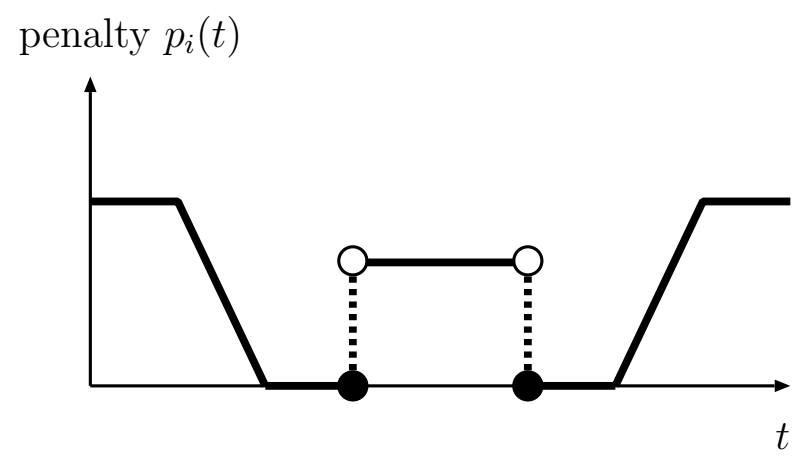

Figure 1. An example of a penalty function $p_{i}(t)$ 


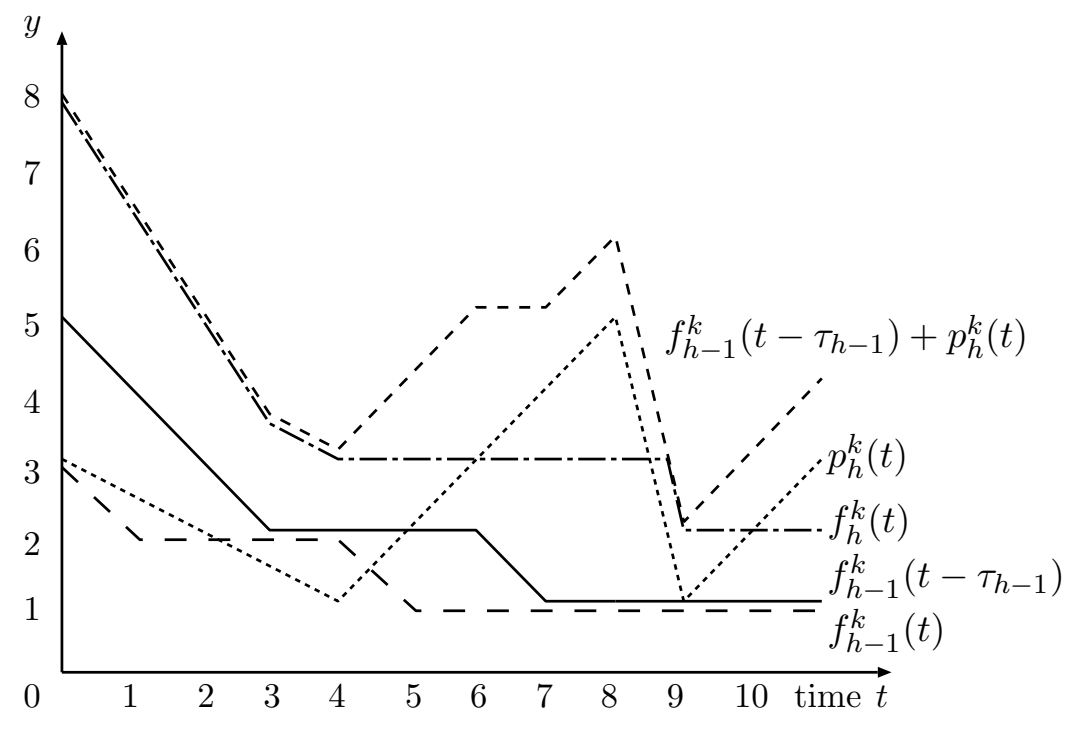

Figure 2. An example of the computation of $f_{h}^{k}(t)$ from $f_{h-1}^{k}(t)$ and $p_{h}^{k}(t)$ 


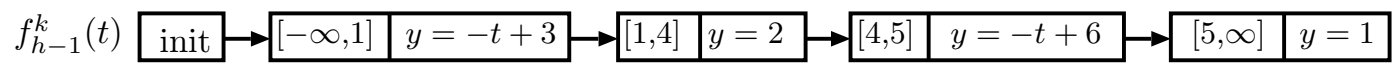

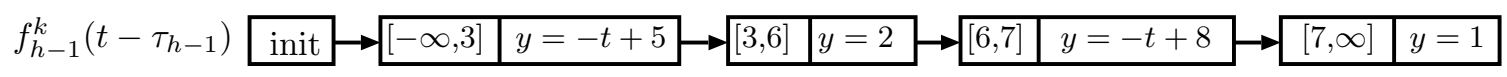

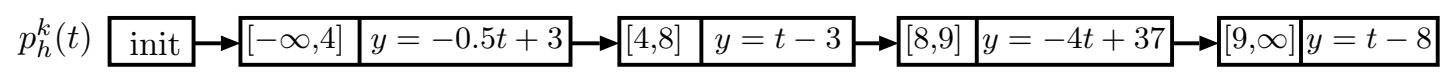

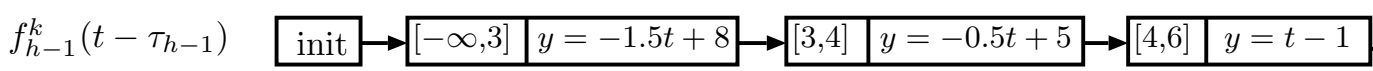

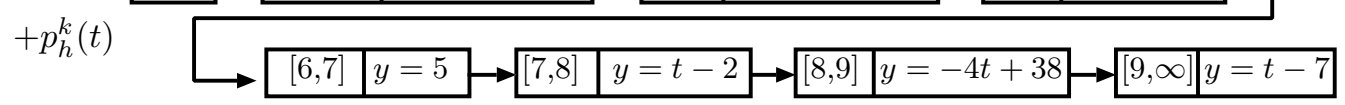

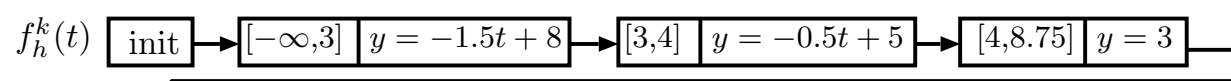

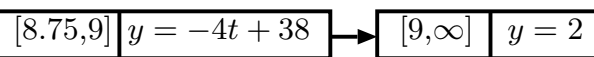

Figure 3. The linked lists representing the 4 functions in Fig. 2 


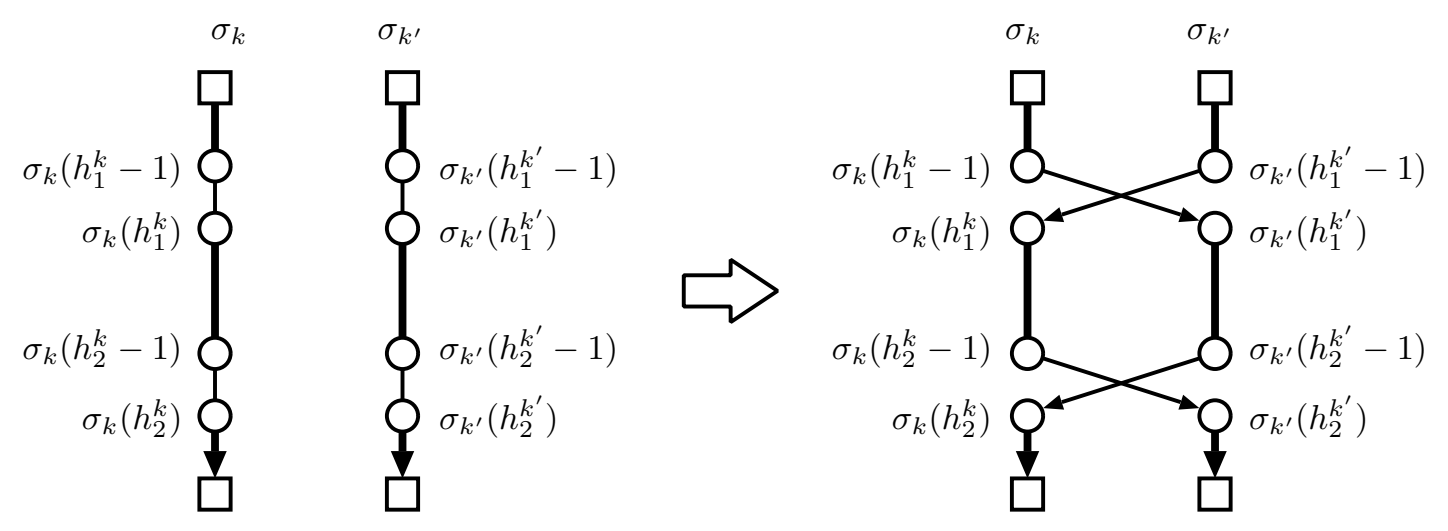

Figure 4. A cross exchange operation 


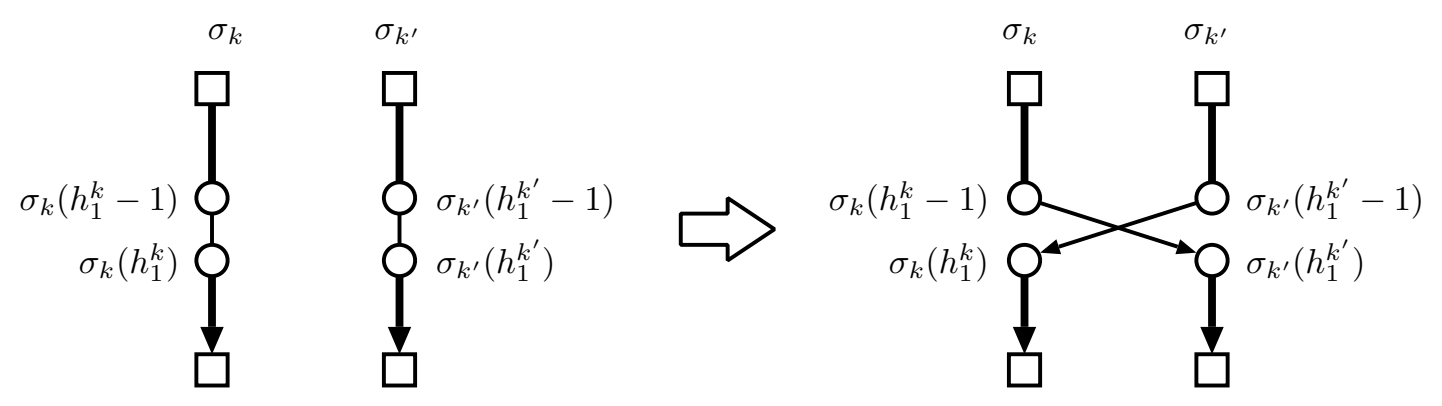

Figure 5. A 2-opt* operation 


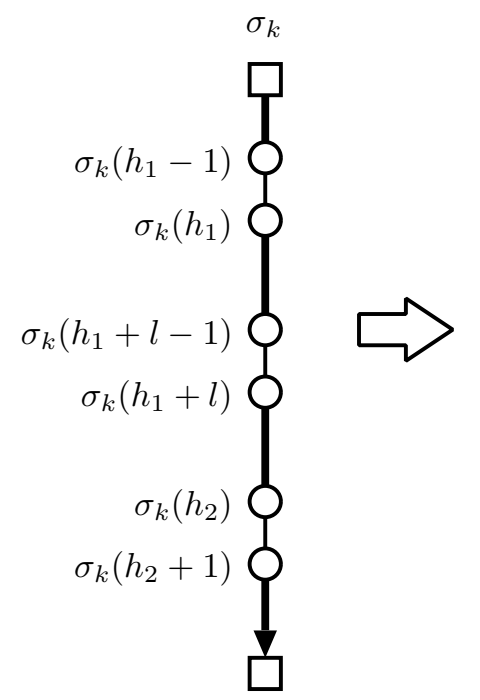

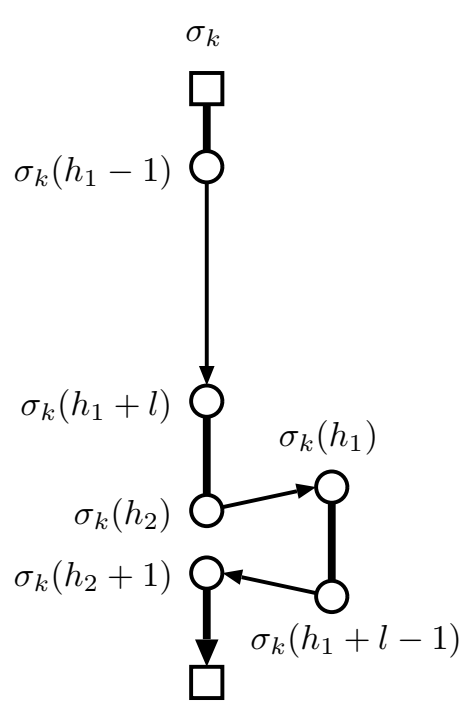

(a) normal insertion

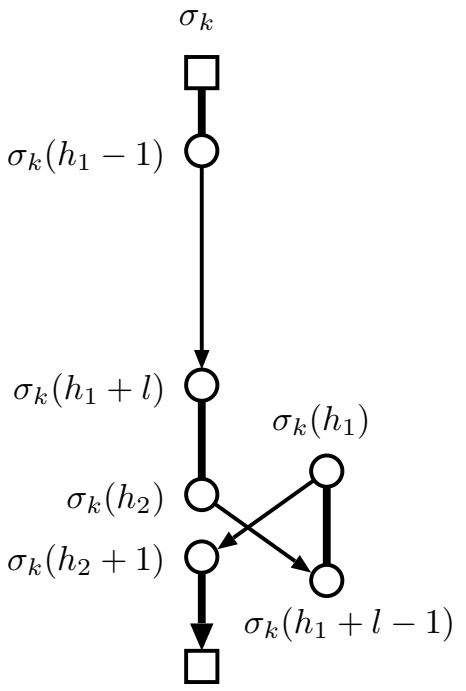

(b) reverse insertion

Figure 6. An intra-route operation: (a) normal and (b) reverse insertions 


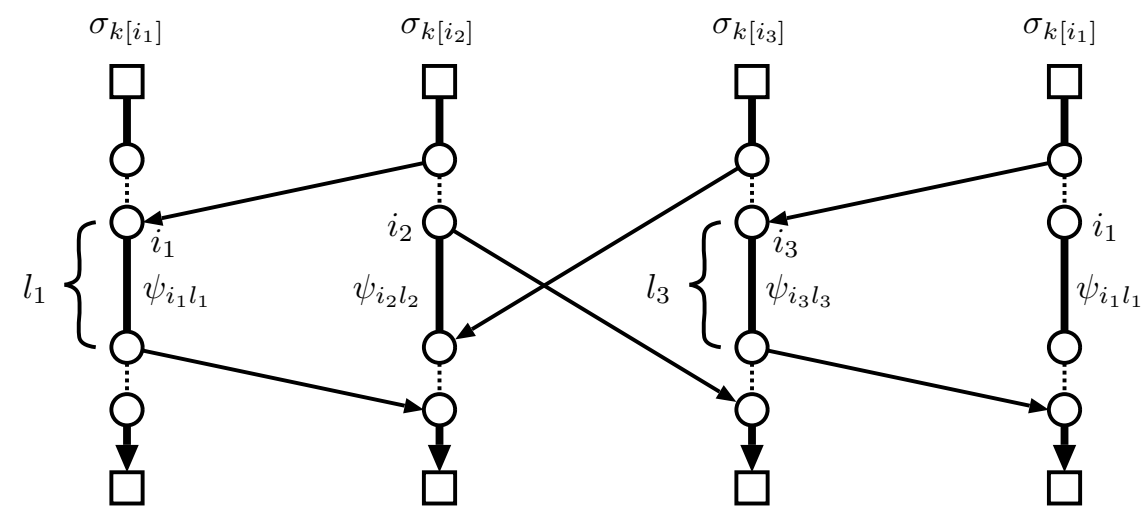

Figure 7. A cyclic exchange operation on three routes (the first and last routes represent the same route) 


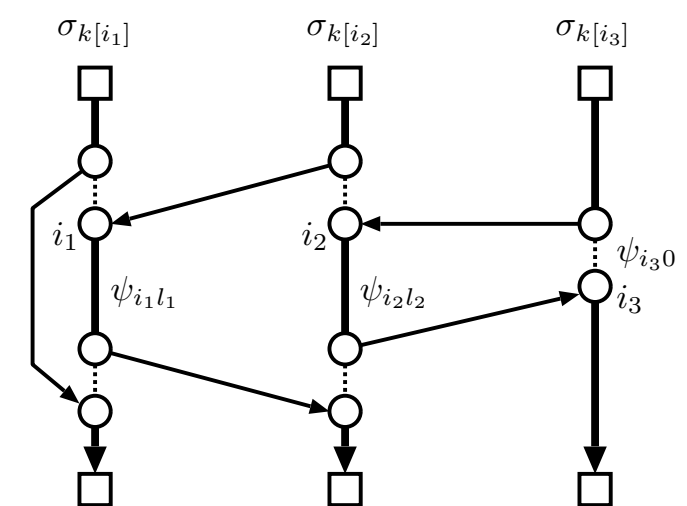

(a) An acyclic exchange on $\psi_{i_{1} l_{1}}, \psi_{i_{2} l_{2}}$ and $\psi_{i_{3} 0}$

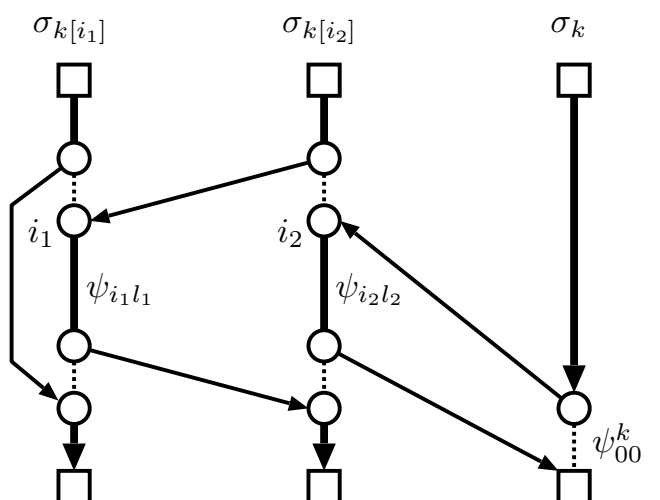

(b) An acyclic exchange on $\psi_{i_{1} l_{1}}, \psi_{i_{2} l_{2}}$ and $\psi_{00}^{k}$

Figure 8. An acyclic exchange operation on three routes 


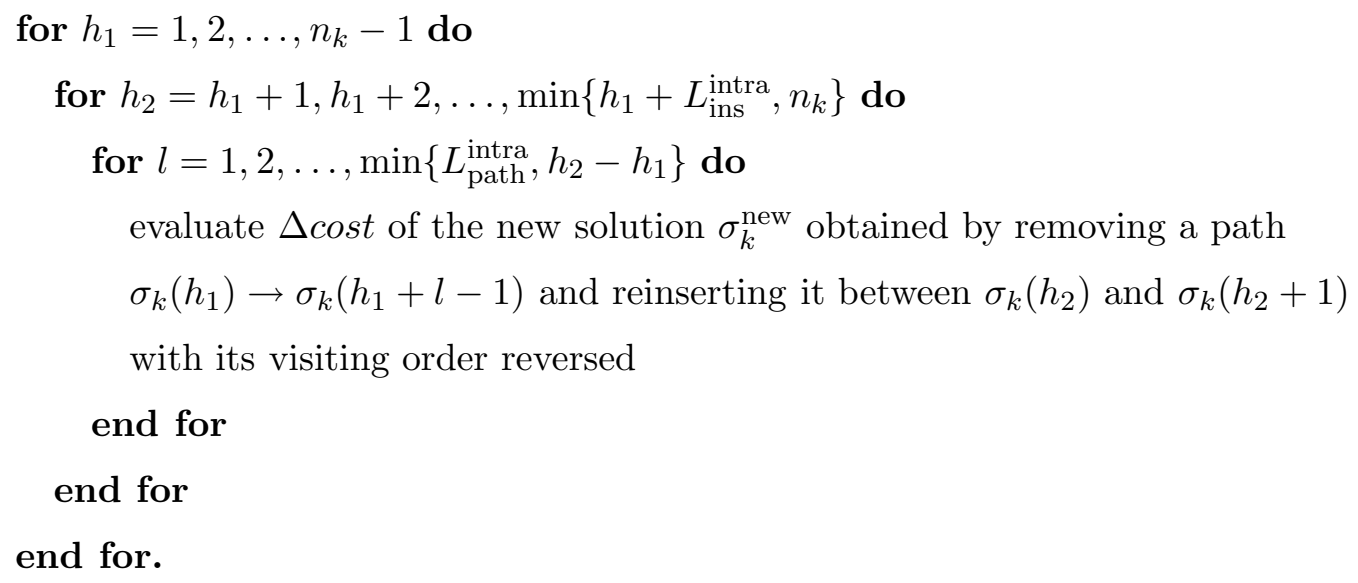

Figure 9. Search order in the intra-route neighborhood with respect to the reverse forward insertion 


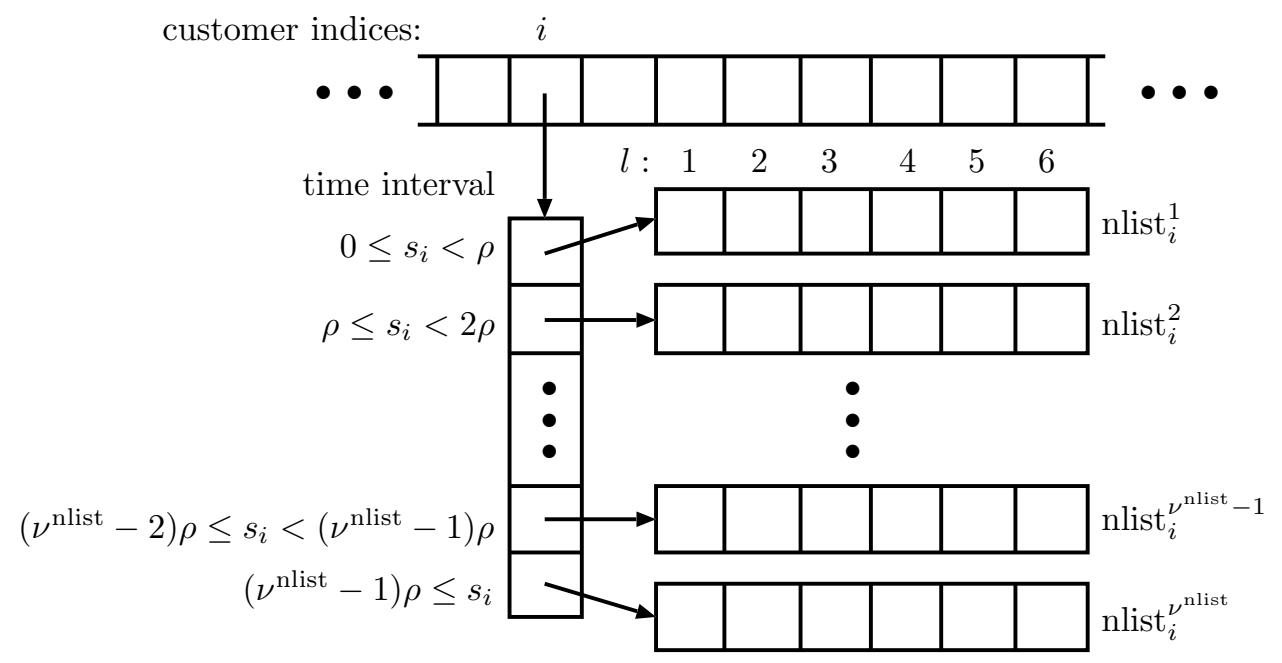

Figure 10. An illustration of the time-oriented neighbor-lists 


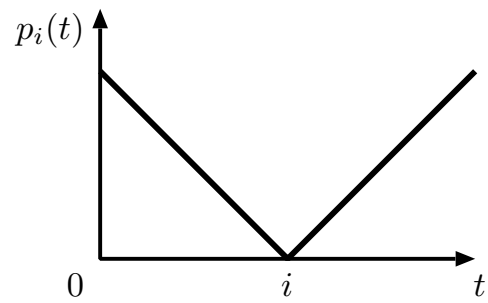

(a) LINEAR

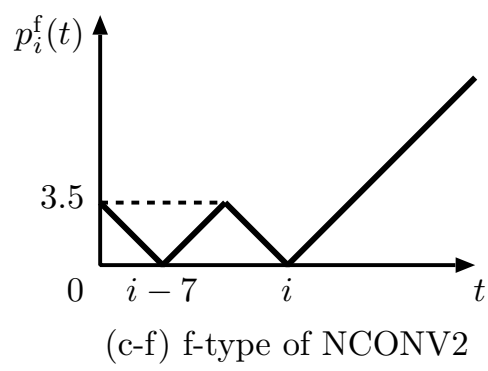

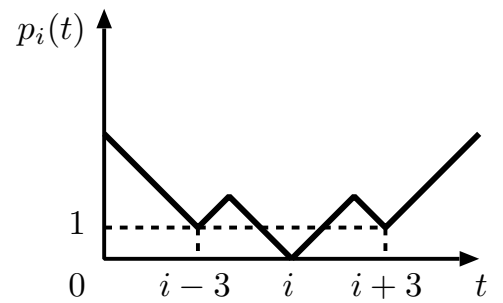

(b) NCONV1

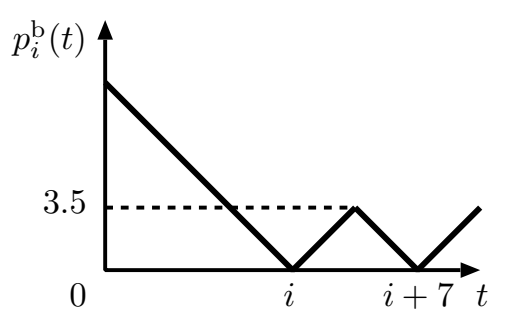

(c-b) b-type of NCONV2

Figure 11. Penalty functions $p_{i}(t)$ : (a) LINEAR, (b) NCONV1, (c-f) f-type of NCONV2 and (c-b) b-type of NCONV2 
Table 1. The default parameter values of our algorithms

\begin{tabular}{ll}
\hline$N^{\text {cross }}:$ & $L^{\text {cross }}=3$ \\
$N^{\text {intra: }}:$ & $L_{\text {path }}^{\text {intra }}=3, L_{\text {ins }}^{\text {intra }}=30$ \\
$N^{\text {cyclic }}:$ & $L^{\text {cyclic }}=3, \nu^{\text {cyclic }}=5$ \\
Neighbor-lists: & $\nu^{\text {nlist }}=20, \mu^{\text {nlist }}=3, L_{\text {cross }}^{\text {nlist }}=20\left(L_{2 \text { opt* }^{\text {nlist }}}^{\text {nling }}\left\{\left(L^{\text {cross }}\right)^{2} L_{\text {cross }}^{\text {nlist }}, n\right\}=100\right)$ \\
ILS: & $\nu_{\text {ptb }}^{\text {ILS }}=1$ \\
AMLS: & $\nu_{\text {pop }}^{\text {AMLS }}=10$ \\
\hline
\end{tabular}


Table 2. Comparison of the solution quality on Solomon's instances

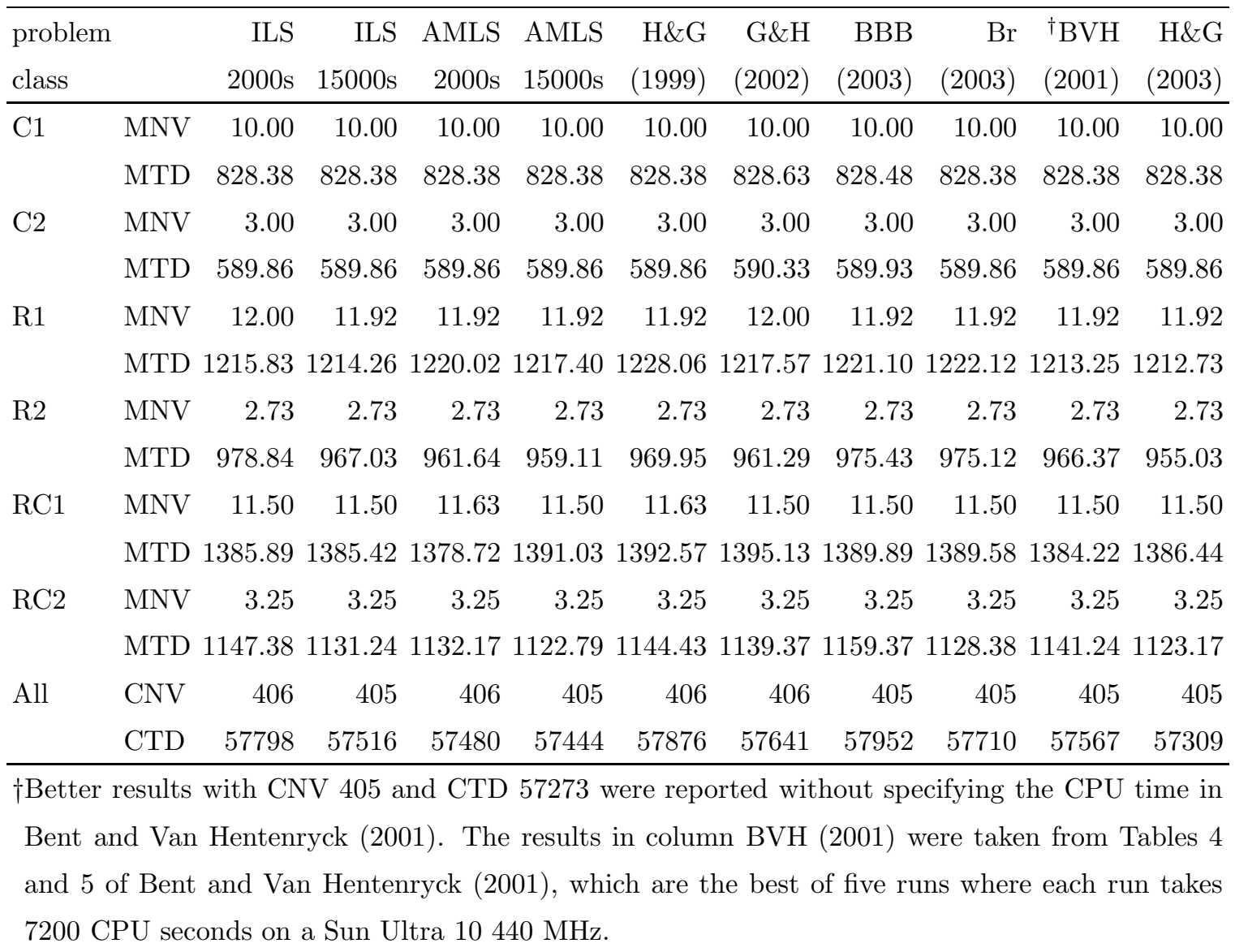


Table 3. The best costs, average costs and the number of calls to algorithm LS by six algorithms applied to the parallel machine scheduling problem

\begin{tabular}{llcccccc}
\hline instance & & MLS & MLS $^{-}$ & ILS & ILS $^{-}$ & AMLS & AMLS $^{-}$ \\
\hline \multirow{2}{*}{ LINEAR } & best cost & 0 & 4 & 0 & 0 & 0 & 2 \\
& avg. cost & 0.0 & 6.7 & 0.0 & 0.7 & 0.0 & 2.0 \\
& \#LS & 101.7 & 1048.0 & 113.7 & 985.7 & 120.0 & 936.3 \\
\hline \multirow{2}{*}{ NCONV1 } & best cost & 0 & 13 & 0 & 0 & 0 & 8 \\
& avg. cost & 0.0 & 15.3 & 0.0 & 1.0 & 0.0 & 9.3 \\
& \#LS & 65.7 & 635.0 & 94.3 & 588.3 & 70.3 & 395.3 \\
\hline \multirow{2}{*}{ NCONV2 } & best cost & 4 & 23 & 0 & 12 & 3 & 21 \\
& avg. cost & 5.3 & 25.0 & 0.0 & 17.7 & 4.0 & 21.3 \\
& \#LS & 55.3 & 720.7 & 94.0 & 677.7 & 58.0 & 403.0 \\
\hline
\end{tabular}


Table 4. Comparison of schedules obtained by ILS and those used in the company

\begin{tabular}{llrrrr}
\hline \multirow{2}{*}{ data } & \multirow{2}{*}{$n$} & cost (yen) of the & \multicolumn{2}{c}{ ILS } & reduction \\
\cline { 4 - 5 } & & real schedule & cost (yen) & CUP secs. & ratio (\%) \\
\hline 1999.11 & 66 & 1443758 & 1094932 & 794 & 24.2 \\
1999.12 & 67 & 1400603 & 919898 & 523 & 34.3 \\
2000.01 & 57 & 1258431 & 913148 & 316 & 27.4 \\
2000.02 & 60 & 1106430 & 758573 & 348 & 31.4 \\
2000.03 & 59 & 1177601 & 858588 & 334 & 27.1 \\
2000.04 & 66 & 1119503 & 698220 & 458 & 37.6 \\
\hline
\end{tabular}


Table 5. The number of vehicles $m$ and parameter $\alpha$ for Solomon's benchmark instances

\begin{tabular}{|c|c|c|c|c|c|c|c|c|c|c|c|}
\hline instance & $m$ & $\alpha$ & instance & $m$ & $\alpha$ & instance & $m$ & $\alpha$ & instance & $m$ & $\alpha$ \\
\hline R101 & 19 & 50 & R201 & 4 & 10 & RC101 & 14 & 100 & $\mathrm{RC} 201$ & 4 & 10 \\
\hline R102 & 17 & 50 & $\mathrm{R} 202$ & 3 & 10 & RC102 & 12 & 100 & $\mathrm{RC} 202$ & 3 & 10 \\
\hline R103 & 13 & 100 & R203 & 3 & 1 & RC103 & 11 & 10 & $\mathrm{RC} 203$ & 3 & 1 \\
\hline R104 & 10 & 10 & R204 & 2 & 10 & RC104 & 10 & 10 & $\mathrm{RC} 204$ & 3 & 1 \\
\hline R104 & 9 & 10 & R205 & 3 & 1 & RC105 & 14 & 100 & RC205 & 4 & 10 \\
\hline R105 & 14 & 10 & R206 & 3 & 1 & $\mathrm{RC} 105$ & 13 & 5000 & $\mathrm{RC} 206$ & 3 & 1 \\
\hline R106 & 12 & 10 & R207 & 2 & 5 & $\mathrm{RC} 106$ & 11 & 100 & $\mathrm{RC} 207$ & 3 & 1 \\
\hline R107 & 10 & 10 & R208 & 2 & 1 & $\mathrm{RC} 107$ & 11 & 10 & RC208 & 3 & 1 \\
\hline R108 & 9 & 10 & R209 & 3 & 1 & RC108 & 10 & 10 & & & \\
\hline R109 & 11 & 50 & $\mathrm{R} 210$ & 3 & 5 & & & & & & \\
\hline R110 & 10 & 50 & R211 & 2 & 10 & & & & & & \\
\hline R111 & 10 & 10 & & & & & & & & & \\
\hline R112 & 9 & 50 & & & & & & & & & \\
\hline
\end{tabular}


Table 6. Detailed results by ILS for Solomon's instances

\begin{tabular}{|c|c|c|c|c|c|c|c|c|c|}
\hline & \multirow[b]{2}{*}{$m$} & \multicolumn{3}{|c|}{ time limit $=2000$ secs } & \multicolumn{3}{|c|}{ time limit $=15000$ secs } & \multicolumn{2}{|c|}{ to find feasible } \\
\hline & & distance & $\begin{array}{r}\text { \#LS in } \\
\text { total }\end{array}$ & $\begin{array}{l}\text { time to } \\
\text { best }(\mathrm{s})\end{array}$ & distance & $\begin{array}{r}\text { \#LS in } \\
\text { total }\end{array}$ & $\begin{array}{l}\text { time to } \\
\text { best }(\mathrm{s})\end{array}$ & $\begin{array}{c}\text { \#LS to } \\
\text { feas. }\end{array}$ & $\begin{array}{l}\text { time to } \\
\text { feas. }(\mathrm{s})\end{array}$ \\
\hline R101 & 19 & 1650.80 & 760 & 190.94 & 1650.80 & 5632 & 190.94 & 21 & 43.01 \\
\hline R102 & 17 & 1487.88 & 598 & 440.49 & 1486.96 & 4073 & 14246.96 & 2 & 5.06 \\
\hline $\mathrm{R} 103$ & 13 & 1297.99 & 775 & 1072.59 & 1293.05 & 5338 & 6343.37 & 96 & 171.39 \\
\hline R104 & 10 & 982.02 & 899 & 1247.93 & 982.02 & 6512 & 1247.93 & 2 & 5.78 \\
\hline $\mathrm{R} 104$ & 9 & - & - & - & 1013.13 & 5313 & 2032.78 & 718 & 2012.69 \\
\hline $\mathrm{R} 105$ & 14 & * 1377.11 & 786 & 486.91 & * 1377.11 & 5319 & 486.91 & 6 & 13.82 \\
\hline $\mathrm{R} 106$ & 12 & 1261.94 & 704 & 736.88 & 1257.96 & 5107 & 9416.67 & 6 & 13.96 \\
\hline R107 & 10 & 1125.40 & 712 & 855.11 & 1120.25 & 5079 & 4869.27 & 92 & 217.28 \\
\hline R108 & 9 & 980.32 & 763 & 994.55 & 962.34 & 5761 & 4013.62 & 49 & 102.82 \\
\hline R109 & 11 & 1197.64 & 761 & 557.19 & * 1194.73 & 5423 & 4700.72 & 92 & 210.70 \\
\hline R110 & 10 & 1119.00 & 763 & 884.47 & 1119.00 & 5499 & 884.47 & 60 & 126.45 \\
\hline R111 & 10 & 1106.30 & 714 & 190.10 & 1101.91 & 5119 & 12034.23 & 25 & 60.61 \\
\hline $\mathrm{R} 112$ & 9 & 1003.55 & 930 & 1984.91 & 993.82 & 6634 & 14486.12 & 817 & 1776.35 \\
\hline $\mathrm{R} 201$ & 4 & 1262.64 & 555 & 1893.11 & 1253.23 & 3805 & 3306.99 & 1 & 3.22 \\
\hline $\mathrm{R} 202$ & 3 & 1201.24 & 338 & 1671.67 & 1200.40 & 2213 & 4555.79 & 5 & 27.13 \\
\hline $\mathrm{R} 203$ & 3 & 953.98 & 313 & 1017.88 & 952.29 & 2208 & 11018.32 & 1 & 7.38 \\
\hline $\mathrm{R} 204$ & 2 & 853.86 & 253 & 200.95 & 853.86 & 1786 & 200.95 & 24 & 164.66 \\
\hline $\mathrm{R} 205$ & 3 & 1026.25 & 308 & 1026.25 & 1009.10 & 2190 & 10856.82 & 1 & 6.10 \\
\hline R206 & 3 & 913.18 & 394 & 1355.17 & 912.01 & 2473 & 9251.25 & 1 & 7.30 \\
\hline $\mathrm{R} 207$ & 2 & 977.54 & 240 & 1616.77 & 898.64 & 1672 & 12453.91 & 82 & 623.46 \\
\hline $\mathrm{R} 208$ & 2 & 736.43 & 278 & 1942.15 & 734.67 & 1787 & 4639.49 & 1 & 8.80 \\
\hline $\mathrm{R} 209$ & 3 & 919.58 & 331 & 944.87 & 919.58 & 2202 & 944.87 & 3 & 14.05 \\
\hline $\mathrm{R} 210$ & 3 & 967.94 & 359 & 526.71 & 962.45 & 2508 & 8211.05 & 1 & 5.49 \\
\hline $\mathrm{R} 211$ & 2 & 954.65 & 248 & 1093.23 & 941.10 & 1678 & 9217.80 & 45 & 314.73 \\
\hline $\mathrm{RC} 101$ & 14 & 1696.95 & 903 & 348.62 & 1696.95 & 6608 & 348.62 & 110 & 215.14 \\
\hline RC102 & 12 & 1554.84 & 873 & 682.01 & 1554.84 & 6150 & 682.01 & 327 & 672.98 \\
\hline RC103 & 11 & 1265.80 & 968 & 471.14 & 1263.54 & 7031 & 5248.24 & 2 & 4.25 \\
\hline $\mathrm{RC} 104$ & 10 & 1137.03 & 978 & 1816.49 & 1136.88 & 6800 & 14816.71 & 13 & 30.49 \\
\hline $\mathrm{RC} 105$ & 14 & 1543.38 & 869 & 1452.53 & 1540.18 & 6165 & 3312.70 & 6 & 14.00 \\
\hline $\mathrm{RC} 105$ & 13 & 1633.72 & 812 & 1550.40 & 1633.72 & 5726 & 1550.40 & 109 & 219.51 \\
\hline $\mathrm{RC} 106$ & 11 & * 1424.73 & 1124 & 573.76 & * 1424.73 & 8039 & 573.76 & 130 & 193.17 \\
\hline $\mathrm{RC} 107$ & 11 & 1232.26 & 1093 & 181.31 & 1232.26 & 7956 & 181.31 & 4 & 6.20 \\
\hline $\mathrm{RC} 108$ & 10 & 1141.76 & 1096 & 700.90 & 1140.40 & 7984 & 7149.54 & 28 & 44.35 \\
\hline $\mathrm{RC} 201$ & 4 & 1423.60 & 602 & 589.51 & 1413.52 & 4011 & 9425.99 & 1 & 2.91 \\
\hline RC202 & 3 & 1459.40 & 344 & 1596.03 & 1388.86 & 2519 & 8374.82 & 42 & 197.19 \\
\hline $\mathrm{RC} 203$ & 3 & 1096.15 & 410 & 1842.24 & 1077.93 & 2642 & 10083.76 & 4 & 16.18 \\
\hline $\mathrm{RC} 204$ & 3 & 799.16 & 458 & 355.70 & 799.16 & 3169 & 355.70 & 1 & 6.32 \\
\hline RC205 & 4 & 1314.40 & 645 & 1337.54 & 1307.15 & 4443 & 10381.77 & 1 & 2.88 \\
\hline $\mathrm{RC} 206$ & 3 & 1167.28 & 378 & 306.16 & 1165.04 & 2553 & 6164.89 & 2 & 10.84 \\
\hline $\mathrm{RC} 207$ & 3 & 1075.92 & 353 & 1118.53 & 1064.05 & 2399 & 2902.25 & 2 & 14.86 \\
\hline RC208 & 3 & 843.15 & 424 & 1936.64 & 834.23 & 2859 & 5116.93 & 1 & 4.58 \\
\hline
\end{tabular}

*: a tie with the best known solution 
Table 7. Detailed results by AMLS for Solomon's instances

\begin{tabular}{|c|c|c|c|c|c|c|c|c|c|}
\hline & \multirow[b]{2}{*}{$m$} & \multicolumn{3}{|c|}{ time limit $=2000$ secs } & \multicolumn{3}{|c|}{ time limit $=15000$ secs } & \multicolumn{2}{|c|}{ to find feasible } \\
\hline & & distance & $\begin{array}{r}\text { \#LS in } \\
\text { total }\end{array}$ & $\begin{array}{l}\text { time to } \\
\text { best }(\mathrm{s})\end{array}$ & distance & $\begin{array}{r}\text { \#LS in } \\
\text { total }\end{array}$ & $\begin{array}{l}\text { time to } \\
\text { best }(\mathrm{s})\end{array}$ & $\begin{array}{c}\text { \#LS to } \\
\text { feas. }\end{array}$ & $\begin{array}{l}\text { time to } \\
\text { feas. (s) }\end{array}$ \\
\hline R101 & 19 & 1651.97 & 909 & 222.56 & 1650.80 & 7217 & 2999.98 & 2 & 7.00 \\
\hline R102 & 17 & 1487.97 & 771 & 180.45 & 1487.97 & 7098 & 180.45 & 5 & 20.88 \\
\hline R103 & 13 & 1293.05 & 952 & 1718.30 & 1293.05 & 8205 & 1718.30 & 61 & 137.66 \\
\hline R104 & 10 & 981.23 & 1268 & 1927.28 & 981.23 & 9922 & 1927.28 & 2 & 6.32 \\
\hline R104 & 9 & 1013.13 & 920 & 1986.56 & 1013.13 & 7718 & 1986.56 & 912 & 1986.56 \\
\hline R105 & 14 & * 1377.11 & 884 & 466.13 & * 1377.11 & 7213 & 466.13 & 5 & 16.58 \\
\hline R106 & 12 & 1258.48 & 852 & 1683.83 & 1257.96 & 7034 & 2915.93 & 13 & 39.10 \\
\hline R107 & 10 & 1120.98 & 1093 & 1165.17 & 1118.04 & 9293 & 5962.73 & 46 & 145.89 \\
\hline R108 & 9 & 977.28 & 922 & 1495.42 & 963.99 & 8699 & 2550.13 & 14 & 39.44 \\
\hline R109 & 11 & 1197.42 & 1215 & 1690.89 & 1197.42 & 10338 & 1690.89 & 29 & 91.40 \\
\hline R110 & 10 & 1142.85 & 1260 & 1650.77 & 1137.10 & 10579 & 2460.66 & 89 & 210.39 \\
\hline R111 & 10 & 1104.54 & 803 & 1913.35 & 1096.73 & 7747 & 4275.11 & 29 & 79.20 \\
\hline R112 & 9 & 1015.50 & 1118 & 1768.87 & 1015.50 & 9168 & 1768.87 & 434 & 903.86 \\
\hline $\mathrm{R} 201$ & 4 & 1256.11 & 1063 & 1900.43 & 1253.23 & 8508 & 2359.64 & 1 & 3.21 \\
\hline $\mathrm{R} 202$ & 3 & 1201.69 & 1044 & 398.19 & 1200.18 & 8326 & 4573.36 & 6 & 31.77 \\
\hline $\mathrm{R} 203$ & 3 & 946.20 & 599 & 1407.59 & 942.86 & 5060 & 13230.44 & 1 & 7.09 \\
\hline R204 & 2 & 848.59 & 678 & 1808.52 & 844.20 & 5740 & 9664.77 & 41 & 259.32 \\
\hline $\mathrm{R} 205$ & 3 & 1012.32 & 519 & 1175.57 & 1004.04 & 4115 & 4749.60 & 1 & 5.95 \\
\hline R206 & 3 & 914.28 & 599 & 1533.23 & 913.26 & 4877 & 13551.43 & 1 & 7.03 \\
\hline $\mathrm{R} 207$ & 2 & 909.80 & 909 & 1574.27 & 908.77 & 7894 & 2601.94 & 54 & 333.98 \\
\hline $\mathrm{R} 208$ & 2 & 728.03 & 601 & 1898.09 & 726.82 & 5476 & 2334.02 & 1 & 8.37 \\
\hline $\mathrm{R} 209$ & 3 & 916.99 & 536 & 1543.06 & 913.32 & 4591 & 2309.21 & 2 & 13.11 \\
\hline $\mathrm{R} 210$ & 3 & 939.91 & 719 & 1184.84 & 939.37 & 5127 & 7045.56 & 1 & 5.30 \\
\hline $\mathrm{R} 211$ & 2 & 904.14 & 785 & 1670.15 & 904.14 & 4537 & 1670.15 & 53 & 277.88 \\
\hline $\mathrm{RC} 101$ & 14 & 1696.95 & 1293 & 228.75 & 1696.95 & 10052 & 228.75 & 45 & 98.62 \\
\hline RC102 & 12 & 1554.84 & 1154 & 1350.84 & * 1554.75 & 10365 & 4089.89 & 118 & 285.01 \\
\hline RC103 & 11 & 1263.54 & 1634 & 1973.22 & * 1261.67 & 12480 & 8887.18 & 2 & 5.98 \\
\hline RC104 & 10 & 1138.95 & 1533 & 566.86 & * 1135.48 & 12248 & 9584.67 & 5 & 16.44 \\
\hline $\mathrm{RC} 105$ & 14 & 1542.29 & 911 & 1342.22 & 1540.18 & 11671 & 3133.75 & 49 & 121.21 \\
\hline $\mathrm{RC} 105$ & 13 & - & - & - & 1681.56 & 7207 & 5220.97 & 2453 & 5220.97 \\
\hline RC106 & 11 & 1453.59 & 1425 & 1831.30 & * 1424.73 & 10440 & 13621.57 & 514 & 733.54 \\
\hline RC107 & 11 & 1232.26 & 1735 & 821.58 & 1232.26 & 13306 & 821.58 & 8 & 18.08 \\
\hline $\mathrm{RC} 108$ & 10 & 1147.35 & 1681 & 1789.81 & 1140.83 & 12928 & 4134.77 & 11 & 20.06 \\
\hline $\mathrm{RC} 201$ & 4 & 1413.52 & 1061 & 734.08 & 1406.94 & 8366 & 6877.76 & 1 & 2.95 \\
\hline RC202 & 3 & 1378.42 & 899 & 1826.62 & 1376.03 & 7932 & 3001.17 & 23 & 100.63 \\
\hline $\mathrm{RC} 203$ & 3 & 1088.06 & 580 & 410.42 & 1063.68 & 4805 & 2262.31 & 12 & 70.40 \\
\hline $\mathrm{RC} 204$ & 3 & 800.83 & 849 & 662.97 & 799.16 & 7522 & 8742.92 & 1 & 6.31 \\
\hline $\mathrm{RC} 205$ & 4 & 1302.60 & 1066 & 375.94 & 1297.65 & 9141 & 4668.77 & 1 & 2.87 \\
\hline $\mathrm{RC} 206$ & 3 & 1152.03 & 642 & 1798.40 & * 1146.32 & 5459 & 3986.83 & 6 & 36.49 \\
\hline $\mathrm{RC} 207$ & 3 & 1093.74 & 540 & 1528.29 & 1064.40 & 4917 & 4274.48 & 2 & 13.74 \\
\hline $\mathrm{RC} 208$ & 3 & ** 828.14 & 749 & 1522.56 & ** 828.14 & 7232 & 1522.56 & 1 & 4.58 \\
\hline
\end{tabular}

$*$ : a tie with the best known solution; $* *$ : a better solution than the best known solution 
Table 8. The best known values for Solomon's instances according to http://www.sintef.no/static/am/opti/projects/top/vrp/bknown.html (the data was taken on March 6, 2003)

\begin{tabular}{|c|c|c|c|c|c|}
\hline instance & $m$ & distance & instance & $m$ & distance \\
\hline R101 & 19 & 1645.79 & $\mathrm{R} 201$ & 4 & 1252.37 \\
\hline R102 & 17 & 1486.12 & $\mathrm{R} 202$ & 3 & 1191.70 \\
\hline R103 & 13 & 1292.68 & R203 & 3 & 939.54 \\
\hline R104 & 9 & 1007.24 & $\mathrm{R} 204$ & 2 & 825.52 \\
\hline R105 & 14 & 1377.11 & $\mathrm{R} 205$ & 3 & 994.42 \\
\hline R106 & 12 & 1251.98 & $\mathrm{R} 206$ & 3 & 906.14 \\
\hline R107 & 10 & 1104.66 & $\mathrm{R} 207$ & 2 & 893.33 \\
\hline R108 & 9 & 960.88 & $\mathrm{R} 208$ & 2 & 726.75 \\
\hline R109 & 11 & 1194.73 & R209 & 3 & 909.16 \\
\hline R110 & 10 & 1118.59 & $\mathrm{R} 210$ & 3 & 939.34 \\
\hline R111 & 10 & 1096.72 & $\mathrm{R} 211$ & 2 & 892.71 \\
\hline R112 & 9 & 982.14 & & & \\
\hline C101 & 10 & 828.94 & $\mathrm{C} 201$ & 3 & 591.56 \\
\hline C102 & 10 & 828.94 & $\mathrm{C} 202$ & 3 & 591.56 \\
\hline C103 & 10 & 828.06 & $\mathrm{C} 203$ & 3 & 591.17 \\
\hline C104 & 10 & 824.78 & $\mathrm{C} 204$ & 3 & 590.60 \\
\hline $\mathrm{C} 105$ & 10 & 828.94 & $\mathrm{C} 205$ & 3 & 588.88 \\
\hline C106 & 10 & 828.94 & $\mathrm{C} 206$ & 3 & 588.49 \\
\hline C107 & 10 & 828.94 & $\mathrm{C} 207$ & 3 & 588.29 \\
\hline C108 & 10 & 828.94 & $\mathrm{C} 208$ & 3 & 588.32 \\
\hline C109 & 10 & 828.94 & & & \\
\hline $\mathrm{RC} 101$ & 14 & 1696.94 & $\mathrm{RC} 201$ & 4 & 1406.91 \\
\hline $\mathrm{RC} 102$ & 12 & 1554.75 & $\mathrm{RC} 202$ & 3 & 1367.09 \\
\hline $\mathrm{RC} 103$ & 11 & 1261.67 & $\mathrm{RC} 203$ & 3 & 1049.62 \\
\hline $\mathrm{RC} 104$ & 10 & 1135.48 & $\mathrm{RC} 204$ & 3 & 798.41 \\
\hline $\mathrm{RC} 105$ & 13 & 1629.44 & $\mathrm{RC} 205$ & 4 & 1297.19 \\
\hline $\mathrm{RC} 106$ & 11 & 1424.73 & $\mathrm{RC} 206$ & 3 & 1146.32 \\
\hline $\mathrm{RC} 107$ & 11 & 1230.48 & $\mathrm{RC} 207$ & 3 & 1061.14 \\
\hline $\mathrm{RC} 108$ & 10 & 1139.82 & $\mathrm{RC} 208 \dagger$ & 3 & 828.14 \\
\hline
\end{tabular}


Table 9. Benchmark results of various CPUs

\begin{tabular}{lccccc}
\hline CPU & SPECint2000 & SPECint95 & LINPACK & Mflops & Mips \\
\hline Pentium III 1 GHz & $408-442$ & & & & \\
Pentium III 933 MHz & & & $192-234$ & & \\
Pentium III $800 \mathrm{MHz}$ & 344 & 38.0 & & & \\
Sun Ultra $10440 \mathrm{MHz}$ & & 18.1 & $(73)$ & & \\
Pentium $400 \mathrm{MHz}$ & & & $(54)$ & 239 & 940 \\
Pentium 200 MHz & & $5.00-5.47$ & $(24)$ & 107 & 349 \\
Sun Sparc 10 & & & 10 & & \\
\hline
\end{tabular}


Table 10. Estimated running time of various algorithms

\begin{tabular}{|c|c|c|c|c|c|c|}
\hline algorithm & $\mathrm{CPU}$ & $\begin{array}{c}\text { estimated } \\
\text { speed }\end{array}$ & $\begin{array}{l}\text { avg. CPU seconds } \\
\text { for types R \& RC }\end{array}$ & $\begin{array}{l}\text { number } \\
\text { of runs }\end{array}$ & $\begin{array}{l}\text { number } \\
\text { of CPUs }\end{array}$ & $\begin{array}{l}\text { estimated time } \\
\text { on our CPU (s) }\end{array}$ \\
\hline ILS and AMLS & Pentium III $1 \mathrm{GHz}$ & 1 & 2000 or 15000 & 1 & 1 & 2000 or 15000 \\
\hline G\&H (2002) & Pentium $400 \mathrm{MHz}$ & 0.24 & $1088-1420$ & 5 & 4 & $5222-6816$ \\
\hline H\&G (1999) & Pentium $200 \mathrm{MHz}$ & 0.1 & 660-990 & 10 & 1 & 660-990 \\
\hline BBB (2003) & Pentium $400 \mathrm{MHz}$ & 0.24 & 1800 & 3 & 1 & 1300 \\
\hline $\mathrm{Br}(2003)$ & Pentium $200 \mathrm{MHz}$ & 0.1 & $3245-7203$ & 1 & 1 & $325-720$ \\
\hline BVH (2001) & Sun Ultra $10440 \mathrm{MHz}$ & 0.4 & 7200 & 5 & 1 & 14000 \\
\hline
\end{tabular}

